\title{
Supplementary Information for DFT Analysis of Organotin Catalytic Mechanisms in Dehydration Esterification Reactions for Terephthalic Acid and 2,2,4,4--Tetramethyl-1,3-Cyclobutanediol
}

Jennifer A. Clark ${ }^{\dagger}$. Pranav J. Thacker ${ }^{\dagger}$, Charles McGill ${ }^{\dagger}$, Jason R. Miles ${ }^{\dagger}$, Phillip Westmoreland ${ }^{\dagger}$, Kirill Efimenko $^{\dagger}$, Jan Genzer ${ }^{\dagger}$, and Erik E. Santiso ${ }^{*}, \dagger$

${ }^{\dagger}$ Department of Chemical and Biomolecular Engineering, North Carolina State University, Raleigh, NC, 27695, USA

\section{S1: Breaking Resonance with Terephthalic Acid}

In the main body of this work, we suggested that reactions involving terephthalic ligands have high activation energies. We reason that the diacid with a phenyl group creates a large conjugated moiety that delocalizes the electron density withdrawn from the tin center. Before breaking the resonance and subsequently removing the acid as a ligand, that density would need to return to the catalyst. As a test of this supposition, we scanned the dihedral angle between the Sn-O bond connecting the acid, and the phenyl plain, $\theta_{\text {O,phenyl. }}$ We varied the dihedral in $5^{\circ}$ increments, keeping all other adjacent dihedrals and angles frozen. Notice in Figure S1 that as the dihedral changes, the free energy increases, and the catalyst's partial charges adjust.

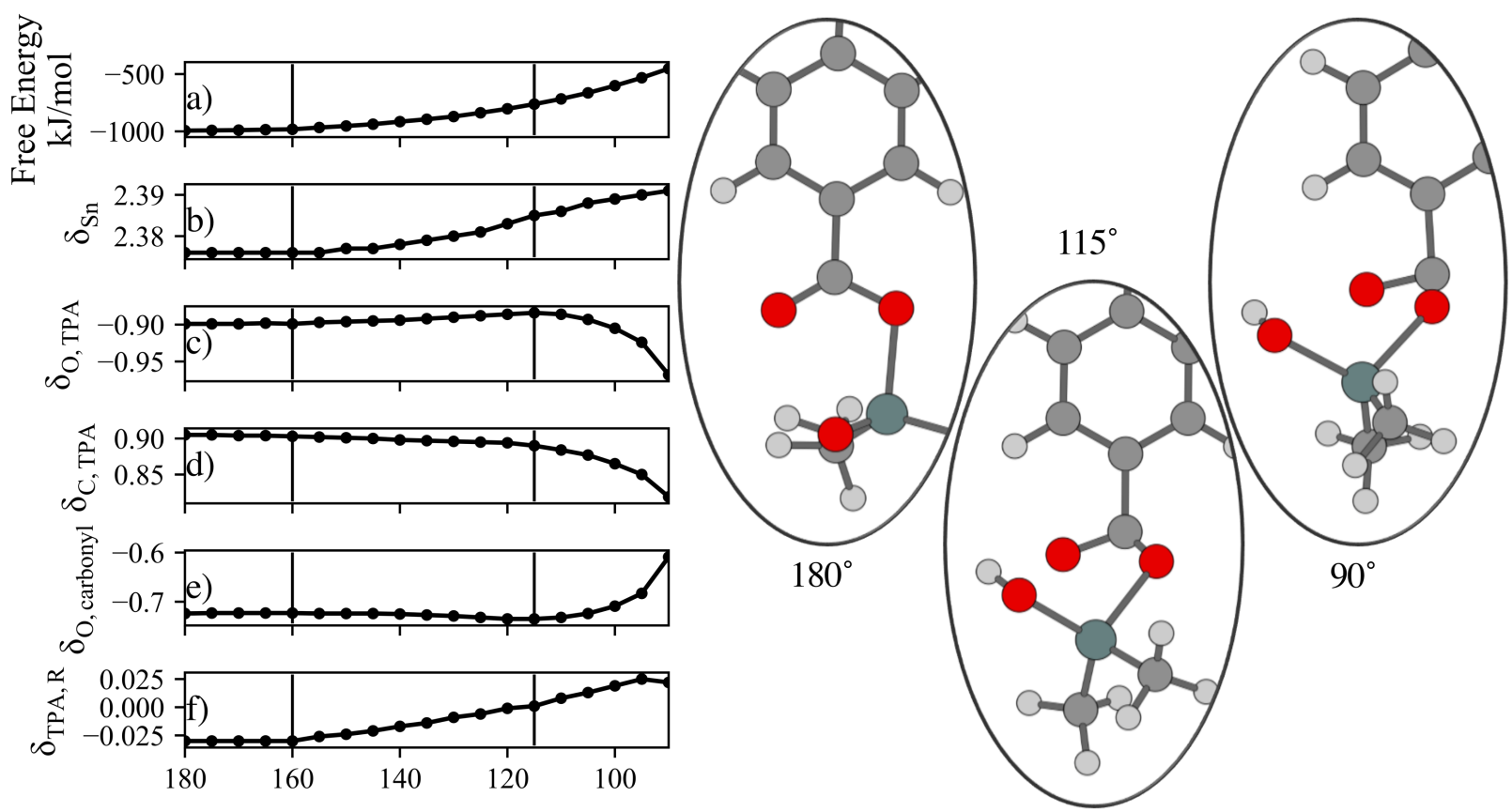

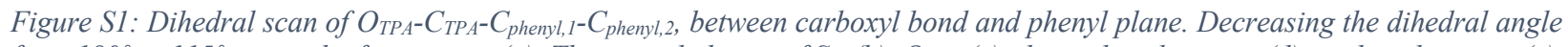
from $180^{\circ}$ to $115^{\circ}$ raises the free energy (a). The partial charge of $S n(b), O_{T P A}(c)$, the carboxyl oxygen (d), carbonyl oxygen (e), and remaining structure (i.e., phenyl group and opposing carboxyl group) (f) vary in response.

TPA as a ligand delocalizes electron density from the tin center. Before reducing the catalytic center, the hydroxyl oxygen that binds to the tin has a partial charge of $\delta_{\mathrm{O}, \mathrm{TPA}}=-0.753 e$, the carboxyl carbon, $\mathrm{C}_{\mathrm{TPA}}$ has a partial charge of $0.905 e$, the carbonyl oxygen has a charge of $-0.677 e$, and the combination of the phenyl and opposing carboxyl group has charge of $\delta_{\mathrm{TPA}, \mathrm{R}}=-0.015 \mathrm{e}$. Once bound to the catalytic center, these values increase in magnitude to $-0.899 e, 0.905 e,-0.724 e$, and $-0.030 e$, respectively, when the dihedral angle $\theta_{\text {O,phenyl }}=180^{\circ}$. Increase in electron density around these atoms indicates electron delocalization from the tin complex to the TPA ligand structure, including the carboxylate and subtly into the aromatic group. 
Figure S1 shows that $\theta_{\mathrm{O} \text {,phenyl }}$ may decrease by up to $20^{\circ}$ before changes in the partial charges occur. After this point, Sn, OTPA and the remainingTPA ligand start losing electron density while $\mathrm{O}_{\text {carbonyl }}$ and $\mathrm{C}_{\mathrm{TPA}}$ gain it. These trends continue until $\mathrm{O}_{\text {TPA }}$ reaches a minimum negative charge at $\theta_{\text {O,phenyl }}=115^{\circ}$. At this point the $\mathrm{C}-\mathrm{O}$ bond connecting a TPA ligand to the metal begins to break. The charges $\delta_{\mathrm{O}, \text { TPA }}$ and $\delta_{\mathrm{C}, \text { TPA }}$ both change at an increasing rate, while $\mathrm{O}_{\text {carbonyl }}$ begins to donate rather than withdraw. From this work we find that the carboxyl dihedral angle can fluctuate by $20^{\circ}$ with little consequence and up to $65^{\circ}$ with a strained but supported bond. Once the dihedral angle goes beyond $65^{\circ}$ the bond begins to deteriorate although it remains rich in density from the catalyst.

\section{S2: Scan TPA Reduction of Tin Oxide}

The diacid, TPA, reduces dimethyltin oxide in a barrierless manner as shown in Figure S2. To confirm that no transition state exists, a scan of the atomic distance between the hydrogen atom and the oxide, although we have included the atomic distance with both oxygen atoms in Table S1.

\begin{tabular}{|l|c|c|c|c|c|}
\hline \multicolumn{6}{|c|}{ Table S1: Atomic distance between reacting hydrogen atom and participating oxygen atoms } \\
\hline \multicolumn{1}{|c|}{ Oxygen } & Isolated & Point 1 & Point 2 & Point 3 & Point 4 \\
\hline TPA & 0.9703 & 1.0435 & 1.8269 & NA & NA \\
Catalyst & NA & 1.5944 & 1.0614 & 1.0604 & 0.9544 \\
\hline
\end{tabular}

If this reaction has a barrier we would expect this scan to increase in energy as the transition state is approached, followed by a decrease in energy. Instead, as the reacting hydrogen atom becomes distant from TPA and interacts with the catalytic oxide, the energy steadily decreases. This trend continues until the association between the newly formed hydroxide ligand and the TPA ligand carbonyl group releases. At this point, the relaxation of the ligands away from each other is lower in energy than maintaining the association. This leads to a noticeable shift in positioning and drop in energy between points 2 and 3 below with only a change of $0.001 \AA$ in the scanned bond length.
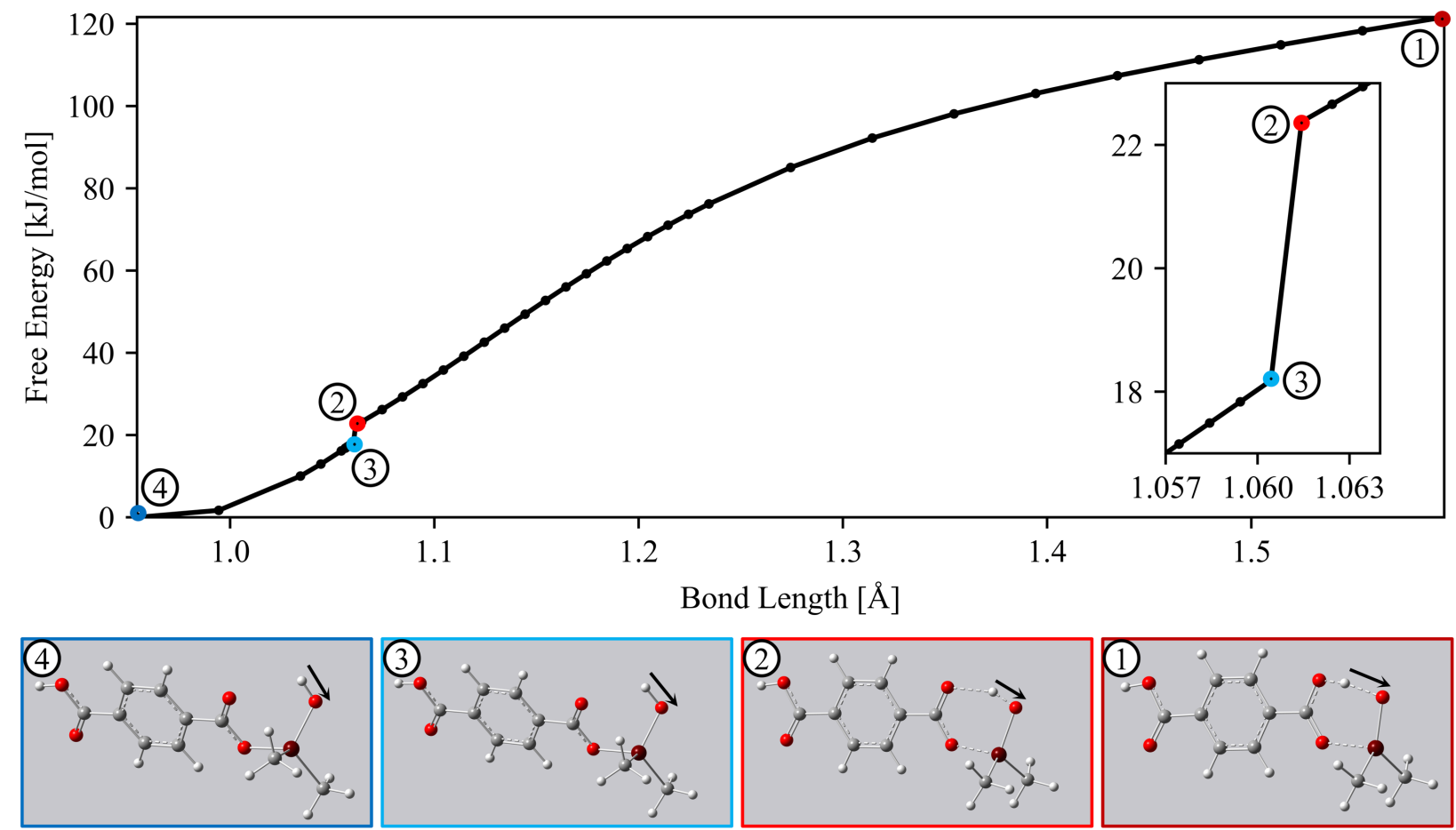

Figure S2: Scan of hydrogen - oxide bond as TPA reduces tin complex. The black arrows indicate the direction of hydrogen motion in the course of the scan, as it decreases in atomic distance with oxygen. 


\section{S3: Coordinated Intermediates}

In the course of a reaction, reactants may assemble into a complex before a reaction, however the electronic structure calculations may show this step is unfavorable. Electronic structure calculations often use implicit solvent: explicit molecules will then interact to minimize the free energy well. Before taking this structure as representative of the real system, the associated free energy should be compared to the sum of the energies associated with the individual structures. A lower free energy indicates favorable complexation while a higher free energy arises as an artifact of the energy minimization in implicit solvent. This loose complex then contains low vibrational modes that poorly represent the thermodynamics of the system. These low vibrational modes restrict the system to decrease its entropy, thus increasing the free energy. In Table S2 below, the free energy of the coordinated structure is compared to the sum of the free energies of individual reactants. The lower energy is highlighted in green as the correct precursor to the transition state. Note that for neutral reactions, complexation of the reactants does not prove to be the free energy minimum. Thus, throughout the main body of this work we calculated the activation energy at various stages using the sum of the individual free energies of reactants. However, reactions involving hydronium are shown to produce significant complexes and so are included in the free energy coordinates of this work.

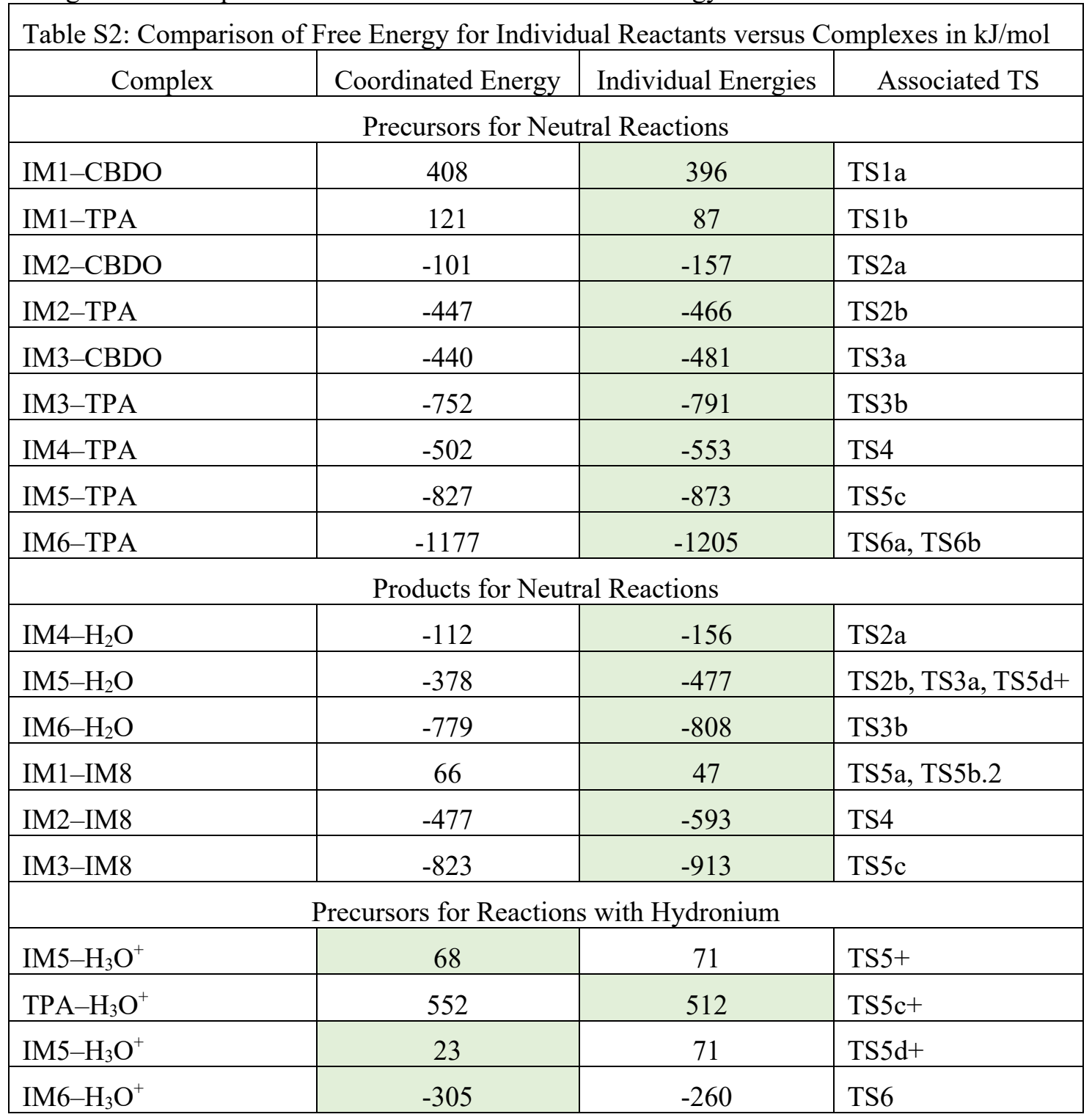




\begin{tabular}{|l|c|c|l|}
\hline \multicolumn{4}{|c|}{ Products for Reactions with Hydronium } \\
\hline IM8+ & 417 & 472 & TS5d+, TS6 \\
\hline IM1- $\mathrm{H}_{3} \mathrm{O}^{+}$ & 846 & 1031 & TS5+ \\
\hline $\mathrm{IM} 1+-\mathrm{IM} 8$ & 104 & 84 & TS5+ \\
\hline $\mathrm{IM} 3-\mathrm{H}_{3} \mathrm{O}^{+}$ & 69 & 154 & TS5c + \\
\hline
\end{tabular}

\section{S4: Bond Lengths of Intramolecular Acyl Transfer Reactions}

To accompany Figure 6 of the main text, we list the relevant bonds lengths for each step in the reaction coordinate. These value are summarized in Table S3.

Table S3: Bonds lengths of optimized structures in intramolecular acyl transfer reactions $(\AA)$

\begin{tabular}{|c|c|c|c|c|c|c|c|}
\hline & $\mathrm{Sn}-\mathrm{Me}$ & $\mathrm{Sn}-\mathrm{O}_{\mathrm{CBDO}}$ & $\mathrm{Sn}-\mathrm{O}_{\mathrm{TPA}}$ & Sn-O $\mathrm{O}_{\text {carbonyl }}$ & $\mathrm{C}_{\mathrm{TPA}}-\mathrm{O}_{\mathrm{CBDO}}$ & $\mathrm{C}_{\text {TPA }}-\mathrm{O}_{\mathrm{TPA}}$ & $\mathrm{C}_{\mathrm{TPA}}-\mathrm{O}_{\text {carbonyl }}$ \\
\hline \multicolumn{8}{|c|}{ Reactants } \\
\hline IM5 & 2.106 & 1.922 & 2.010 & - & - & 1.337 & 1.252 \\
\hline IM5+ & 2.100 & 1.903 & 2.240 & - & - & 1.387 & 1.205 \\
\hline \multicolumn{8}{|c|}{ Box Transfer } \\
\hline TS5a & 2.122 & 2.212 & 1.860 & - & 1.466 & 2.096 & 1.209 \\
\hline \multicolumn{8}{|c|}{ Coordinated Transfer } \\
\hline TS5b.1 & 2.125 & 2.308 & 2.053 & 2.149 & 1.763 & 1.349 & 1.318 \\
\hline IM7 & 2.117 & - & 1.990 & 1.986 & 1.414 & 1.405 & 1.403 \\
\hline TS5b.2 & 2.125 & - & 1.869 & 2.132 & 1.310 & 2.211 & 1.281 \\
\hline \multicolumn{8}{|c|}{ Box + Transfer } \\
\hline TS5+ & 2.096 & 2.060 & 2.005 & - & 1.627 & 1.723 & 1.213 \\
\hline $\mathrm{IM} 1+$ & 2.098 & - & 1.882 & - & - & - & - \\
\hline \multicolumn{8}{|c|}{ Products } \\
\hline IM1 & 2.128 & - & 1.817 & - & - & - & - \\
\hline IM8 & - & - & - & - & 1.335 & - & 1.218 \\
\hline
\end{tabular}

For clarity, the bond lengths shown in Table S3 can be visualized in Figure S3, where the geometries for each structure represented are shown. 

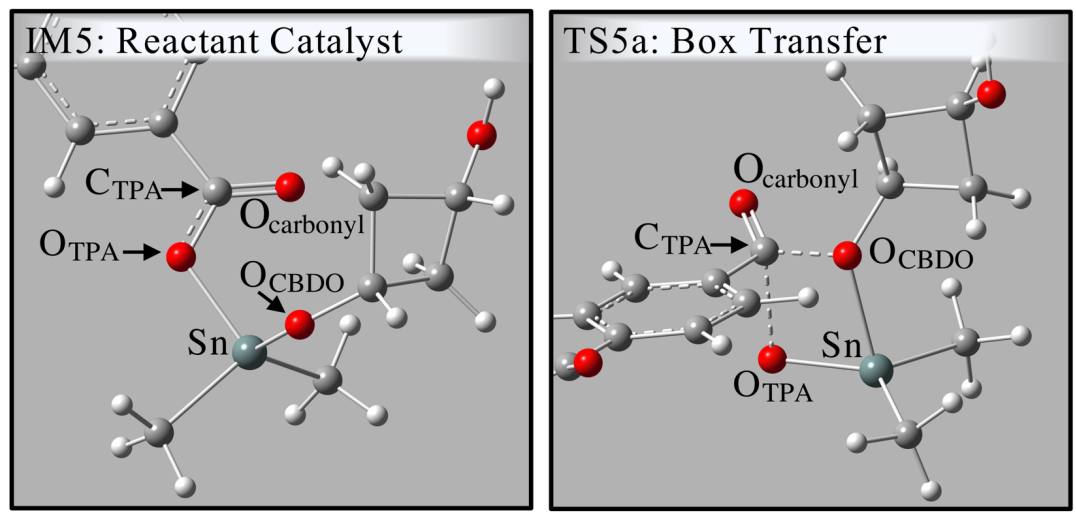

IM1: Original Catalyst
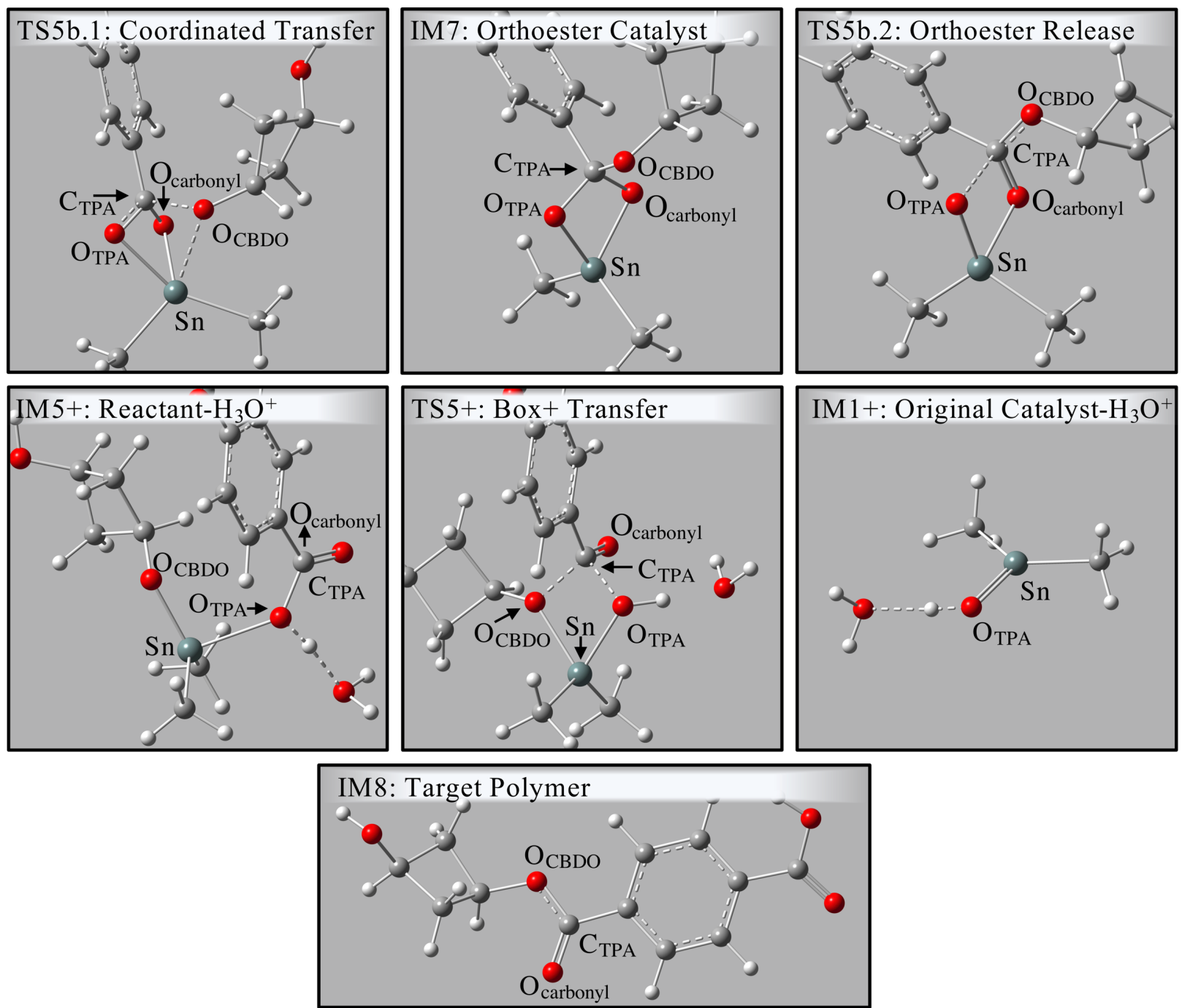

Figure S3: Stationary points and transition states represented in Figure 6 of the main text. The atom labels are provided to clarify the bond lengths in Table S3. 


\section{S5: Intermolecular Esterification}

In addition to intramolecular reactions, we also investigated intermolecular pathways and show two more mechanisms. We term the intermolecular acyl transfer with an incoming acid, acid transfer, as it exchanges its hydroxyl group with an alkoxy ligand to reproduce IM2, as shown in Figure S4a. We refer to the intermolecular acyl transfer case, where alcohol exchanges with the catalyst, as alcohol transfer. In this reaction, a hydroxyl group remains, this time to regenerate IM3 (Figure S4b). In this treatment, each intramolecular reaction (box and coordinated transfers) requires both monomer to be ligands on the metal center. The alcohol and acid transfers require one carboxylate or one alkoxy ligand, respectively. We obtained the transition states for alcohol transfer with the catalytic complexes IM5 and IM6, and the acid transfer reactions of IM4 and IM5. However, in our comparison of alcohol and acid transfers, we will use the common starting structure, IM5.

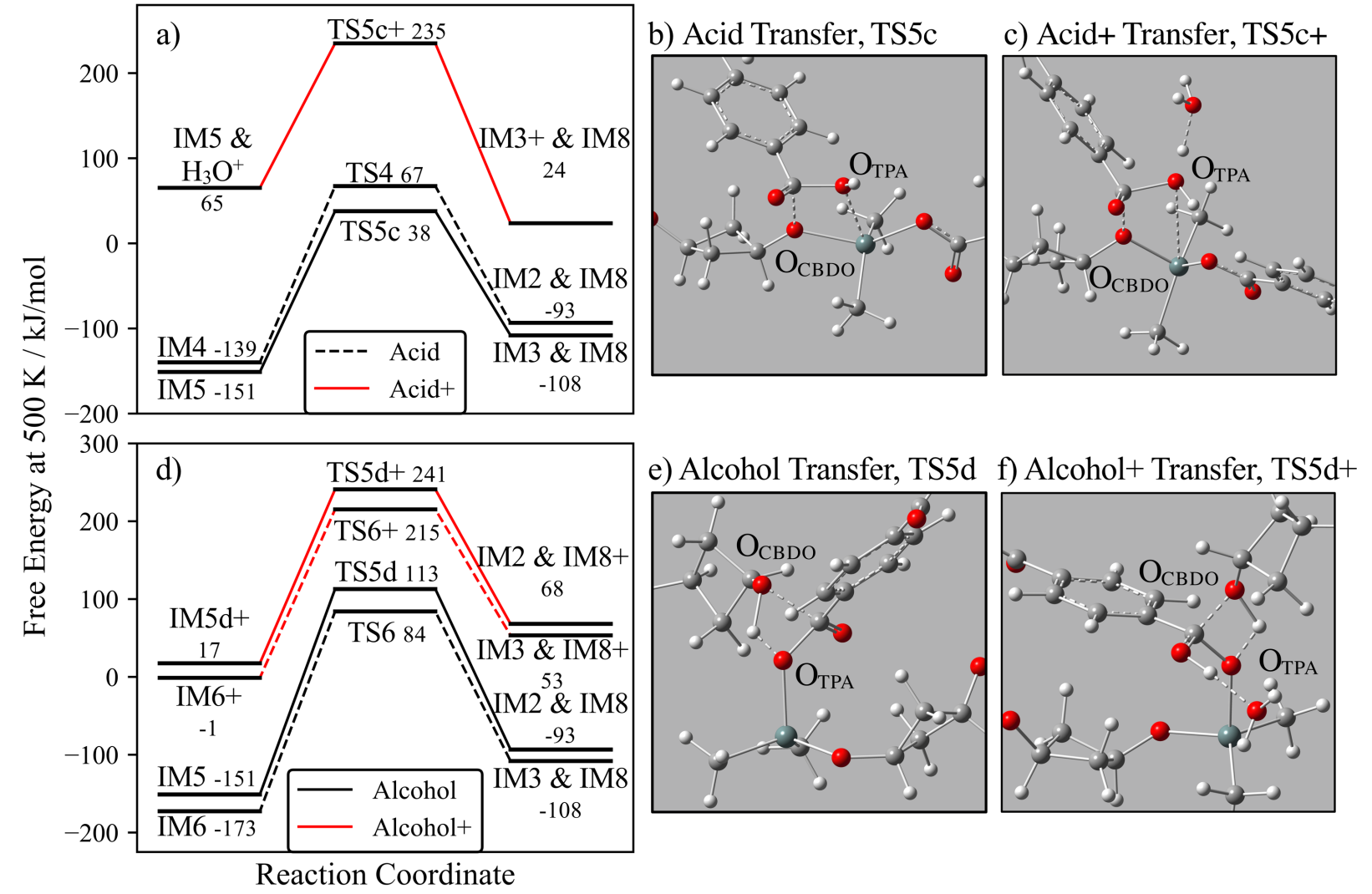

Figure S4: We identified two possible intramolecular transition states that would result in the esterification. (a) The first type of intermolecular reaction is an acyl transfer, where the hydroxyl group on the acid exchanges with the alkoxy ligand. (b) A simulation depiction shows the acid transfer mechanism of IM5. (c) When a hydronium molecule coordinates to $O_{T P A}$, the acid acyl transfer takes place with a different geometry. (d) The second type of intermolecular option allows a nucleophilic monomer to coordinate to the carboxylate ligand and then undergo a proton transfer to complete the acyl substitution. (e) The alcohol transfer mechanism consists of the concerted transfer of a proton and the detachment of the resulting hydroxyl group. (f) A hydronium coordinated to the TPA carbonyl oxygen aids in the alcohol acyl transfer.

The first intermolecular acyl transfer (intermolecular acid acyl transfer) has the lowest activation barrier of $189 \mathrm{~kJ} / \mathrm{mol}$ for its neutral form. This mechanism has the advantage that it does not involve a carboxylate ligand. A TPA monomer reacts with a CBDO ligand to produce IM3 and the polymer, IM8. Although the carboxylate breaks its conjugation with the hydroxyl group, it bonds with an alkoxy at a dihedral angle of $112^{\circ}$ in TS5c, as shown in Figure S4b. Not only does this acyl transfer not have the penalty of removing resonance stability with TPA, but in the course of the reaction, it does not require additional electron density from the bystander ligands. We observed similar behavior in dehydrating a hydoxylated tin complex with TPA. In TS5c, although close, the partial charges $\delta_{\mathrm{O}, \mathrm{TPA}}=-0.890 e$ and $\delta_{\mathrm{O}, \mathrm{CBDO}}=-0.895 e$ indicate that the 
hydroxyl $\mathrm{O}_{\text {TPA }}$ is the appropriate leaving group. This transition state then is the first where $\mathrm{O}_{\mathrm{CBDO}}$ has more electron density than $\mathrm{O}_{\mathrm{TPA}}$.

The second intermolecular acyl transfer option, alcohol transfer, has the advantage that it does not require excessive torsion in TPA to get to the transition state. However, the electron density must be redirected to the approaching alcohol to form a conjugated system. Just as the box-transfer mechanism suffered a large energy penalty in relinquishing catalyst electron density that the carboxylate system withdrew, so does the alcohol transfer. However, because this is an intermolecular acyl transfer, a bystander CBDO ligand remains. Thus, one less bond on the tin is broken. Instead of regenerating the oxide catalyst, this alcoholysis reproduces IM2. During the transition state, TS5d, $\mathrm{O}_{\text {TPA }}$ pulls another $0.019 e$ from tin and $0.025 e$ from the methyl ligands. The bystander CBDO contributes just $0.008 e$ to the reaction site. To secure the proton from an approaching $\mathrm{CBDO}$ alcohol, as shown in Figure S4e, $\mathrm{O}_{\text {TPA }}$ retracted -0.057 e from the carboxylate ligand. This transition state results in less complex electron movement, as the catalyst is breaking only one bond with the metal center. However, $\mathrm{O}_{\text {TPA }}$ has a larger partial charge of $-0.992 e$, while the competing $\mathrm{O}_{\mathrm{CBDO}}$ has a lower charge of $-0.784 e$. Thus, removing the TPA ligand requires high activation energy of $264 \mathrm{~kJ} / \mathrm{mol}$ to overcome this large difference in charges.

\begin{tabular}{|c|c|c|c|c|c|c|c|c|c|}
\hline & $\mathrm{Sn}$ & $\begin{array}{l}\text { Methyl } \\
\text { Groups }\end{array}$ & $\begin{array}{c}\text { Bystander } \\
\text { Ligand }\end{array}$ & $\mathrm{O}_{\text {TPA }}$ & $\mathrm{C}_{\mathrm{TPA}}$ & $\begin{array}{c}\text { Remaining } \\
\text { TPA }\end{array}$ & $\mathrm{H}_{\text {monomer }}$ & $\mathrm{O}_{\mathrm{CBDO}}$ & $\begin{array}{c}\text { Remaining } \\
\text { CBDO }\end{array}$ \\
\hline \multicolumn{3}{|c|}{ TS4: $E_{a}=207 \mathrm{~kJ} / \mathrm{mol}$} & $\underline{\mathrm{CBDO}}$ & \multicolumn{3}{|c|}{ Monomer } & & \multicolumn{2}{|l|}{$\underline{\text { Ligand }}$} \\
\hline React & 2.405 & -0.975 & -0.728 & -0.736 & 0.904 & -0.694 & 0.528 & -1.015 & 0.312 \\
\hline TS & 2.448 & -0.976 & -0.727 & -0.895 & 0.938 & -0.884 & 0.551 & -0.837 & 0.382 \\
\hline$\Delta \delta$ & 0.043 & -0.001 & 0.001 & -0.159 & 0.034 & -0.190 & 0.023 & 0.178 & 0.070 \\
\hline \multicolumn{3}{|c|}{ TS5c: $E_{a}=189 \mathrm{~kJ} / \mathrm{mol}$} & $\underline{\mathrm{TPA}}$ & \multicolumn{3}{|c|}{ Monomer } & & \multicolumn{2}{|l|}{$\underline{\text { Ligand }}$} \\
\hline React & 2.392 & -0.938 & -0.754 & -0.736 & 0.904 & -0.694 & 0.528 & -1.020 & 0.322 \\
\hline TS & 2.426 & -0.937 & -0.753 & -0.890 & 0.941 & -0.810 & 0.557 & -0.895 & 0.363 \\
\hline$\Delta \delta$ & 0.034 & 0.001 & 0.001 & -0.154 & 0.037 & -0.116 & 0.029 & 0.125 & 0.041 \\
\hline \multicolumn{3}{|c|}{ TS5d: $E_{a}=264 \mathrm{~kJ} / \mathrm{mol}$} & $\underline{\mathrm{CBDO}}$ & \multicolumn{3}{|l|}{ Ligand } & & \multicolumn{2}{|c|}{ Monomer } \\
\hline React & 2.392 & -0.938 & -0.698 & -0.912 & 0.910 & -0.752 & 0.491 & -0.804 & 0.313 \\
\hline $\mathrm{TS}$ & 2.411 & -0.913 & -0.690 & -0.992 & 0.918 & -0.817 & 0.547 & -0.784 & 0.321 \\
\hline$\Delta \delta$ & 0.019 & 0.025 & 0.008 & -0.080 & 0.008 & -0.065 & -0.056 & 0.020 & 0.008 \\
\hline \multicolumn{3}{|c|}{ TS6: $E_{a}=257 \mathrm{~kJ} / \mathrm{mol}$} & $\underline{\text { TPA }}$ & \multicolumn{3}{|l|}{ Ligand } & & \multicolumn{2}{|c|}{ Monomer } \\
\hline React & 2.369 & -0.915 & -0.690 & -0.919 & 0.907 & -0.751 & 0.491 & -0.804 & 0.313 \\
\hline $\mathrm{TS}$ & 2.383 & -0.889 & -0.671 & -0.984 & 0.917 & -0.800 & 0.549 & -0.809 & 0.306 \\
\hline$\Delta \delta$ & 0.014 & 0.026 & 0.019 & -0.065 & 0.010 & -0.049 & 0.058 & -0.005 & -0.007 \\
\hline \multicolumn{3}{|c|}{$\underline{\mathbf{T S 5 c}+:} E_{a}=170 \mathrm{~kJ} / \mathrm{mol}$} & TPA & \multicolumn{2}{|c|}{$\underline{\text { Monomer }}$} & & & \multicolumn{2}{|l|}{ Ligand } \\
\hline React & 2.392 & -0.938 & -0.754 & -0.778 & 0.912 & -0.598 & 0.558 & -1.020 & 0.322 \\
\hline $\mathrm{TS}$ & 2.408 & -0.890 & -0.669 & -0.815 & 0.935 & -0.657 & 0.556 & -0.910 & 0.391 \\
\hline$\Delta \delta$ & 0.016 & 0.048 & 0.085 & -0.037 & 0.023 & -0.059 & -0.002 & 0.110 & 0.069 \\
\hline
\end{tabular}




\begin{tabular}{|c|c|c|c|c|c|c|c|c|c|}
\hline \multicolumn{3}{|c|}{ TS5d+: $E_{a}=224 \mathrm{~kJ} / \mathrm{mol}$} & \multirow{2}{*}{$\frac{\mathrm{CBDO}}{-0.689}$} & \multicolumn{3}{|l|}{ Ligand } & \multirow[b]{2}{*}{0.491} & \multicolumn{2}{|c|}{ Monomer } \\
\hline React & 2.388 & -0.875 & & -0.795 & 0.984 & -0.647 & & -0.804 & 0.313 \\
\hline TS & 2.425 & -0.892 & -0.686 & -0.983 & 0.990 & -0.781 & 0.575 & -0.673 & 0.432 \\
\hline $\mathrm{D} \delta$ & 0.037 & -0.017 & 0.003 & -0.188 & 0.006 & -0.134 & 0.084 & 0.131 & 0.119 \\
\hline \multicolumn{3}{|c|}{$\underline{\mathbf{T S 6}}+: E_{a}=216 \mathrm{~kJ} / \mathrm{mol}$} & $\underline{\text { TPA }}$ & Ligand & & & & Monom & \\
\hline React & 2.357 & -0.861 & -0.656 & -0.790 & 0.979 & -0.656 & 0.491 & -0.804 & 0.313 \\
\hline TS & 2.389 & -0.869 & -0.668 & -0.984 & 0.990 & -0.779 & 0.569 & -0.675 & 0.428 \\
\hline$\Delta \delta$ & 0.032 & -0.008 & -0.012 & -0.194 & 0.011 & -0.123 & 0.078 & 0.129 & 0.115 \\
\hline
\end{tabular}

Recall that if two ligands of the same monomer are on the metal center, intramolecular acyl transfer is impossible, leaving only the acid and alcohol transfer options for IM4 and IM6, respectively. These intermolecular options reproduce four-coordinate catalyst structures, IM2 for acid transfer with IM4, and IM3 for alcohol transfer with IM6. These catalysts' free energies with hydroxyl ligands indicate they are more stable than the original tin oxide. We are interested to note the changes in activation energy when the alternative monomer is used as a bystander ligand, making the comparison using Figure S4.

For the alcohol transfer reactions, TS5d and TS6, the resulting activation energies are 264 and $257 \mathrm{~kJ} / \mathrm{mol}$, respectively. The difference between these transition states is that TS5d uses an alkoxy bystander ligand while TS6 involves a carboxyl ligand. The presence of two TPA ligands in IM6 lowers the negative partial charge on $\mathrm{O}_{\text {TPA }}$, as they are more electron-withdrawing than the alkoxy alternative. In IM5, $\delta_{\text {Lig,TPA }}$ is $0.754 e$, while in IM6 $\delta_{\text {Lig,TPA }}$ becomes $-0.690 e$. Despite the increased electronegativity of a TPA bystander, the conjugated nature of this system increases the electron density available, as is shown in several ways. For one, the joint partial charge of the metal and bystander ligands (methyl and monomer) has a $0.008 e$ larger positive charge for the precursor IM6 while it is $0.015 e$ larger for TS6. With more electron density available, less of the density of the incoming monomer must be accessible to ensure a reaction, lowering the activation energy. This is demonstrated with the changes in partial charge of $\mathrm{O}_{\mathrm{CBDO}}$. During TS6, only $0.005 e$ is withdrawn from $\mathrm{O}_{\mathrm{CBDO}}$, while in $\mathrm{TS} 5 \mathrm{~d}, \delta_{\mathrm{O}, \mathrm{CBDO}}$ decreases by $0.02 e$. This could correlate with the additional $0.016 e$ withdrawn from the phenyl group of the TPA ligand. The more electron-withdrawing TPA and its resonance-stabilizing character as a bystander ligand, lower the activation barrier of alcohol acyl transfer.

In comparing the acid transfer of IM4 with that of IM5, the respective activation energies are 207 and 189 $\mathrm{kJ} / \mathrm{mol}$. When two alkoxy ligands are present on IM4, there is a greater positive charge on the tin, $\delta_{\mathrm{Sn}, \mathrm{IM} 4}=2.405 e$, when compared to IM5 where $\delta_{\mathrm{Sn}, \mathrm{IM} 5}=2.392 e$. The unconjugated electron withdrawal of the alkoxy ligands results in polarized bonds with the tin, which in turn polarizes the bonds between tin and the inert methyl ligands. The methyl groups then increase their electron withdrawal by a combined total of $0.037 e$. With the much more polar environment around the tin center, less electron density is available to coordinate with $\mathrm{O}_{\text {TPA. }}$. To make up this difference in the transition state, tin's positive charge raises by $0.043 e$ and the reacting alcohol ligand loses $0.077 e$ more in electron density in TS4 than TS5c. All this is funneled into the incoming TPA monomer to increase $\mathrm{O}_{\text {TPA }}$ to the needed partial charge. With so much more redistribution of electron density, a higher activation energy is required.

The addition of hydronium as a model proton donor facilitates both the alcohol and acid transfers by increasing the partial positive charge of the reactive carboxylate carbon. The changes in partial charges for these reactions are shown in Table S4. Although free energy values are calculated using stable individual species for TS5c+, in Table S4 we reported the reactant partial charges of coordinated species, to facilitate understanding of the environment leading up to the transition state. All other structures in Figure S4 are the coordinated strcutures listed. In the alcohol transfer reaction, the effect of a bystander CBDO or TPA remains the same when hydronium coordinates to the carboxyl oxygen. However, the reacting TPA ligand 
withdraws more electron density from the catalyst. With this positive charge on the carboxylate, the central carbon increased its negative charge by $0.074 e$ for IM5+ and $0.072 e$ from IM6+, thus increasing its attraction to a nucleophilic CBDO hydroxyl group. This electron withdrawal also serves to improve $\mathrm{O}_{\text {TPA }}$ as a leaving group as its partial charge in IM5+ and IM6+ are now lower than $\mathrm{O}_{\mathrm{CBDO}}$. However, like the intramolecular acyl transfer, the amount of electron density withdrawn from the complex continues to lead to higher energy barriers in reacting.

This same sort of improvement in key reactive sites is shown in the acid transfer TS5c+. The carboxylate center, $\mathrm{C}_{\mathrm{TPA}}$ experiences a 0.007 increase in partial positive charge. This mechanism is different from the previous one in that we found a transition state for hydronium coordinated to $\mathrm{O}_{\text {TPA. }}$. Unlike the neutral form of this reaction, $\mathrm{O}_{\text {TPA }}$ is not the obvious leaving group when comparing partial charges. However, the positively charged hydronium pulls at the electron density of $\mathrm{O}_{\text {TPA }}$, facilitating an electron-hopping between $\mathrm{O}_{\mathrm{CBDO}}, \mathrm{C}_{\mathrm{TPA}}, \mathrm{O}_{\mathrm{TPA}}$, and $\mathrm{Sn}$. We were not able to identify any TS4+, presumably because IM4 lacks a conjugated system. With a more electron-deficient $\mathrm{C}_{\mathrm{TPA}}$, the burden is on $\mathrm{O}_{\mathrm{CBDO}}$ to delocalize electron density from the catalyst.

Here we find that a metal-organic catalyst may complement this process by acting as a source of electron density. However, each of these proposed reactions has a prohibitively high activation energy, although understanding their ranking has provided useful insight.

\section{S6: Box and Coordinated Transfer Mechanisms for Post-Transition Metals and Titanium}

When exploring the intramolecular acyl transfer options in this work, we distinguished two mechanisms. The box acyl transfer entails the acid twisting out of plane with its carboxylate as the nucleophilic oxygen of the CBDO alkoxy ligand coordinates to the carboxylate carbon. We refer to this mechanism as a box transfer mechanism because of the geometry. This new ester then releases oxygen from the carboxylate to form tin oxide. We also identified a mechanism where both carboxylate oxygens coordinate to the tin center and the alkoxy ligand hops from the metal center to the electrophilic carboxylic carbon, forming an ortho-ester. We termed this coordinated transfer. We tested these two mechanisms for all post-transition metals plus Ti(IV).

The activation energy of this mechanism for all post-transition metals is shown in Figure S5. In addition to the two monomer ligands, each metal had enough methyl ligands to satisfy their respective oxidation states. The metals with the lowest box

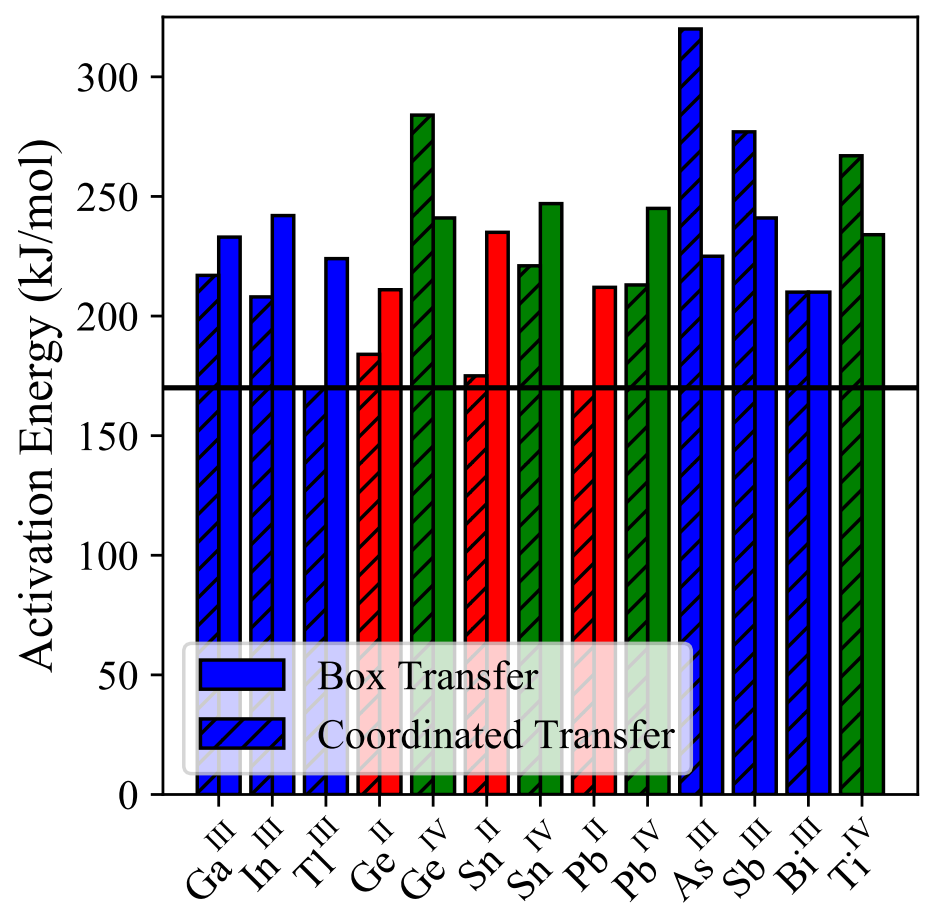

Figure S5: Activation energies of intramolecular acyl transfers for post transition metals, both box acyl transfer and coordinated transfer. Oxidation sates of 2, 3, and 4 are colored red, blue, and green respectively. transfer activation energy rank as $\mathrm{Bi}(\mathrm{III})<\mathrm{Ge}$ (II) $<\mathrm{Pb}$ (II) with similar activation energies of $\sim 210 \mathrm{~kJ} / \mathrm{mol}$. Our metal of interest in this work, $\mathrm{Sn}(\mathrm{IV})$, has the highest activation energy compared to other metals. The alternative intramolecular acyl transfer, coordinated transfer, is more favorable. The ranking of lowest activation energy for the coordinated transfer becomes $\mathrm{Pb}(\mathrm{II})<\mathrm{Tl}(\mathrm{III})<\mathrm{Sn}(\mathrm{II})<\mathrm{Ge}(\mathrm{II})$. These metals are all an improvement, yielding lower 
activation energies than the lowest box transfer barrier for Bi(III). Here $\mathrm{Sn}(\mathrm{IV})$ ranks ninth, with a $51 \mathrm{~kJ} / \mathrm{mol}$ gap from Pb's lowest activation barrier (II).

\section{S7: Box and Coordinated Transfer Mechanisms for Tin with various Ligands}

Our choice of methyl ligands in the main text of this work is due to the historical use of dimethyltin oxide. ${ }^{1}$ However, alternative functional group types and alkane chain length would affect the reaction rate of esterification. For both intramolecular acyl transfers, whether box or coordinated, we tested several functional group types: alkyl, alkoxy, carboxy, or a combination of one alkyl and one alkoxy group (known as a stannone). Figure S6 shows the results of this study, where the electron-donating functional groups contain either one, two, or four carbon atoms.

a)

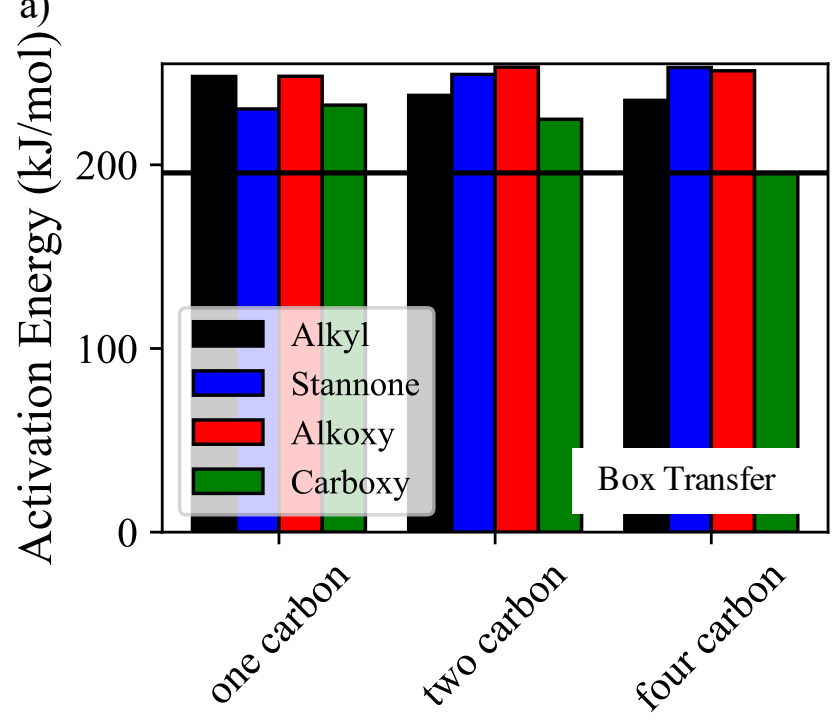

b)

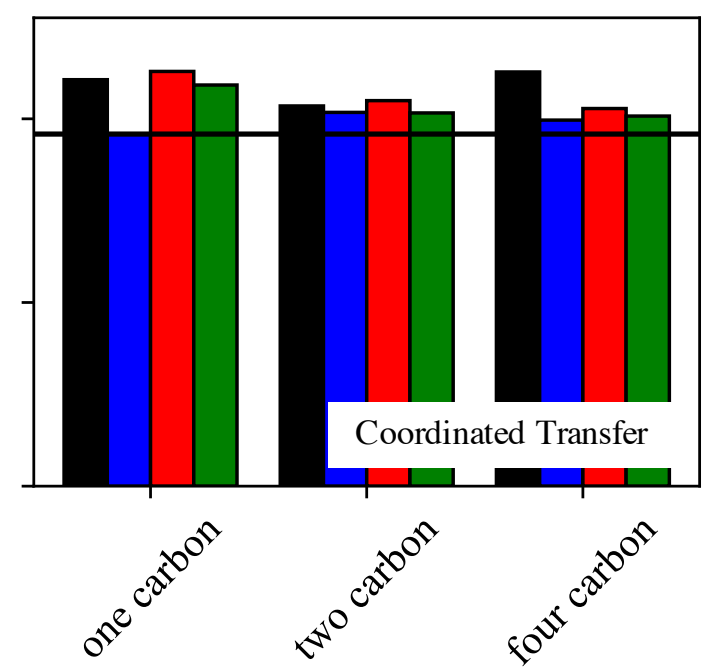

Figure S6: Intramolecular acyl transfer activation energies. Various functional group types, either alkyl, alkoxy, carboxy, or a stannone (one alkyl and one alkoxy) were tested. Either one, two, or four carbon atoms within each functional group type were evaluated for (a) box and (b) coordinated transfer.

For the box transfer mechanism in Figure S6a, it is apparent that chain length plays a significant role. For both alkyl and carboxyl bystander ligands, a longer carbon chain leads to a smaller activation energy. When an alkoxy ligand is present on the metal we observe the opposite trend. This trend seems to result from an increased positive charge of the tin, as shown in Figure S7a. Methyl groups are easily polarizable and so do not withdraw electron density. A sharp notable change is expected when comparing ligands with one and two carbon atoms. In carboxy ligands, we see that formate and acetate are more similar in behavior than butyrate. This is due to our decision to include the carbonyl carbon in our count so that the acetate methyl group is still polarizable. However, we found in the body of this work that the alkoxy group CBDO causes an increase in the activation energy, despite the lower charge of tin. Here we see that this is true in the presence of any alkoxy group, where the charge on tin is notably lower than the alternatives, yet the activation energy remains high. The alkyl and carboxy ligands are weaker electrophiles than TPA, while all the alkoxy ligands are more electrophilic. Thus, TPA is not able to withdraw as much electron density, as seen in Figure S7b and S7c, causing a higher activation barrier. 


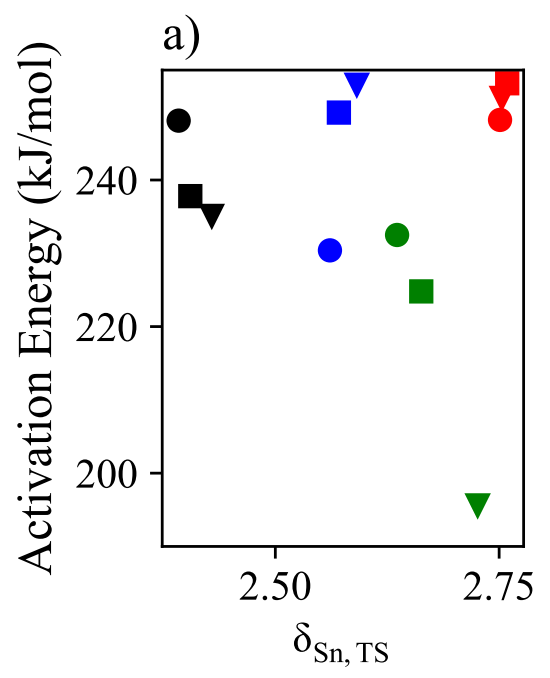

b)

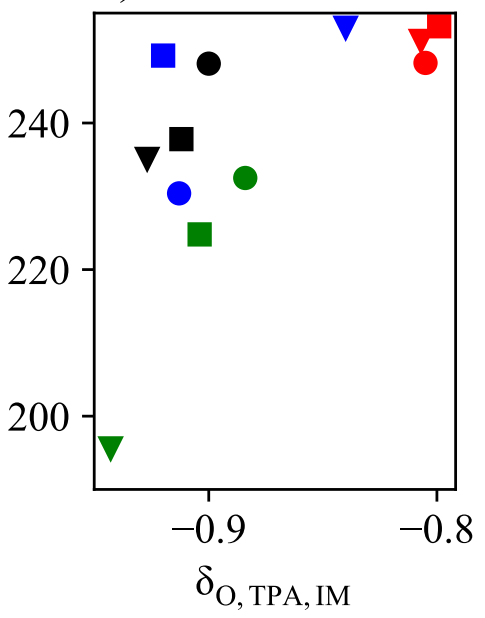

c)

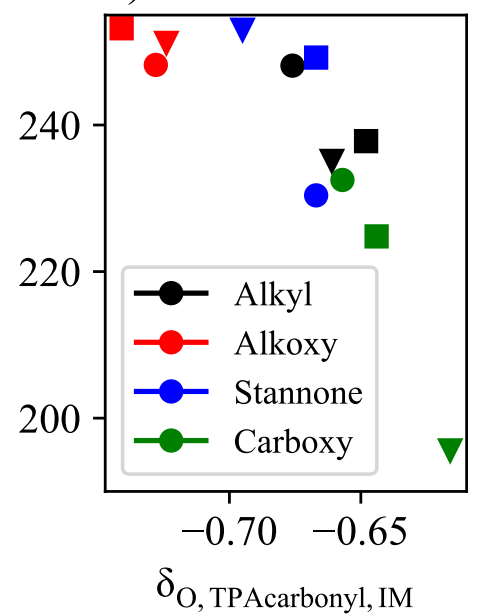

Figure S7: The influence of box transfer activation energy of different ligands depending on (a) the partial charge of tin during the transition state. (b) The partial charge of the TPA oxygen bonded to tin in the stable precursor. (c) The partial charge of the TPA carbonyl oxygen in the stable precursor. The shape indicates the number of carbon atoms, where a circle is 1 , square is 2 , and triangle is 4.

For the coordinated acyl transfer reaction in Figure S8b, we see that alkyl ligands are involved in reactions that are among the highest in activation energy. We will first note that the stable precursors in these reactions differ in that the alkoxy and carboxy series have both TPA oxygen atoms coordinated to the tin center, while for the alkyl and stannone series, the carbonyl oxygen is free. This difference in coordination occurs as the electron-withdrawing ligands of the alkoxy and carboxy series satisfy the stabilization needs of the tin center. However, now we cannot compare the partial charges of TPA's oxygen atoms to gain insight among the ligand series. We instead inspect the carbonyl carbon and find that some balance between the weak methyl ligand and the stronger electrophilic methoxy works together to lower the activation energy.

a)

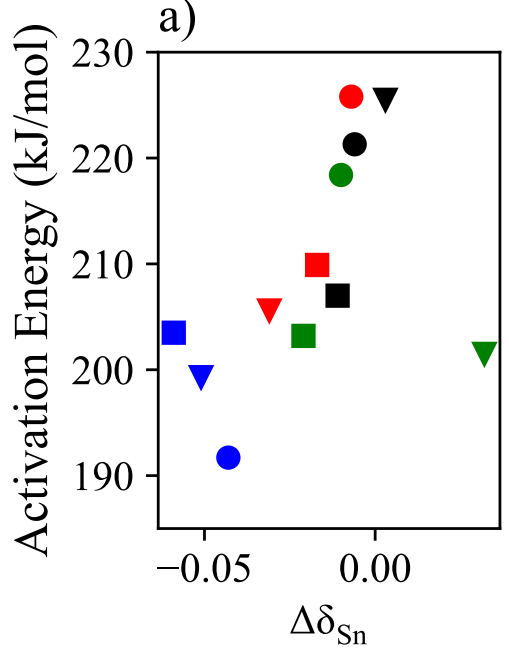

b)

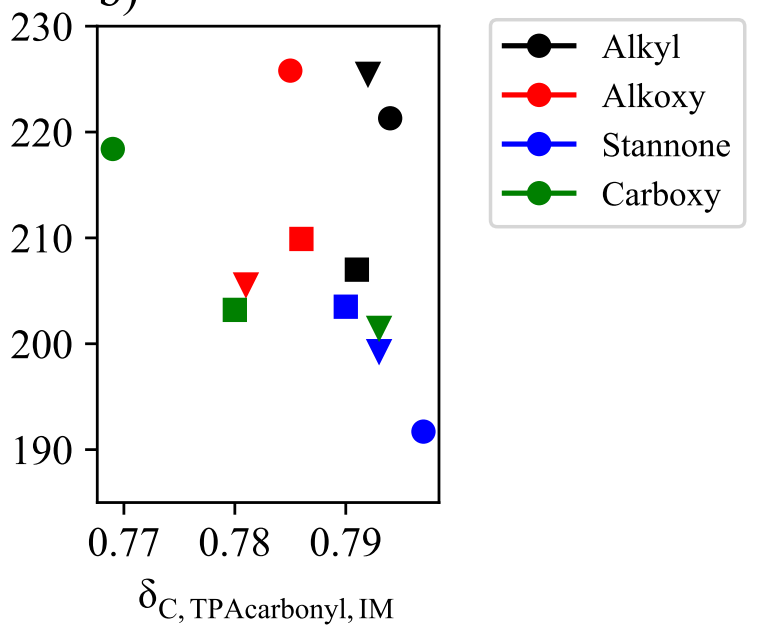

Figure S8: The influence of coordinated transfer activation energy of different ligands depending on (a) the change in partial charge of tin between the transition date and stable precursor. (b) The partial charge of the TPA carbonyl carbon in the stable precursor. The shape indicates the number of carbon atoms, where a circle is one, square is two, and triangle is four. 
S8: Box and Coordinated Transfer Mechanisms for Selected Metals with Methoxy Ligands To round out our discussion of the effect of different post-transition metal centers and the addition of electron-donating ligands, we tested transition metals with an oxidation state of three or four to support a methoxy ligand , and compared to the case where a methyl group is used. Figure S9 invariably shows that metals with an oxidation state of three favor coordinated transfer over box transfer. Methoxy ligands have an inhibiting effect in both box and coordinated transfer mechanisms. The electron withdrawal of the alkoxy ligands causes polarization in bonds around the metal center. Notice that the largest penalties occur for box transfer reactions, as the metal center must break a $\sigma$-bond to create a $\pi$ bond, while the coordinated transfer involves one $\sigma$-bond exchanged for another. In general, the activation energies decrease for group 13 metals as the period increases. As we shift our attention to metals with an oxidation state of four, we look at group 14

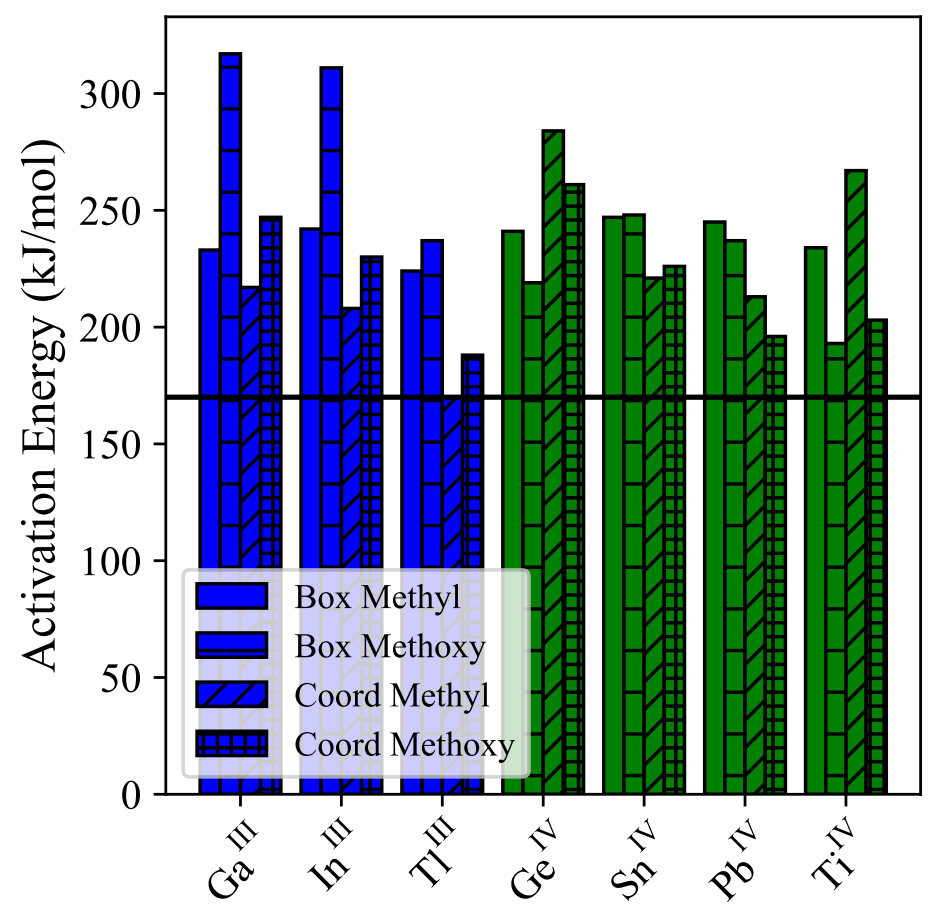

Figure S9: Comparison of intramolecular acyl box and coordinated transfer mechanisms for post-transition metals with oxidation state of three or four and either methyl or methoxy ligands. A black line marks the minimum activation energy as reference. atoms and titanium. In each case, methyl ligands are preferred, except in the case of Sn(IV). For all group 14 atoms, the coordinated transfer is preferred over box transfer, but the transition metal titanium has the reverse preference.

\section{S9: Optimized Structures of Reactions Involving Dimethyltin Oxide}

Here we present the minimized reaction structure coordinates used in the free energy calculations and partial charge comparisons. The stable state structures were produced in Gaussian 09 with the following route card or calculation instructions.

$$
\begin{aligned}
& \text { \# opt=(calcall, tight) wb97xd/gen pop }=(\text { nbo, savenbos }) \text { density } \\
& \text { scrf=(solvent=benzaldehyde, } p c m) \text { pseudo=read scf }=(\text { fermi, } x q c, \text { maxcyc }=500)
\end{aligned}
$$

For transition states, the following route card was used:

$$
\begin{aligned}
& \text { \# opt=(calcall, ts, noeigentest) wb97xd/gen pop=(nbo, savenbos }) \text { density } \\
& \text { scrf=(solvent=benzaldehyde, } p c m) \text { pseudo=read } s c f=(\text { fermi, } x q c, \text { maxcyc }=500)
\end{aligned}
$$

Where the basis sets from LANL2DZdp were read in as taken from Basis Set Exchange., ${ }^{2,3}$

Below the minimized coordinates and free energies of all reactions discussed in this work are provided with their free energy values at $500 \mathrm{~K}$ (computed with Arkane ${ }^{4}$ ). The negative vibrational frequencies of each transition state is also included. Two transition states, TS2b (i.e. structure 22) and TS4 (i.e. structure 27) have interestingly low frequencies, and so movies of their respective IRC curves have been provided as supplemental material. 
Table S5: Minimized structures used in this work

\begin{tabular}{|c|c|c|c|}
\hline \multicolumn{4}{|c|}{ 1) CBDO: Cyclobutanediol, $\mathrm{G}_{500 \mathrm{~K}}=-412.85202 \mathrm{~kJ} / \mathrm{mol}$} \\
\hline $\mathrm{O}$ & 2.265515 & 0.002311 & -0.373548 \\
\hline $\mathrm{C}$ & 1.076427 & 0.010376 & 0.387257 \\
\hline $\mathrm{C}$ & 0.006743 & -1.080779 & 0.134811 \\
\hline $\mathrm{C}$ & 0.001102 & 1.08852 & 0.106697 \\
\hline $\mathrm{H}$ & 1.391648 & 0.024391 & 1.435561 \\
\hline $\mathrm{H}$ & 0.002635 & -1.373253 & -0.924069 \\
\hline $\mathrm{C}$ & -1.066629 & -0.003082 & 0.375671 \\
\hline $\mathrm{H}$ & 0.006369 & -1.973399 & 0.768094 \\
\hline $\mathrm{H}$ & -1.396243 & 0.009668 & 1.423539 \\
\hline $\mathrm{O}$ & -2.179249 & -0.091152 & -0.494222 \\
\hline $\mathrm{H}$ & -0.004574 & 1.356468 & -0.958511 \\
\hline $\mathrm{H}$ & -0.003731 & 1.99451 & 0.721362 \\
\hline $\mathrm{H}$ & -2.81515 & 0.588968 & -0.24248 \\
\hline $\mathrm{H}$ & 2.02307 & -0.006836 & -1.307942 \\
\hline \multicolumn{4}{|c|}{ 2) $\mathrm{H}_{2} \mathrm{O}, \mathrm{G}_{500 \mathrm{~K}}=-326.008912188 \mathrm{~kJ} / \mathrm{mol}$} \\
\hline $\mathrm{O}$ & 0 & 0 & 0.118665 \\
\hline $\mathrm{H}$ & 0 & 0.76022 & -0.474661 \\
\hline $\mathrm{H}$ & 0 & -0.76022 & -0.474661 \\
\hline \multicolumn{4}{|c|}{ 3) $\mathrm{H}_{3} \mathrm{O}^{+}, \mathrm{G}_{500 \mathrm{~K}}=221.864968128 \mathrm{~kJ} / \mathrm{mol}$} \\
\hline $\mathrm{O}$ & 0.000148 & 0.000108 & -0.090339 \\
\hline $\mathrm{H}$ & 0.685923 & 0.615169 & 0.239661 \\
\hline $\mathrm{H}$ & -0.875272 & 0.286313 & 0.239734 \\
\hline $\mathrm{H}$ & 0.189217 & -0.901638 & 0.239701 \\
\hline \multicolumn{4}{|c|}{ 4) TPA: Terphthalic Acid, $\mathrm{G}_{500 \mathrm{~K}}=-722.38015 \mathrm{~kJ} / \mathrm{mol}$} \\
\hline $\mathrm{O}$ & 3.541883 & 1.11018 & 0.000002 \\
\hline $\mathrm{C}$ & 2.889933 & 0.082576 & 0.000001 \\
\hline $\mathrm{C}$ & 1.394372 & 0.035225 & 0.000001 \\
\hline $\mathrm{C}$ & 0.696891 & -1.180488 & -0.000001 \\
\hline $\mathrm{C}$ & 0.695858 & 1.250289 & 0.000001 \\
\hline $\mathrm{C}$ & -0.695858 & 1.250289 & 0.000001 \\
\hline $\mathrm{C}$ & -0.696891 & -1.180488 & -0.000001 \\
\hline $\mathrm{C}$ & -1.394372 & 0.035225 & -0.000001 \\
\hline $\mathrm{H}$ & 1.238234 & -2.122565 & -0.000001 \\
\hline $\mathrm{H}$ & -1.238234 & -2.122565 & -0.000002 \\
\hline $\mathrm{H}$ & 1.250159 & 2.185841 & 0.000002 \\
\hline $\mathrm{H}$ & -1.250159 & 2.185841 & 0.000001 \\
\hline $\mathrm{C}$ & -2.889933 & 0.082576 & -0.000001 \\
\hline
\end{tabular}




\begin{tabular}{|c|c|c|c|}
\hline $\mathrm{O}$ & -3.541883 & 1.11018 & 0 \\
\hline $\mathrm{O}$ & -3.456946 & -1.13288 & -0.000002 \\
\hline $\mathrm{H}$ & -4.419146 & -1.007288 & -0.000003 \\
\hline $\mathrm{O}$ & 3.456946 & -1.13288 & 0 \\
\hline $\mathrm{H}$ & 4.419146 & -1.007288 & 0.000001 \\
\hline \multicolumn{4}{|c|}{ 5) TPA- $\mathrm{H}_{3} \mathrm{O}^{+}$: Terphthalic Acid with $\mathrm{H}_{3} \mathrm{O}, \mathrm{G}_{500 \mathrm{~K}}=-461.01404 \mathrm{~kJ} / \mathrm{mol}$} \\
\hline $\mathrm{O}$ & -2.909749 & 1.989778 & -0.221924 \\
\hline $\mathrm{C}$ & -2.41257 & 0.894349 & -0.283774 \\
\hline $\mathrm{C}$ & -0.963478 & 0.576205 & -0.202604 \\
\hline $\mathrm{C}$ & -0.433773 & -0.593227 & -0.766589 \\
\hline $\mathrm{C}$ & -0.127339 & 1.503457 & 0.435643 \\
\hline $\mathrm{C}$ & 1.236859 & 1.248521 & 0.528931 \\
\hline $\mathrm{C}$ & 0.935564 & -0.838472 & -0.683564 \\
\hline $\mathrm{C}$ & 1.768932 & 0.07853 & -0.029768 \\
\hline $\mathrm{H}$ & -1.075257 & -1.296369 & -1.292408 \\
\hline $\mathrm{H}$ & 1.352644 & -1.737442 & -1.128488 \\
\hline $\mathrm{H}$ & -0.552233 & 2.409813 & 0.860342 \\
\hline $\mathrm{H}$ & 1.898591 & 1.950444 & 1.030383 \\
\hline $\mathrm{C}$ & 3.245002 & -0.150092 & 0.091828 \\
\hline $\mathrm{O}$ & 4.004072 & 0.604053 & 0.669088 \\
\hline $\mathrm{O}$ & 3.652209 & -1.278947 & -0.503699 \\
\hline $\mathrm{H}$ & 4.612612 & -1.34734 & -0.381501 \\
\hline $\mathrm{H}$ & -4.122992 & 0.082085 & -0.569751 \\
\hline $\mathrm{O}$ & -3.206503 & -0.223373 & -0.446319 \\
\hline $\mathrm{O}$ & -3.160152 & -2.287548 & 1.053658 \\
\hline $\mathrm{H}$ & -2.427382 & -2.263229 & 1.696055 \\
\hline $\mathrm{H}$ & -3.082624 & -3.101103 & 0.522329 \\
\hline $\mathrm{H}$ & -3.129528 & -1.457633 & 0.441548 \\
\hline \multicolumn{4}{|c|}{ 6) IM1: $\mathrm{Sn}\left(\mathrm{CH}_{3}\right)_{2}(=\mathrm{O}), \mathrm{G}_{500 \mathrm{~K}}=-203.39679 \mathrm{~kJ} / \mathrm{mol}$} \\
\hline $\mathrm{Sn}$ & -0.000455 & 0.089441 & -0.00067 \\
\hline $\mathrm{O}$ & 0.013111 & 1.906259 & 0.001575 \\
\hline $\mathrm{C}$ & -1.813925 & -1.021779 & 0.002353 \\
\hline $\mathrm{H}$ & -2.661897 & -0.350506 & 0.167079 \\
\hline $\mathrm{H}$ & -1.773879 & -1.780721 & 0.791689 \\
\hline $\mathrm{H}$ & -1.922948 & -1.524788 & -0.965718 \\
\hline $\mathrm{C}$ & 1.802393 & -1.041467 & -0.000972 \\
\hline $\mathrm{H}$ & 2.255264 & -0.982302 & 0.995722 \\
\hline $\mathrm{H}$ & 1.591928 & -2.087671 & -0.244233 \\
\hline $\mathrm{H}$ & 2.498559 & -0.61668 & -0.731938 \\
\hline
\end{tabular}




\begin{tabular}{|c|c|c|c|}
\hline \multicolumn{4}{|c|}{ 7) $\mathrm{IM} 1+: \mathrm{Sn}\left(\mathrm{CH}_{3}\right)_{2}\left(=\mathrm{O}\left(\mathrm{H}_{3} \mathrm{O}^{+}\right)\right), \mathrm{G}_{500 \mathrm{~K}}=-166.694744096 \mathrm{~kJ} / \mathrm{mol}$} \\
\hline $\mathrm{Sn}$ & 0.02389 & -0.001089 & 0.046256 \\
\hline $\mathrm{C}$ & -1.950632 & -0.70764 & -0.075931 \\
\hline $\mathrm{H}$ & -2.629524 & 0.143855 & 0.025124 \\
\hline $\mathrm{H}$ & -2.112631 & -1.433348 & 0.72787 \\
\hline $\mathrm{H}$ & -2.085926 & -1.199971 & -1.044544 \\
\hline $\mathrm{C}$ & 1.884751 & -0.963704 & -0.016385 \\
\hline $\mathrm{H}$ & 2.541541 & -0.49442 & 0.722371 \\
\hline $\mathrm{H}$ & 1.753491 & -2.027884 & 0.195589 \\
\hline $\mathrm{H}$ & 2.302567 & -0.829151 & -1.020248 \\
\hline $\mathrm{O}$ & 0.228965 & 1.857597 & 0.255326 \\
\hline $\mathrm{H}$ & -0.621095 & 2.356835 & 0.289032 \\
\hline $\mathrm{O}$ & -2.215672 & 3.053212 & 0.337619 \\
\hline $\mathrm{H}$ & -2.396106 & 3.5334 & 1.155472 \\
\hline $\mathrm{H}$ & -2.372928 & 3.697049 & -0.364354 \\
\hline \multicolumn{4}{|c|}{ 8) IM2: $\mathrm{Sn}\left(\mathrm{CH}_{3}\right)_{2}(\mathrm{OH})(\mathrm{CBDO}), \mathrm{G}_{500 \mathrm{~K}}=-756.559248436 \mathrm{~kJ} / \mathrm{mol}$} \\
\hline $\mathrm{Sn}$ & -1.361278 & -0.014497 & 0.019947 \\
\hline $\mathrm{O}$ & 0.323395 & 0.940089 & -0.246352 \\
\hline $\mathrm{C}$ & 1.473966 & 0.267616 & -0.685305 \\
\hline $\mathrm{C}$ & 2.149628 & -0.765817 & 0.258931 \\
\hline $\mathrm{C}$ & 2.78576 & 1.084395 & -0.665526 \\
\hline $\mathrm{H}$ & 1.315148 & -0.17686 & -1.681497 \\
\hline $\mathrm{H}$ & 1.962264 & -0.493137 & 1.305268 \\
\hline $\mathrm{C}$ & 3.511953 & -0.190471 & -0.201064 \\
\hline $\mathrm{H}$ & 1.950078 & -1.832085 & 0.100417 \\
\hline $\mathrm{H}$ & 3.916697 & -0.755088 & -1.052276 \\
\hline $\mathrm{O}$ & 4.519566 & 0.031991 & 0.768321 \\
\hline $\mathrm{H}$ & 2.750919 & 1.811285 & 0.155541 \\
\hline $\mathrm{H}$ & 3.118541 & 1.570362 & -1.588305 \\
\hline $\mathrm{H}$ & 4.942281 & -0.812518 & 0.964249 \\
\hline $\mathrm{C}$ & -1.524299 & -1.481318 & -1.507206 \\
\hline $\mathrm{H}$ & -0.737463 & -2.233942 & -1.379348 \\
\hline $\mathrm{H}$ & -1.410559 & -1.01026 & -2.490257 \\
\hline $\mathrm{H}$ & -2.501003 & -1.974828 & -1.456998 \\
\hline $\mathrm{O}$ & -1.200532 & -0.891332 & 1.759231 \\
\hline $\mathrm{H}$ & -0.640221 & -1.675988 & 1.773416 \\
\hline $\mathrm{C}$ & -2.817842 & 1.486524 & 0.304688 \\
\hline $\mathrm{H}$ & -3.749521 & 1.04042 & 0.6695 \\
\hline $\mathrm{H}$ & -2.45245 & 2.207799 & 1.044268 \\
\hline
\end{tabular}




\begin{tabular}{|c|c|c|c|}
\hline $\mathrm{H}$ & -3.015237 & 2.008147 & -0.638038 \\
\hline \multicolumn{4}{|c|}{ 9) IM3: $\mathrm{Sn}\left(\mathrm{CH}_{3}\right)_{2}(\mathrm{OH})(\mathrm{TPA}), \mathrm{G}_{500 \mathrm{~K}}=-1080.87782462 \mathrm{~kJ} / \mathrm{mol}$} \\
\hline Sn & -2.87 & 0.130549 & 0.003874 \\
\hline $\mathrm{O}$ & -3.164803 & -0.890476 & -1.61692 \\
\hline $\mathrm{C}$ & -3.302647 & -0.901523 & 1.798913 \\
\hline $\mathrm{H}$ & -3.155103 & -1.974859 & 1.644609 \\
\hline $\mathrm{H}$ & -4.336935 & -0.709467 & 2.103802 \\
\hline $\mathrm{H}$ & -2.624284 & -0.553833 & 2.586302 \\
\hline $\mathrm{C}$ & -3.700372 & 2.032792 & -0.378713 \\
\hline $\mathrm{H}$ & -4.788852 & 1.952044 & -0.473802 \\
\hline $\mathrm{H}$ & -3.463523 & 2.719368 & 0.44149 \\
\hline $\mathrm{H}$ & -3.287071 & 2.430654 & -1.311997 \\
\hline $\mathrm{O}$ & -0.88169 & 0.562887 & -0.005818 \\
\hline $\mathrm{C}$ & -0.138299 & -0.516707 & 0.011966 \\
\hline $\mathrm{O}$ & -0.627076 & -1.648907 & 0.016205 \\
\hline $\mathrm{C}$ & 1.343347 & -0.284247 & 0.024199 \\
\hline $\mathrm{C}$ & 2.201666 & -1.389737 & 0.028574 \\
\hline $\mathrm{C}$ & 1.869434 & 1.01481 & 0.034208 \\
\hline $\mathrm{C}$ & 3.248848 & 1.204953 & 0.046927 \\
\hline $\mathrm{C}$ & 3.582273 & -1.200745 & 0.028012 \\
\hline $\mathrm{C}$ & 4.109387 & 0.098829 & 0.031853 \\
\hline $\mathrm{H}$ & 1.781143 & -2.392375 & 0.035151 \\
\hline $\mathrm{H}$ & 4.232217 & -2.074425 & 0.0575 \\
\hline $\mathrm{H}$ & 1.195644 & 1.86756 & 0.032774 \\
\hline $\mathrm{H}$ & 3.669708 & 2.207629 & 0.060605 \\
\hline $\mathrm{C}$ & 5.588329 & 0.360344 & 0.041425 \\
\hline $\mathrm{O}$ & 6.083635 & 1.36027 & 0.518614 \\
\hline $\mathrm{O}$ & 6.383298 & -0.571127 & -0.514929 \\
\hline $\mathrm{H}$ & 5.859061 & -1.256033 & -0.953298 \\
\hline $\mathrm{H}$ & -2.740693 & -1.757512 & -1.598231 \\
\hline \multicolumn{4}{|c|}{ 10) $\mathrm{IM} 3+: \mathrm{Sn}\left(\mathrm{CH}_{3}\right)_{2}(\mathrm{OH})\left(\mathrm{TPA}\left(\mathrm{H}_{3} \mathrm{O}^{+}\right)\right), \mathrm{G}_{500 \mathrm{~K}}=-943.567312543 \mathrm{~kJ} / \mathrm{mol}$} \\
\hline $\mathrm{Sn}$ & 2.346819 & -0.425837 & -0.073533 \\
\hline $\mathrm{O}$ & 3.19486 & 1.565728 & -0.206821 \\
\hline $\mathrm{C}$ & 3.000848 & -0.913911 & 1.86095 \\
\hline $\mathrm{H}$ & 4.07634 & -0.730564 & 1.947273 \\
\hline $\mathrm{H}$ & 2.461104 & -0.28698 & 2.578556 \\
\hline $\mathrm{H}$ & 2.787927 & -1.968699 & 2.059881 \\
\hline $\mathrm{C}$ & 2.937735 & -1.044679 & -1.988447 \\
\hline $\mathrm{H}$ & 2.347979 & -0.492794 & -2.727912 \\
\hline
\end{tabular}




\begin{tabular}{|c|c|c|c|}
\hline $\mathrm{H}$ & 4.001282 & -0.831259 & -2.133448 \\
\hline $\mathrm{H}$ & 2.755352 & -2.118017 & -2.097249 \\
\hline $\mathrm{O}$ & 0.595895 & 0.69868 & -0.072941 \\
\hline C & -0.192302 & -0.325023 & -0.02074 \\
\hline C & -1.658727 & -0.145342 & 0.00769 \\
\hline $\mathrm{C}$ & -2.206472 & 1.143035 & -0.021861 \\
\hline $\mathrm{C}$ & -2.486516 & -1.2753 & 0.068911 \\
\hline $\mathrm{C}$ & -3.867593 & -1.113315 & 0.101397 \\
\hline $\mathrm{C}$ & -3.590561 & 1.301562 & -0.002169 \\
\hline $\mathrm{C}$ & -4.421456 & 0.173671 & 0.05471 \\
\hline $\mathrm{H}$ & -1.553261 & 2.011118 & -0.055451 \\
\hline $\mathrm{H}$ & -4.004555 & 2.308531 & 0.000761 \\
\hline $\mathrm{H}$ & -2.043889 & -2.267951 & 0.093888 \\
\hline $\mathrm{H}$ & -4.525202 & -1.977371 & 0.156605 \\
\hline $\mathrm{C}$ & -5.921195 & 0.291198 & 0.097992 \\
\hline $\mathrm{O}$ & -6.630575 & -0.530409 & 0.638256 \\
\hline $\mathrm{O}$ & -6.473992 & 1.361783 & -0.495706 \\
\hline $\mathrm{H}$ & -5.811181 & 1.873863 & -0.979805 \\
\hline $\mathrm{O}$ & 0.357638 & -1.462028 & 0.003582 \\
\hline $\mathrm{H}$ & 2.653564 & 2.305181 & 0.105208 \\
\hline $\mathrm{H}$ & 4.149447 & 1.748508 & 0.064071 \\
\hline $\mathrm{O}$ & 5.642621 & 2.066217 & 0.484831 \\
\hline $\mathrm{H}$ & 6.277956 & 2.086835 & -0.242478 \\
\hline $\mathrm{H}$ & 6.032071 & 1.480317 & 1.14656 \\
\hline \multicolumn{4}{|c|}{ 11) IM4: $\mathrm{Sn}\left(\mathrm{CH}_{3}\right)_{2}(\mathrm{CBDO})_{2}, \mathrm{G}_{500 \mathrm{~K}}=-842.929560486 \mathrm{~kJ} / \mathrm{mol}$} \\
\hline $\mathrm{Sn}$ & -2.278958 & -0.341564 & 1.951323 \\
\hline $\mathrm{C}$ & -1.765699 & -1.931676 & 3.24669 \\
\hline $\mathrm{H}$ & -1.070356 & -1.578688 & 4.016638 \\
\hline $\mathrm{H}$ & -1.283448 & -2.726274 & 2.666243 \\
\hline $\mathrm{H}$ & -2.66233 & -2.334443 & 3.730647 \\
\hline $\mathrm{C}$ & -3.277221 & 1.376294 & 2.707468 \\
\hline $\mathrm{H}$ & -3.520103 & 2.060938 & 1.886878 \\
\hline $\mathrm{H}$ & -4.20247 & 1.085749 & 3.217411 \\
\hline $\mathrm{H}$ & -2.626212 & 1.895143 & 3.420986 \\
\hline $\mathrm{O}$ & -3.243741 & -1.229615 & 0.516554 \\
\hline $\mathrm{C}$ & -4.004585 & -0.629255 & -0.495708 \\
\hline $\mathrm{C}$ & -3.53001 & 0.687224 & -1.166668 \\
\hline $\mathrm{C}$ & -5.336129 & 0.078765 & -0.131612 \\
\hline $\mathrm{H}$ & -4.183779 & -1.393999 & -1.263466 \\
\hline
\end{tabular}




\begin{tabular}{|c|c|c|c|}
\hline $\mathrm{H}$ & -2.99026 & 1.323585 & -0.452904 \\
\hline $\mathrm{C}$ & -5.003026 & 1.128511 & -1.221994 \\
\hline $\mathrm{H}$ & -2.961338 & 0.625494 & -2.099572 \\
\hline $\mathrm{H}$ & -5.468365 & 0.874743 & -2.184094 \\
\hline $\mathrm{O}$ & -5.198695 & 2.498735 & -0.921806 \\
\hline $\mathrm{H}$ & -5.269657 & 0.542334 & 0.861034 \\
\hline $\mathrm{H}$ & -6.268065 & -0.489717 & -0.216698 \\
\hline $\mathrm{H}$ & -6.140314 & 2.693369 & -0.997447 \\
\hline $\mathrm{O}$ & -0.65511 & 0.263202 & 1.045494 \\
\hline $\mathrm{C}$ & -0.023712 & 1.475479 & 1.361464 \\
\hline $\mathrm{C}$ & -0.592507 & 2.79777 & 0.776029 \\
\hline $\mathrm{C}$ & 1.299377 & 1.762438 & 0.617237 \\
\hline $\mathrm{H}$ & 0.126063 & 1.581716 & 2.448581 \\
\hline $\mathrm{H}$ & -1.076293 & 2.598854 & -0.188675 \\
\hline $\mathrm{C}$ & 0.85921 & 3.245329 & 0.535149 \\
\hline $\mathrm{H}$ & -1.237814 & 3.423024 & 1.402264 \\
\hline $\mathrm{H}$ & 1.26343 & 1.30718 & -0.380285 \\
\hline $\mathrm{H}$ & 2.244348 & 1.519186 & 1.114422 \\
\hline $\mathrm{H}$ & 1.247421 & 3.824841 & 1.384157 \\
\hline $\mathrm{O}$ & 1.0571 & 3.950578 & -0.676666 \\
\hline $\mathrm{H}$ & 1.980352 & 4.226213 & -0.718386 \\
\hline \multicolumn{4}{|c|}{ 12) IM5: $\mathrm{Sn}\left(\mathrm{CH}_{3}\right)_{2}(\mathrm{CBDO})(\mathrm{TPA}), \mathrm{G}_{500 \mathrm{~K}}=-1163.70010467 \mathrm{~kJ} / \mathrm{mol}$} \\
\hline $\mathrm{Sn}$ & -2.186544 & -1.304165 & 0.122085 \\
\hline $\mathrm{O}$ & -2.844756 & -0.05213 & -1.185867 \\
\hline $\mathrm{C}$ & -3.181487 & 1.308077 & -1.115143 \\
\hline $\mathrm{C}$ & -2.112512 & 2.363525 & -1.4856 \\
\hline $\mathrm{C}$ & -3.369277 & 2.035381 & 0.241632 \\
\hline $\mathrm{H}$ & -4.07836 & 1.451349 & -1.73379 \\
\hline $\mathrm{O}$ & -0.17082 & -1.190765 & -0.036941 \\
\hline $\mathrm{C}$ & 0.417935 & -0.113887 & 0.428183 \\
\hline $\mathrm{C}$ & 1.909479 & -0.080935 & 0.253183 \\
\hline $\mathrm{C}$ & 2.611821 & 1.060262 & 0.656835 \\
\hline $\mathrm{C}$ & 2.596081 & -1.163364 & -0.312867 \\
\hline $\mathrm{C}$ & 3.978317 & -1.103624 & -0.471492 \\
\hline $\mathrm{C}$ & 3.995847 & 1.118153 & 0.505641 \\
\hline $\mathrm{C}$ & 4.684861 & 0.033186 & -0.055765 \\
\hline $\mathrm{H}$ & 2.067999 & 1.900503 & 1.081544 \\
\hline $\mathrm{H}$ & 4.514312 & 2.029797 & 0.799836 \\
\hline $\mathrm{H}$ & 2.044737 & -2.045387 & -0.627511 \\
\hline
\end{tabular}




\begin{tabular}{|c|c|c|c|}
\hline $\mathrm{H}$ & 4.520067 & -1.936407 & -0.913679 \\
\hline $\mathrm{C}$ & 6.173666 & 0.042836 & -0.255264 \\
\hline $\mathrm{O}$ & 6.737349 & -0.645117 & -1.0814 \\
\hline $\mathrm{O}$ & 6.900214 & 0.856914 & 0.530966 \\
\hline $\mathrm{H}$ & 6.345755 & 1.272635 & 1.205802 \\
\hline $\mathrm{H}$ & -1.129605 & 2.038999 & -1.122944 \\
\hline $\mathrm{C}$ & -2.772964 & 3.285206 & -0.43035 \\
\hline $\mathrm{H}$ & -2.051099 & 2.706249 & -2.523922 \\
\hline $\mathrm{H}$ & -3.557808 & 3.907559 & -0.8828 \\
\hline $\mathrm{O}$ & -1.950251 & 4.068106 & 0.414724 \\
\hline $\mathrm{H}$ & -2.649313 & 1.663517 & 0.981416 \\
\hline $\mathrm{H}$ & -4.372322 & 2.093165 & 0.676516 \\
\hline $\mathrm{H}$ & -1.647804 & 4.834472 & -0.086634 \\
\hline $\mathrm{C}$ & -2.483568 & -3.181475 & -0.793511 \\
\hline $\mathrm{H}$ & -2.012834 & -3.178594 & -1.782873 \\
\hline $\mathrm{H}$ & -2.03556 & -3.974372 & -0.184411 \\
\hline $\mathrm{H}$ & -3.555001 & -3.379401 & -0.906966 \\
\hline $\mathrm{C}$ & -2.803746 & -0.967664 & 2.118256 \\
\hline $\mathrm{H}$ & -2.69108 & -1.893431 & 2.692873 \\
\hline $\mathrm{H}$ & -2.194391 & -0.176882 & 2.56513 \\
\hline $\mathrm{H}$ & -3.857523 & -0.667582 & 2.123856 \\
\hline $\mathrm{O}$ & -0.196697 & 0.80649 & 0.962502 \\
\hline \multicolumn{4}{|c|}{ 13) IM5+: $\mathrm{Sn}\left(\mathrm{CH}_{3}\right)_{2}(\mathrm{CBDO})\left(\mathrm{TPA}\left(\mathrm{H}_{3} \mathrm{O}^{+}\right)\right), \mathrm{G}_{500 \mathrm{~K}}=-944.717912544 \mathrm{~kJ} / \mathrm{mol}$} \\
\hline $\mathrm{Sn}$ & 2.183585 & -0.691395 & -1.050065 \\
\hline $\mathrm{C}$ & 0.660014 & 1.445982 & 0.304147 \\
\hline $\mathrm{C}$ & -0.494993 & 2.460573 & 0.213067 \\
\hline $\mathrm{C}$ & 1.625544 & 2.609659 & 0.632547 \\
\hline $\mathrm{H}$ & 0.530099 & 0.783758 & 1.172725 \\
\hline $\mathrm{O}$ & 0.962839 & -2.312503 & -0.101458 \\
\hline $\mathrm{C}$ & -0.03672 & -2.239513 & 0.858014 \\
\hline $\mathrm{C}$ & -1.221971 & -1.439987 & 0.456613 \\
\hline $\mathrm{C}$ & -2.041269 & -0.93372 & 1.474083 \\
\hline $\mathrm{C}$ & -1.547954 & -1.229078 & -0.889179 \\
\hline $\mathrm{C}$ & -2.688882 & -0.50335 & -1.215961 \\
\hline $\mathrm{C}$ & -3.169851 & -0.188091 & 1.144592 \\
\hline $\mathrm{C}$ & -3.492483 & 0.031874 & -0.2021 \\
\hline $\mathrm{H}$ & -1.790163 & -1.12134 & 2.514914 \\
\hline $\mathrm{H}$ & -3.8035 & 0.184905 & 1.947411 \\
\hline $\mathrm{H}$ & -0.933173 & -1.647354 & -1.680824 \\
\hline
\end{tabular}




\begin{tabular}{|c|c|c|c|}
\hline $\mathrm{H}$ & -2.959152 & -0.341391 & -2.25612 \\
\hline $\mathrm{C}$ & -4.708659 & 0.815518 & -0.615864 \\
\hline $\mathrm{O}$ & -5.281906 & 0.631495 & -1.668253 \\
\hline $\mathrm{O}$ & -5.157884 & 1.755165 & 0.23196 \\
\hline $\mathrm{H}$ & -4.556168 & 1.869586 & 0.980634 \\
\hline $\mathrm{H}$ & -0.603167 & 2.809034 & -0.820858 \\
\hline $\mathrm{C}$ & 0.390779 & 3.445506 & 1.019449 \\
\hline $\mathrm{H}$ & -1.467639 & 2.171727 & 0.623061 \\
\hline $\mathrm{H}$ & 0.183196 & 3.38139 & 2.096104 \\
\hline $\mathrm{O}$ & 0.456283 & 4.794348 & 0.602738 \\
\hline $\mathrm{H}$ & 2.063607 & 3.001777 & -0.293737 \\
\hline $\mathrm{H}$ & 2.404815 & 2.448964 & 1.384252 \\
\hline $\mathrm{H}$ & -0.323833 & 5.253934 & 0.935261 \\
\hline $\mathrm{C}$ & 2.184749 & -1.404903 & -3.020621 \\
\hline $\mathrm{H}$ & 1.218985 & -1.181747 & -3.484184 \\
\hline $\mathrm{H}$ & 2.98571 & -0.909206 & -3.578511 \\
\hline $\mathrm{H}$ & 2.359918 & -2.485501 & -3.014543 \\
\hline $\mathrm{C}$ & 3.714812 & -0.670564 & 0.392166 \\
\hline $\mathrm{H}$ & 4.397221 & -1.508097 & 0.219613 \\
\hline $\mathrm{H}$ & 4.261593 & 0.275208 & 0.318077 \\
\hline $\mathrm{H}$ & 3.262607 & -0.757345 & 1.385791 \\
\hline $\mathrm{O}$ & 0.892021 & 0.695368 & -0.87231 \\
\hline $\mathrm{O}$ & 0.101781 & -2.818061 & 1.905659 \\
\hline $\mathrm{H}$ & 2.696476 & -4.320743 & 1.373922 \\
\hline $\mathrm{O}$ & 2.645753 & -4.112395 & 0.4307 \\
\hline $\mathrm{H}$ & 1.601613 & -3.091208 & 0.160051 \\
\hline $\mathrm{H}$ & 2.495364 & -4.95828 & -0.012965 \\
\hline \multicolumn{4}{|c|}{ 14) $\mathrm{IM} 5 \mathrm{~d}+: \mathrm{Sn}\left(\mathrm{CH}_{3}\right)_{2}(\mathrm{CBDO})\left(\mathrm{TPA}\left(\mathrm{H}_{3} \mathrm{O}^{+}\right)\right), \mathrm{G}_{500 \mathrm{~K}}=-989.55784057 \mathrm{~kJ} / \mathrm{mol}$} \\
\hline $\mathrm{Sn}$ & 1.859496 & -1.526311 & 0.223293 \\
\hline $\mathrm{C}$ & 2.974988 & 1.113219 & -0.473181 \\
\hline $\mathrm{C}$ & 3.527481 & 2.050564 & -1.566944 \\
\hline $\mathrm{C}$ & 4.370609 & 1.127872 & 0.201715 \\
\hline $\mathrm{H}$ & 2.232195 & 1.631211 & 0.150323 \\
\hline $\mathrm{O}$ & -0.212176 & -1.154989 & 0.116244 \\
\hline $\mathrm{C}$ & -0.80116 & -0.096712 & 0.458352 \\
\hline $\mathrm{C}$ & -2.25636 & 0.025979 & 0.217758 \\
\hline $\mathrm{C}$ & -3.053868 & 0.850865 & 1.021497 \\
\hline $\mathrm{C}$ & -2.827189 & -0.733789 & -0.815449 \\
\hline $\mathrm{C}$ & -4.192994 & -0.645953 & -1.057489 \\
\hline
\end{tabular}




\begin{tabular}{|c|c|c|c|}
\hline $\mathrm{C}$ & -4.423028 & 0.932779 & 0.776169 \\
\hline $\mathrm{C}$ & -4.991622 & 0.193291 & -0.268512 \\
\hline $\mathrm{H}$ & -2.628407 & 1.405277 & 1.854016 \\
\hline $\mathrm{H}$ & -5.035178 & 1.549541 & 1.431673 \\
\hline $\mathrm{H}$ & -2.199199 & -1.377645 & -1.425599 \\
\hline $\mathrm{H}$ & -4.649807 & -1.224687 & -1.856328 \\
\hline $\mathrm{C}$ & -6.468463 & 0.231848 & -0.563671 \\
\hline $\mathrm{O}$ & -7.064092 & -0.709289 & -1.041282 \\
\hline $\mathrm{O}$ & -7.127924 & 1.365257 & -0.276917 \\
\hline $\mathrm{H}$ & -6.520396 & 2.069243 & -0.010006 \\
\hline $\mathrm{H}$ & 3.918932 & 1.455504 & -2.400834 \\
\hline $\mathrm{C}$ & 4.676557 & 2.411383 & -0.590789 \\
\hline $\mathrm{H}$ & 2.881786 & 2.85206 & -1.939786 \\
\hline $\mathrm{H}$ & 4.439174 & 3.311507 & -0.007532 \\
\hline $\mathrm{O}$ & 5.997626 & 2.478395 & -1.091234 \\
\hline $\mathrm{H}$ & 4.97984 & 0.301286 & -0.185946 \\
\hline $\mathrm{H}$ & 4.428869 & 1.162428 & 1.294592 \\
\hline $\mathrm{H}$ & 6.116978 & 3.334055 & -1.519933 \\
\hline $\mathrm{C}$ & 1.875688 & -3.34115 & -0.828592 \\
\hline $\mathrm{H}$ & 1.67048 & -3.140123 & -1.885031 \\
\hline $\mathrm{H}$ & 2.857656 & -3.814728 & -0.728985 \\
\hline $\mathrm{H}$ & 1.107957 & -4.007633 & -0.422202 \\
\hline $\mathrm{C}$ & 2.344593 & -1.189931 & 2.245955 \\
\hline $\mathrm{H}$ & 1.7152 & -1.814331 & 2.887906 \\
\hline $\mathrm{H}$ & 3.39743 & -1.439793 & 2.412648 \\
\hline $\mathrm{H}$ & 2.177415 & -0.133266 & 2.477962 \\
\hline $\mathrm{O}$ & 2.464562 & -0.11939 & -0.936841 \\
\hline $\mathrm{O}$ & -0.109326 & 0.84139 & 1.014731 \\
\hline $\mathrm{H}$ & -0.55295 & 1.753946 & 1.114715 \\
\hline $\mathrm{O}$ & -0.980975 & 3.224679 & 1.325359 \\
\hline $\mathrm{H}$ & -0.255373 & 3.784882 & 1.632304 \\
\hline $\mathrm{H}$ & -1.330361 & 3.666786 & 0.540004 \\
\hline \multicolumn{4}{|c|}{ 15) IM6: $\mathrm{Sn}\left(\mathrm{CH}_{3}\right)_{2}(\mathrm{TPA})_{2}, \mathrm{G}_{500 \mathrm{~K}}=-1494.84278486 \mathrm{~kJ} / \mathrm{mol}$} \\
\hline $\mathrm{Sn}$ & 0.080535 & -2.164104 & -0.044441 \\
\hline $\mathrm{O}$ & -1.46499 & -0.161475 & 1.65839 \\
\hline $\mathrm{O}$ & -1.66967 & -1.168569 & -0.328872 \\
\hline $\mathrm{C}$ & -2.09081 & -0.366988 & 0.620833 \\
\hline $\mathrm{C}$ & -3.414789 & 0.292999 & 0.347015 \\
\hline $\mathrm{C}$ & -3.955843 & 1.151196 & 1.310941 \\
\hline
\end{tabular}




\begin{tabular}{|c|c|c|c|}
\hline $\mathrm{C}$ & -4.10702 & 0.057577 & -0.848644 \\
\hline $\mathrm{C}$ & -5.331652 & 0.680085 & -1.078453 \\
\hline $\mathrm{C}$ & -5.176101 & 1.782856 & 1.078546 \\
\hline $\mathrm{C}$ & -5.865244 & 1.55386 & -0.121362 \\
\hline $\mathrm{H}$ & -3.418538 & 1.315203 & 2.241883 \\
\hline $\mathrm{H}$ & -5.59071 & 2.42335 & 1.855875 \\
\hline $\mathrm{H}$ & -3.683658 & -0.612864 & -1.591755 \\
\hline $\mathrm{H}$ & -5.87739 & 0.50139 & -2.002048 \\
\hline $\mathrm{C}$ & -7.186761 & 2.201879 & -0.418804 \\
\hline $\mathrm{O}$ & -8.010615 & 1.719713 & -1.16861 \\
\hline $\mathrm{O}$ & -7.44926 & 3.376165 & 0.182894 \\
\hline $\mathrm{H}$ & -6.67462 & 3.699171 & 0.664154 \\
\hline $\mathrm{C}$ & -0.034444 & -3.069091 & 1.853496 \\
\hline $\mathrm{H}$ & 0.381718 & -2.381853 & 2.596757 \\
\hline $\mathrm{H}$ & -1.082376 & -3.2724 & 2.097111 \\
\hline $\mathrm{H}$ & 0.536699 & -4.003045 & 1.85045 \\
\hline $\mathrm{C}$ & 0.164185 & -3.12493 & -1.917391 \\
\hline $\mathrm{H}$ & 0.417655 & -2.385941 & -2.68566 \\
\hline $\mathrm{H}$ & 0.926854 & -3.909508 & -1.904483 \\
\hline $\mathrm{H}$ & -0.811892 & -3.564089 & -2.149719 \\
\hline $\mathrm{O}$ & 1.144003 & -0.395653 & -0.126655 \\
\hline $\mathrm{C}$ & 2.348459 & -0.864527 & -0.014709 \\
\hline $\mathrm{O}$ & 2.497318 & -2.100038 & 0.105624 \\
\hline $\mathrm{C}$ & 3.491393 & 0.087215 & -0.036762 \\
\hline $\mathrm{C}$ & 4.792406 & -0.405275 & 0.117412 \\
\hline $\mathrm{C}$ & 3.265968 & 1.459952 & -0.206071 \\
\hline $\mathrm{C}$ & 5.872275 & 0.474689 & 0.09047 \\
\hline $\mathrm{H}$ & 4.952034 & -1.470727 & 0.262765 \\
\hline $\mathrm{C}$ & 4.345106 & 2.339038 & -0.22403 \\
\hline $\mathrm{H}$ & 2.250725 & 1.830679 & -0.321517 \\
\hline $\mathrm{C}$ & 5.650732 & 1.847827 & -0.086504 \\
\hline $\mathrm{H}$ & 6.874935 & 0.07729 & 0.240566 \\
\hline $\mathrm{H}$ & 4.186382 & 3.40714 & -0.350878 \\
\hline $\mathrm{C}$ & 6.778124 & 2.842285 & -0.112492 \\
\hline $\mathrm{O}$ & 6.641152 & 4.00543 & 0.203972 \\
\hline $\mathrm{O}$ & 7.984035 & 2.401442 & -0.51023 \\
\hline $\mathrm{H}$ & 7.933733 & 1.491429 & -0.8345 \\
\hline
\end{tabular}

16) IM6+: $\mathrm{Sn}\left(\mathrm{CH}_{3}\right)_{2}(\mathrm{TPA})\left(\mathrm{TPA}\left(\mathrm{H}_{3} \mathrm{O}^{+}\right)\right), \mathrm{G}_{500 \mathrm{~K}}=-1317.78845676 \mathrm{~kJ} / \mathrm{mol}$ $\mathrm{Sn}$ 0.291952 $-2.185401$ $-0.243947$ 


\begin{tabular}{|c|c|c|c|}
\hline $\mathrm{O}$ & -1.5108 & -1.041 & -0.389614 \\
\hline $\mathrm{C}$ & -2.030719 & -0.272007 & 0.454417 \\
\hline $\mathrm{C}$ & -3.357868 & 0.322036 & 0.161239 \\
\hline $\mathrm{C}$ & -3.737181 & 1.558685 & 0.698277 \\
\hline C & -4.222568 & -0.381911 & -0.691091 \\
\hline $\mathrm{C}$ & -5.478482 & 0.138955 & -0.979015 \\
\hline $\mathrm{C}$ & -4.985726 & 2.091457 & 0.381595 \\
\hline $\mathrm{C}$ & -5.857891 & 1.381416 & -0.452892 \\
\hline $\mathrm{H}$ & -3.065775 & 2.122342 & 1.341175 \\
\hline $\mathrm{H}$ & -5.272207 & 3.046375 & 0.818626 \\
\hline $\mathrm{H}$ & -3.911956 & -1.335199 & -1.110439 \\
\hline $\mathrm{H}$ & -6.167382 & -0.406164 & -1.619326 \\
\hline $\mathrm{C}$ & -7.229409 & 1.897916 & -0.797822 \\
\hline $\mathrm{O}$ & -8.167208 & 1.164415 & -1.025124 \\
\hline $\mathrm{O}$ & -7.39342 & 3.229316 & -0.848327 \\
\hline $\mathrm{H}$ & -6.547413 & 3.69065 & -0.761308 \\
\hline $\mathrm{C}$ & 0.037269 & -3.016395 & 1.668773 \\
\hline $\mathrm{H}$ & 0.375221 & -2.288061 & 2.412813 \\
\hline $\mathrm{H}$ & -1.019848 & -3.247517 & 1.834091 \\
\hline $\mathrm{H}$ & 0.633361 & -3.930759 & 1.748512 \\
\hline $\mathrm{C}$ & 0.106175 & -2.979418 & -2.176049 \\
\hline $\mathrm{H}$ & 0.254726 & -2.174011 & -2.903102 \\
\hline $\mathrm{H}$ & 0.863337 & -3.754638 & -2.327005 \\
\hline $\mathrm{H}$ & -0.893889 & -3.405925 & -2.302106 \\
\hline $\mathrm{O}$ & 1.253899 & -0.342237 & -0.213683 \\
\hline $\mathrm{C}$ & 2.450661 & -0.830196 & -0.186359 \\
\hline $\mathrm{O}$ & 2.555503 & -2.087438 & -0.192048 \\
\hline $\mathrm{C}$ & 3.622943 & 0.071898 & -0.147731 \\
\hline $\mathrm{C}$ & 4.910312 & -0.478185 & -0.158989 \\
\hline $\mathrm{C}$ & 3.437845 & 1.460194 & -0.08917 \\
\hline $\mathrm{C}$ & 6.018851 & 0.3649 & -0.123614 \\
\hline $\mathrm{H}$ & 5.038552 & -1.557145 & -0.192219 \\
\hline $\mathrm{C}$ & 4.546803 & 2.299176 & -0.038664 \\
\hline $\mathrm{H}$ & 2.432599 & 1.873488 & -0.079004 \\
\hline $\mathrm{C}$ & 5.837983 & 1.753971 & -0.066176 \\
\hline $\mathrm{H}$ & 7.013349 & -0.078028 & -0.107712 \\
\hline $\mathrm{H}$ & 4.422126 & 3.377685 & 0.017998 \\
\hline $\mathrm{C}$ & 7.000408 & 2.707799 & -0.003453 \\
\hline $\mathrm{O}$ & 6.92991 & 3.797271 & 0.524529 \\
\hline
\end{tabular}




\begin{tabular}{|c|c|c|c|}
\hline $\mathrm{O}$ & 8.155311 & 2.313242 & -0.56429 \\
\hline $\mathrm{H}$ & 8.045167 & 1.478169 & -1.040148 \\
\hline $\mathrm{O}$ & -1.393148 & -0.023672 & 1.551919 \\
\hline $\mathrm{H}$ & -1.916307 & 0.444791 & 2.284788 \\
\hline $\mathrm{O}$ & -2.526826 & 1.093645 & 3.574495 \\
\hline $\mathrm{H}$ & -1.949816 & 1.007341 & 4.345254 \\
\hline $\mathrm{H}$ & -3.376989 & 0.727274 & 3.852219 \\
\hline \multicolumn{4}{|c|}{ 17) IM7: $\mathrm{Sn}\left(\mathrm{CH}_{3}\right)_{2}(\mathrm{CBDO})(\mathrm{TPA})$ Orthoester, $\mathrm{G}_{500 \mathrm{~K}}=-1025.82893659 \mathrm{~kJ} / \mathrm{mol}$} \\
\hline $\mathrm{Sn}$ & 2.837236 & -0.778238 & -0.143548 \\
\hline $\mathrm{O}$ & 1.445855 & -0.623266 & 1.270324 \\
\hline $\mathrm{C}$ & 0.049493 & 2.659643 & 0.786791 \\
\hline $\mathrm{C}$ & -1.481715 & 2.864812 & 0.917475 \\
\hline $\mathrm{C}$ & -0.059611 & 3.026878 & -0.712156 \\
\hline $\mathrm{H}$ & 0.558852 & 3.470554 & 1.319097 \\
\hline $\mathrm{O}$ & 1.376235 & 0.445112 & -0.704527 \\
\hline $\mathrm{C}$ & 0.695474 & 0.292404 & 0.513065 \\
\hline $\mathrm{C}$ & -0.722494 & -0.251428 & 0.31741 \\
\hline $\mathrm{C}$ & -1.476839 & -0.58902 & 1.448575 \\
\hline $\mathrm{C}$ & -1.273216 & -0.422535 & -0.953635 \\
\hline $\mathrm{C}$ & -2.571923 & -0.910579 & -1.098196 \\
\hline $\mathrm{C}$ & -2.766492 & -1.091753 & 1.309823 \\
\hline $\mathrm{C}$ & -3.324018 & -1.256627 & 0.03049 \\
\hline $\mathrm{H}$ & -1.053283 & -0.443851 & 2.439647 \\
\hline $\mathrm{H}$ & -3.337572 & -1.31589 & 2.210586 \\
\hline $\mathrm{H}$ & -0.681193 & -0.168476 & -1.829061 \\
\hline $\mathrm{H}$ & -3.008126 & -1.034772 & -2.086851 \\
\hline $\mathrm{C}$ & -4.708747 & -1.785886 & -0.180587 \\
\hline $\mathrm{O}$ & -5.367156 & -1.5664 & -1.177956 \\
\hline $\mathrm{O}$ & -5.23508 & -2.550402 & 0.796015 \\
\hline $\mathrm{H}$ & -4.57514 & -2.734066 & 1.47897 \\
\hline $\mathrm{H}$ & -2.04069 & 1.950733 & 0.695696 \\
\hline $\mathrm{C}$ & -1.384716 & 3.728914 & -0.365858 \\
\hline $\mathrm{H}$ & -1.857423 & 3.325887 & 1.836746 \\
\hline $\mathrm{H}$ & -1.235864 & 4.791732 & -0.129728 \\
\hline $\mathrm{O}$ & -2.385013 & 3.576096 & -1.356202 \\
\hline $\mathrm{H}$ & -0.269259 & 2.148368 & -1.328192 \\
\hline $\mathrm{H}$ & 0.750107 & 3.610414 & -1.160492 \\
\hline $\mathrm{H}$ & -3.175102 & 4.044765 & -1.062364 \\
\hline $\mathrm{O}$ & 0.696934 & 1.491728 & 1.262733 \\
\hline
\end{tabular}




\begin{tabular}{|c|c|c|c|}
\hline $\mathrm{C}$ & 2.783667 & -2.631474 & -1.154109 \\
\hline $\mathrm{H}$ & 3.291866 & -3.397481 & -0.558106 \\
\hline $\mathrm{H}$ & 3.289581 & -2.540292 & -2.121631 \\
\hline $\mathrm{H}$ & 1.738769 & -2.919014 & -1.310457 \\
\hline $\mathrm{C}$ & 4.664967 & 0.18255 & 0.298101 \\
\hline $\mathrm{H}$ & 5.280251 & -0.46339 & 0.934227 \\
\hline $\mathrm{H}$ & 4.459237 & 1.124513 & 0.816386 \\
\hline $\mathrm{H}$ & 5.206014 & 0.383842 & -0.633279 \\
\hline \multicolumn{4}{|c|}{ 18) IM8: CBDO-TPA, G ${ }_{500 \mathrm{~K}}=-762.220200439 \mathrm{~kJ} / \mathrm{mol}$} \\
\hline $\mathrm{C}$ & -3.07528 & 0.20923 & 0.587846 \\
\hline $\mathrm{C}$ & -3.981679 & -1.001839 & 0.933653 \\
\hline $\mathrm{C}$ & -3.94188 & 0.511316 & -0.663436 \\
\hline $\mathrm{H}$ & -3.132581 & 0.99892 & 1.334416 \\
\hline $\mathrm{O}$ & -1.147229 & 2.220059 & -0.059996 \\
\hline $\mathrm{C}$ & -0.784845 & 1.049312 & 0.141451 \\
\hline $\mathrm{C}$ & 0.641073 & 0.604383 & 0.083605 \\
\hline $\mathrm{C}$ & 1.001438 & -0.736622 & 0.295468 \\
\hline $\mathrm{C}$ & 1.630045 & 1.568348 & -0.179381 \\
\hline $\mathrm{C}$ & 2.973946 & 1.192774 & -0.230828 \\
\hline $\mathrm{C}$ & 2.346381 & -1.115695 & 0.233266 \\
\hline $\mathrm{C}$ & 3.335766 & -0.152099 & -0.035963 \\
\hline $\mathrm{H}$ & 0.237666 & -1.471904 & 0.512106 \\
\hline $\mathrm{H}$ & 2.601245 & -2.151546 & 0.431999 \\
\hline $\mathrm{H}$ & 1.339779 & 2.600444 & -0.336945 \\
\hline $\mathrm{H}$ & 3.743554 & 1.930762 & -0.424319 \\
\hline $\mathrm{C}$ & 4.791471 & -0.498457 & -0.087777 \\
\hline $\mathrm{O}$ & 5.696565 & 0.309767 & 0.144382 \\
\hline $\mathrm{O}$ & 5.13135 & -1.788032 & -0.413816 \\
\hline $\mathrm{H}$ & 4.400977 & -2.349765 & -0.732664 \\
\hline $\mathrm{H}$ & -3.596704 & -1.93853 & 0.528935 \\
\hline $\mathrm{C}$ & -5.039117 & -0.384244 & -0.03583 \\
\hline $\mathrm{H}$ & -4.258337 & -1.120511 & 1.981384 \\
\hline $\mathrm{H}$ & -5.796599 & 0.195211 & 0.502158 \\
\hline $\mathrm{O}$ & -5.659102 & -1.263296 & -0.996316 \\
\hline $\mathrm{H}$ & -3.546718 & 0.041235 & -1.564702 \\
\hline $\mathrm{H}$ & -4.184494 & 1.556775 & -0.851172 \\
\hline $\mathrm{H}$ & -6.348898 & -1.805175 & -0.572681 \\
\hline $\mathrm{O}$ & -1.626933 & 0.025957 & 0.443126 \\
\hline
\end{tabular}




\begin{tabular}{|c|c|c|c|}
\hline $\mathrm{C}$ & -2.849145 & -0.100234 & 0.554654 \\
\hline $\mathrm{C}$ & -3.535853 & -1.389827 & 1.009164 \\
\hline $\mathrm{C}$ & -3.550605 & -0.206063 & -0.812064 \\
\hline $\mathrm{H}$ & -3.117781 & 0.778503 & 1.144304 \\
\hline $\mathrm{C}$ & -0.617239 & 0.736149 & 0.186385 \\
\hline $\mathrm{C}$ & 0.818815 & 0.421203 & 0.125127 \\
\hline $\mathrm{C}$ & 1.264812 & -0.872073 & 0.434547 \\
\hline $\mathrm{C}$ & 1.727723 & 1.424645 & -0.246154 \\
\hline $\mathrm{C}$ & 3.084411 & 1.12985 & -0.310049 \\
\hline $\mathrm{C}$ & 2.623549 & -1.162845 & 0.358638 \\
\hline $\mathrm{C}$ & 3.532465 & -0.165781 & -0.019925 \\
\hline $\mathrm{H}$ & 0.5593 & -1.641937 & 0.733517 \\
\hline $\mathrm{H}$ & 2.961201 & -2.162919 & 0.624839 \\
\hline $\mathrm{H}$ & 1.373258 & 2.424474 & -0.480502 \\
\hline $\mathrm{H}$ & 3.802254 & 1.896386 & -0.590182 \\
\hline $\mathrm{C}$ & 5.013767 & -0.429932 & -0.101699 \\
\hline $\mathrm{O}$ & 5.843377 & 0.427754 & 0.108719 \\
\hline $\mathrm{O}$ & 5.401103 & -1.674057 & -0.422064 \\
\hline $\mathrm{H}$ & 4.646292 & -2.225983 & -0.670937 \\
\hline $\mathrm{H}$ & -2.910569 & -2.258601 & 0.775262 \\
\hline $\mathrm{C}$ & -4.560956 & -1.157676 & -0.136892 \\
\hline $\mathrm{H}$ & -3.893187 & -1.436665 & 2.040417 \\
\hline $\mathrm{H}$ & -5.447334 & -0.61852 & 0.219868 \\
\hline $\mathrm{O}$ & -4.922188 & -2.256861 & -0.937937 \\
\hline $\mathrm{H}$ & -2.929615 & -0.769855 & -1.517536 \\
\hline $\mathrm{H}$ & -3.922948 & 0.711007 & -1.273245 \\
\hline $\mathrm{H}$ & -5.651465 & -2.722051 & -0.511146 \\
\hline $\mathrm{O}$ & -1.396441 & -0.22181 & 0.54002 \\
\hline $\mathrm{O}$ & -0.984151 & 1.914746 & -0.116882 \\
\hline $\mathrm{H}$ & -1.970018 & 2.239031 & -0.046118 \\
\hline \multicolumn{4}{|c|}{$\begin{array}{l}\text { 20) TS1a: Reduction of IM1 with CBDO, G500K }=-587.547516885 \mathrm{~kJ} / \mathrm{mol} \\
\qquad v=-678.71 \mathrm{~cm}^{-1}\end{array}$} \\
\hline $\mathrm{Sn}$ & 1.367391 & 0.151451 & -0.107017 \\
\hline $\mathrm{O}$ & -0.205068 & -1.321118 & 0.226252 \\
\hline $\mathrm{C}$ & -1.625168 & -1.313066 & 0.157194 \\
\hline $\mathrm{C}$ & -2.321343 & -0.491284 & -0.952438 \\
\hline $\mathrm{C}$ & -2.34343 & -0.460393 & 1.218969 \\
\hline $\mathrm{H}$ & -1.964173 & -2.354599 & 0.167493 \\
\hline $\mathrm{O}$ & 1.521987 & -1.186966 & -1.417699 \\
\hline
\end{tabular}




\begin{tabular}{|c|c|c|c|}
\hline $\mathrm{H}$ & -1.701248 & 0.356123 & -1.268318 \\
\hline $\mathrm{C}$ & -3.33809 & -0.026296 & 0.124139 \\
\hline $\mathrm{H}$ & -2.694362 & -1.027042 & -1.83012 \\
\hline $\mathrm{H}$ & -4.240676 & -0.651797 & 0.124066 \\
\hline $\mathrm{O}$ & -3.679453 & 1.344159 & 0.1764 \\
\hline $\mathrm{H}$ & -1.719989 & 0.394316 & 1.512567 \\
\hline $\mathrm{H}$ & -2.731937 & -0.959212 & 2.111175 \\
\hline $\mathrm{H}$ & -4.346465 & 1.51757 & -0.498419 \\
\hline $\mathrm{H}$ & 0.334074 & -1.632961 & -0.667908 \\
\hline $\mathrm{C}$ & 0.492072 & 2.031444 & -0.571784 \\
\hline $\mathrm{H}$ & -0.302635 & 2.258471 & 0.148672 \\
\hline $\mathrm{H}$ & 0.074922 & 2.01794 & -1.5838 \\
\hline $\mathrm{H}$ & 1.257702 & 2.8128 & -0.504547 \\
\hline $\mathrm{C}$ & 2.530311 & 0.066958 & 1.664824 \\
\hline $\mathrm{H}$ & 2.739099 & -0.978432 & 1.914385 \\
\hline $\mathrm{H}$ & 1.984201 & 0.541805 & 2.487693 \\
\hline $\mathrm{H}$ & 3.476099 & 0.599673 & 1.512847 \\
\hline \multicolumn{4}{|c|}{$\begin{array}{l}\text { 21) TS2a: Dehydration of IM2 with CBDO, G500K }=-1045.62735843 \mathrm{~kJ} / \mathrm{mol} \\
\qquad v=-649.73 \mathrm{~cm}^{-1}\end{array}$} \\
\hline $\mathrm{Sn}$ & -1.666843 & 0.131348 & 0.720151 \\
\hline $\mathrm{O}$ & -1.472659 & -1.042012 & -1.045227 \\
\hline $\mathrm{C}$ & -1.797653 & -0.790815 & 2.625127 \\
\hline $\mathrm{H}$ & -1.021773 & -0.405196 & 3.295348 \\
\hline $\mathrm{H}$ & -1.657112 & -1.870875 & 2.498897 \\
\hline $\mathrm{H}$ & -2.788443 & -0.615436 & 3.058479 \\
\hline $\mathrm{C}$ & -2.334703 & 2.086786 & 0.233438 \\
\hline $\mathrm{H}$ & -2.14229 & 2.27323 & -0.829852 \\
\hline $\mathrm{H}$ & -3.402595 & 2.219155 & 0.433667 \\
\hline $\mathrm{H}$ & -1.767194 & 2.816842 & 0.822723 \\
\hline $\mathrm{H}$ & -0.839081 & -1.771679 & -1.040566 \\
\hline $\mathrm{O}$ & -3.447907 & -1.036505 & 0.092208 \\
\hline $\mathrm{C}$ & -4.782508 & -0.75477 & -0.207247 \\
\hline $\mathrm{C}$ & -5.120711 & 0.287873 & -1.307915 \\
\hline $\mathrm{C}$ & -5.593277 & 0.093245 & 0.804308 \\
\hline $\mathrm{H}$ & -5.302589 & -1.702012 & -0.41155 \\
\hline $\mathrm{H}$ & -4.331383 & 1.04512 & -1.381429 \\
\hline $\mathrm{C}$ & -6.258277 & 0.79887 & -0.405069 \\
\hline $\mathrm{H}$ & -5.377695 & -0.077604 & -2.307448 \\
\hline $\mathrm{H}$ & -7.221314 & 0.3366 & -0.661635 \\
\hline
\end{tabular}




\begin{tabular}{|l|r|r|r|}
\hline $\mathrm{O}$ & -6.378799 & 2.210577 & -0.377375 \\
\hline $\mathrm{H}$ & -4.939808 & 0.794321 & 1.337947 \\
\hline $\mathrm{H}$ & -6.236048 & -0.430567 & 1.519622 \\
\hline $\mathrm{H}$ & -7.120825 & 2.438888 & 0.19509 \\
\hline $\mathrm{O}$ & -2.521304 & -1.333279 & -0.799545 \\
\hline $\mathrm{C}$ & 0.318306 & 0.244329 & 0.51751 \\
\hline $\mathrm{C}$ & 1.188487 & 1.112045 & 1.170981 \\
\hline $\mathrm{C}$ & 0.815922 & 1.783046 & 2.528495 \\
\hline $\mathrm{H}$ & 1.421648 & 2.532432 & 0.586455 \\
\hline $\mathrm{H}$ & 2.158897 & 0.605121 & 1.286476 \\
\hline $\mathrm{C}$ & -0.258585 & 2.0048 & 2.593267 \\
\hline $\mathrm{H}$ & 1.551786 & 3.043332 & 2.041515 \\
\hline $\mathrm{H}$ & 1.145162 & 1.307107 & 3.458112 \\
\hline $\mathrm{H}$ & 0.497115 & 2.915597 & 0.136346 \\
\hline $\mathrm{H}$ & 2.262049 & 2.682217 & -0.100141 \\
\hline $\mathrm{O}$ & 2.598999 & 3.053288 & 2.374252 \\
\hline $\mathrm{H}$ & 0.908354 & 4.255443 & 2.396392 \\
\hline $22 \mathrm{NS} \mathrm{D}$ & 1.434781 & 4.987377 & 2.053512 \\
\hline
\end{tabular}

22) TS2b: Dehydration of IM2 with TPA, G500K $=-1403.58025934 \mathrm{~kJ} / \mathrm{mol}$

\begin{tabular}{|l|r|r|r|}
\hline \multicolumn{5}{|c|}{$v=-107.91 \mathrm{~cm}^{-1}$} \\
\hline $\mathrm{Sn}$ & 1.321689 & 0.231584 & -0.05461 \\
\hline $\mathrm{C}$ & 3.086785 & 0.103418 & -0.860843 \\
\hline $\mathrm{C}$ & 4.271774 & 0.001326 & -0.111438 \\
\hline $\mathrm{C}$ & 5.573676 & -0.12331 & -0.933307 \\
\hline $\mathrm{H}$ & 4.608817 & -1.357473 & 0.560752 \\
\hline $\mathrm{H}$ & 4.351004 & 0.823224 & 0.61778 \\
\hline $\mathrm{C}$ & 5.360573 & -0.627115 & -1.884169 \\
\hline $\mathrm{H}$ & 6.066596 & -1.154776 & 0.112443 \\
\hline $\mathrm{H}$ & 6.170255 & 0.778602 & -1.105336 \\
\hline $\mathrm{O}$ & 6.665695 & -0.674231 & 0.898127 \\
\hline $\mathrm{H}$ & 6.702406 & -2.335203 & -0.342496 \\
\hline $\mathrm{H}$ & 4.153437 & -2.175577 & -0.012192 \\
\hline $\mathrm{H}$ & 4.406355 & -1.486105 & 1.629194 \\
\hline $\mathrm{C}$ & 7.616553 & -2.120697 & -0.562732 \\
\hline $\mathrm{H}$ & 1.332176 & -0.806184 & 1.778918 \\
\hline $\mathrm{H}$ & 1.561166 & -1.861784 & 1.597512 \\
\hline $\mathrm{H}$ & 0.360845 & -0.713741 & 2.271488 \\
\hline $\mathrm{O}$ & 2.10884 & -0.37581 & 2.42086 \\
\hline & 1.619731 & 2.246801 & 0.557853 \\
\hline
\end{tabular}




\begin{tabular}{|c|c|c|c|}
\hline $\mathrm{H}$ & 1.878722 & 2.900116 & -0.108316 \\
\hline $\mathrm{C}$ & 0.04744 & 0.140412 & -1.726458 \\
\hline $\mathrm{H}$ & -0.220832 & 1.159631 & -2.025877 \\
\hline $\mathrm{H}$ & 0.56875 & -0.35718 & -2.550276 \\
\hline $\mathrm{H}$ & -0.864453 & -0.409098 & -1.472323 \\
\hline $\mathrm{O}$ & -1.820756 & 3.22695 & -0.015718 \\
\hline $\mathrm{C}$ & -1.705027 & 2.074577 & 0.41664 \\
\hline $\mathrm{O}$ & -0.643447 & 1.611424 & 0.997368 \\
\hline $\mathrm{C}$ & -2.849503 & 1.08989 & 0.271989 \\
\hline $\mathrm{C}$ & -2.783024 & -0.178177 & 0.863603 \\
\hline $\mathrm{C}$ & -3.978815 & 1.445496 & -0.47716 \\
\hline $\mathrm{C}$ & -5.025761 & 0.54132 & -0.63938 \\
\hline $\mathrm{C}$ & -3.828879 & -1.08595 & 0.708777 \\
\hline $\mathrm{C}$ & -4.953742 & -0.72786 & -0.048167 \\
\hline $\mathrm{H}$ & -1.908826 & -0.449911 & 1.449657 \\
\hline $\mathrm{H}$ & -3.770884 & -2.067225 & 1.172143 \\
\hline $\mathrm{H}$ & -4.023122 & 2.4321 & -0.932508 \\
\hline $\mathrm{H}$ & -5.903845 & 0.808551 & -1.223171 \\
\hline $\mathrm{C}$ & -6.095786 & -1.668415 & -0.249194 \\
\hline $\mathrm{O}$ & -7.094341 & -1.411047 & -0.897545 \\
\hline $\mathrm{O}$ & -5.92273 & -2.852514 & 0.360505 \\
\hline $\mathrm{H}$ & -6.707953 & -3.391437 & 0.17543 \\
\hline $\mathrm{H}$ & 0.612426 & 2.364611 & 0.814114 \\
\hline \multicolumn{4}{|c|}{$\begin{array}{l}\text { 23) TS3a: Dehydration of IM3 with CBDO, G500K }=-1397.52611175 \mathrm{~kJ} / \mathrm{mol} \\
\qquad v=-1023.43 \mathrm{~cm}^{-1}\end{array}$} \\
\hline $\mathrm{Sn}$ & -1.497015 & -0.018608 & 0.365558 \\
\hline $\mathrm{O}$ & -1.401542 & -1.352275 & -1.233028 \\
\hline $\mathrm{C}$ & -1.317162 & -0.747962 & 2.338666 \\
\hline $\mathrm{H}$ & -0.48185 & -0.258197 & 2.849891 \\
\hline $\mathrm{H}$ & -1.135033 & -1.827661 & 2.297811 \\
\hline $\mathrm{H}$ & -2.250163 & -0.564853 & 2.882128 \\
\hline $\mathrm{C}$ & -2.030891 & 1.918009 & -0.275037 \\
\hline $\mathrm{H}$ & -2.004891 & 1.952357 & -1.370097 \\
\hline $\mathrm{H}$ & -3.032697 & 2.192385 & 0.070561 \\
\hline $\mathrm{H}$ & -1.30092 & 2.633629 & 0.119024 \\
\hline $\mathrm{O}$ & 0.567662 & 0.252253 & 0.059367 \\
\hline $\mathrm{C}$ & 1.390275 & -0.744036 & -0.05765 \\
\hline $\mathrm{O}$ & 1.04585 & -1.93145 & -0.155584 \\
\hline $\mathrm{C}$ & 2.850638 & -0.369123 & -0.073729 \\
\hline
\end{tabular}




\begin{tabular}{|c|c|c|c|}
\hline $\mathrm{C}$ & 3.8133 & -1.371004 & -0.240611 \\
\hline $\mathrm{C}$ & 3.250943 & 0.964937 & 0.08163 \\
\hline $\mathrm{C}$ & 4.604379 & 1.292633 & 0.071993 \\
\hline $\mathrm{C}$ & 5.168144 & -1.043989 & -0.264572 \\
\hline $\mathrm{C}$ & 5.568157 & 0.291713 & -0.111788 \\
\hline $\mathrm{H}$ & 3.493819 & -2.404749 & -0.348168 \\
\hline $\mathrm{H}$ & 5.898597 & -1.845368 & -0.369981 \\
\hline $\mathrm{H}$ & 2.497966 & 1.737884 & 0.211245 \\
\hline $\mathrm{H}$ & 4.924468 & 2.324249 & 0.199125 \\
\hline $\mathrm{C}$ & 7.012831 & 0.700689 & -0.115728 \\
\hline $\mathrm{O}$ & 7.424681 & 1.696757 & 0.442833 \\
\hline $\mathrm{O}$ & 7.876937 & -0.087768 & -0.780275 \\
\hline $\mathrm{H}$ & 7.407856 & -0.782486 & -1.262984 \\
\hline $\mathrm{H}$ & -0.688606 & -1.997911 & -1.059455 \\
\hline $\mathrm{O}$ & -3.30175 & -1.197171 & 0.014 \\
\hline $\mathrm{C}$ & -4.647602 & -0.911045 & -0.25681 \\
\hline $\mathrm{C}$ & -5.006499 & 0.037424 & -1.430904 \\
\hline $\mathrm{C}$ & -5.38335 & 0.047781 & 0.707736 \\
\hline $\mathrm{H}$ & -5.189312 & -1.861873 & -0.349101 \\
\hline $\mathrm{H}$ & -4.204764 & 0.765721 & -1.602759 \\
\hline $\mathrm{C}$ & -6.09039 & 0.658096 & -0.52979 \\
\hline $\mathrm{H}$ & -5.313802 & -0.40714 & -2.382526 \\
\hline $\mathrm{H}$ & -7.073169 & 0.197687 & -0.699521 \\
\hline $\mathrm{O}$ & -6.179183 & 2.067844 & -0.626334 \\
\hline $\mathrm{H}$ & -4.685283 & 0.778232 & 1.136637 \\
\hline $\mathrm{H}$ & -5.998941 & -0.386646 & 1.501876 \\
\hline $\mathrm{H}$ & -6.906563 & 2.364189 & -0.066431 \\
\hline $\mathrm{H}$ & -2.46382 & -1.609327 & -0.863442 \\
\hline
\end{tabular}

24) TS3b: Dehydration of IM3 with TPA, G500K $=-1713.34536049 \mathrm{~kJ} / \mathrm{mol}$

\begin{tabular}{|l|r|r|r|}
\hline \multicolumn{4}{|c|}{$v=-535.93 \mathrm{~cm}^{-1}$} \\
\hline $\mathrm{Sn}$ & 0.090075 & -0.152646 & -0.12346 \\
\hline $\mathrm{O}$ & -0.098103 & -2.192285 & 0.122681 \\
\hline $\mathrm{H}$ & 0.386901 & 0.393086 & -2.132766 \\
\hline $\mathrm{H}$ & -0.525196 & 0.854314 & -2.523629 \\
\hline $\mathrm{H}$ & 0.621932 & -0.507671 & -2.708272 \\
\hline $\mathrm{C}$ & 1.219246 & 1.100583 & -2.203174 \\
\hline $\mathrm{H}$ & 1.025884 & 0.54779 & 1.621368 \\
\hline $\mathrm{H}$ & 1.105982 & -0.279266 & 2.335092 \\
\hline
\end{tabular}




\begin{tabular}{|c|c|c|c|}
\hline $\mathrm{H}$ & 0.424305 & 1.349939 & 2.061122 \\
\hline $\mathrm{O}$ & -1.856825 & 0.235561 & 0.289624 \\
\hline $\mathrm{C}$ & -2.733664 & -0.405466 & -0.446627 \\
\hline $\mathrm{O}$ & -2.406532 & -1.254796 & -1.279009 \\
\hline $\mathrm{C}$ & -4.166296 & -0.036033 & -0.203206 \\
\hline $\mathrm{C}$ & -5.165618 & -0.667164 & -0.952664 \\
\hline $\mathrm{C}$ & -4.508552 & 0.928686 & 0.754018 \\
\hline $\mathrm{C}$ & -5.845678 & 1.257065 & 0.961054 \\
\hline $\mathrm{C}$ & -6.504705 & -0.346133 & -0.739156 \\
\hline $\mathrm{C}$ & -6.849036 & 0.614191 & 0.223166 \\
\hline $\mathrm{H}$ & -4.887832 & -1.403859 & -1.702412 \\
\hline $\mathrm{H}$ & -7.262183 & -0.829891 & -1.354306 \\
\hline $\mathrm{H}$ & -3.726653 & 1.41644 & 1.329975 \\
\hline $\mathrm{H}$ & -6.123285 & 2.007068 & 1.697824 \\
\hline $\mathrm{C}$ & -8.274399 & 1.008461 & 0.489689 \\
\hline $\mathrm{O}$ & -8.589375 & 2.088715 & 0.944326 \\
\hline $\mathrm{O}$ & -9.230822 & 0.106324 & 0.207712 \\
\hline $\mathrm{H}$ & -8.843653 & -0.741987 & -0.049557 \\
\hline $\mathrm{H}$ & -0.713314 & -2.594309 & -0.511689 \\
\hline $\mathrm{O}$ & 2.123227 & -1.747722 & -0.429615 \\
\hline $\mathrm{H}$ & 0.982753 & -2.337217 & -0.136497 \\
\hline $\mathrm{C}$ & 3.155723 & -1.948818 & 0.345929 \\
\hline $\mathrm{O}$ & 3.246428 & -2.869259 & 1.155798 \\
\hline $\mathrm{C}$ & 4.266691 & -0.936392 & 0.185738 \\
\hline $\mathrm{C}$ & 5.314341 & -0.914975 & 1.113254 \\
\hline $\mathrm{C}$ & 4.251062 & -0.010341 & -0.86619 \\
\hline $\mathrm{C}$ & 6.338301 & 0.02339 & 0.993324 \\
\hline $\mathrm{H}$ & 5.315173 & -1.63206 & 1.930726 \\
\hline $\mathrm{C}$ & 5.269318 & 0.931564 & -0.986128 \\
\hline $\mathrm{H}$ & 3.44387 & -0.035073 & -1.59362 \\
\hline $\mathrm{C}$ & 6.320873 & 0.949499 & -0.059763 \\
\hline $\mathrm{H}$ & 7.122854 & 0.038178 & 1.749016 \\
\hline $\mathrm{H}$ & 5.261776 & 1.655421 & -1.797703 \\
\hline $\mathrm{C}$ & 7.388208 & 1.991134 & -0.230091 \\
\hline $\mathrm{O}$ & 7.208447 & 3.038415 & -0.817414 \\
\hline $\mathrm{O}$ & 8.597137 & 1.731447 & 0.299569 \\
\hline $\mathrm{H}$ & 8.633932 & 0.829745 & 0.647991 \\
\hline \multicolumn{4}{|c|}{$\begin{array}{r}\text { 25) TS-IM4-IM5 exchange, } \mathrm{G}_{500 \mathrm{~K}}=-1530.23111673 \mathrm{~kJ} / \mathrm{m} \\
\qquad v=-507.35 \mathrm{~cm}^{-1}\end{array}$} \\
\hline
\end{tabular}




\begin{tabular}{|c|c|c|c|}
\hline $\mathrm{Sn}$ & -3.290557 & -0.555065 & -0.932838 \\
\hline $\mathrm{O}$ & -1.868808 & 0.705854 & -1.492887 \\
\hline $\mathrm{C}$ & -1.358532 & 1.69896 & -0.653884 \\
\hline $\mathrm{C}$ & -1.613002 & 3.178858 & -1.040021 \\
\hline $\mathrm{C}$ & 0.163149 & 1.981109 & -0.737278 \\
\hline $\mathrm{H}$ & -1.646108 & 1.540108 & 0.400106 \\
\hline $\mathrm{O}$ & -3.170254 & -3.343906 & 1.315482 \\
\hline $\mathrm{C}$ & -4.325907 & -3.188717 & 0.793043 \\
\hline $\mathrm{C}$ & -5.431314 & -4.083785 & 1.269447 \\
\hline $\mathrm{C}$ & -6.712754 & -3.946674 & 0.724005 \\
\hline $\mathrm{C}$ & -5.185655 & -5.053215 & 2.250552 \\
\hline $\mathrm{C}$ & -6.218049 & -5.880522 & 2.684167 \\
\hline $\mathrm{C}$ & -7.749209 & -4.767403 & 1.164459 \\
\hline $\mathrm{C}$ & -7.505866 & -5.734258 & 2.150469 \\
\hline $\mathrm{H}$ & -6.889914 & -3.199768 & -0.045578 \\
\hline $\mathrm{H}$ & -8.730942 & -4.660474 & 0.704816 \\
\hline $\mathrm{H}$ & -4.18768 & -5.155512 & 2.668958 \\
\hline $\mathrm{H}$ & -6.037546 & -6.639469 & 3.441744 \\
\hline $\mathrm{C}$ & -8.581499 & -6.652646 & 2.657215 \\
\hline $\mathrm{O}$ & -8.351257 & -7.739053 & 3.146821 \\
\hline $\mathrm{O}$ & -9.854238 & -6.229376 & 2.557679 \\
\hline $\mathrm{H}$ & -9.891183 & -5.314599 & 2.245303 \\
\hline $\mathrm{H}$ & -1.698451 & 3.265798 & -2.130491 \\
\hline $\mathrm{C}$ & -0.159604 & 3.478714 & -0.596451 \\
\hline $\mathrm{H}$ & -2.433887 & 3.714803 & -0.550214 \\
\hline $\mathrm{H}$ & -0.120102 & 3.80094 & 0.453552 \\
\hline $\mathrm{O}$ & 0.640606 & 4.325132 & -1.402868 \\
\hline $\mathrm{H}$ & 0.518086 & 1.771306 & -1.754181 \\
\hline $\mathrm{H}$ & 0.832113 & 1.519605 & -0.001772 \\
\hline $\mathrm{H}$ & 0.381099 & 5.238176 & -1.231772 \\
\hline $\mathrm{C}$ & -3.755389 & -1.374132 & -2.820074 \\
\hline $\mathrm{H}$ & -3.386621 & -0.715039 & -3.612676 \\
\hline $\mathrm{H}$ & -3.274148 & -2.354896 & -2.908103 \\
\hline $\mathrm{H}$ & -4.837996 & -1.504765 & -2.91935 \\
\hline $\mathrm{C}$ & -4.441387 & 0.622811 & 0.400875 \\
\hline $\mathrm{H}$ & -4.251927 & 1.688184 & 0.229965 \\
\hline $\mathrm{H}$ & -5.506308 & 0.411444 & 0.25822 \\
\hline $\mathrm{H}$ & -4.168495 & 0.367824 & 1.431809 \\
\hline
\end{tabular}




\begin{tabular}{|l|r|r|r|}
\hline $\mathrm{O}$ & -4.578782 & -2.33074 & -0.092568 \\
\hline $\mathrm{O}$ & -1.92516 & -1.712061 & 0.089039 \\
\hline $\mathrm{C}$ & -0.59225 & -1.373222 & 0.442615 \\
\hline $\mathrm{C}$ & 0.340863 & -2.540074 & 0.822993 \\
\hline $\mathrm{C}$ & -0.346581 & -0.750938 & 1.834977 \\
\hline $\mathrm{H}$ & -0.174914 & -0.760283 & -0.360943 \\
\hline $\mathrm{H}$ & -0.224607 & -3.355165 & 1.291233 \\
\hline $\mathrm{C}$ & 0.926141 & -1.613731 & 1.921711 \\
\hline $\mathrm{H}$ & 1.010772 & -2.935893 & 0.05388 \\
\hline $\mathrm{H}$ & 1.806353 & -1.067852 & 1.55552 \\
\hline $\mathrm{O}$ & 1.170595 & -2.155786 & 3.203492 \\
\hline $\mathrm{H}$ & -1.092101 & -1.118429 & 2.551214 \\
\hline $\mathrm{H}$ & -0.247808 & 0.336031 & 1.917024 \\
\hline $\mathrm{H}$ & 2.01224 & -2.626205 & 3.178134 \\
\hline $\mathrm{H}$ & -2.440559 & -2.530384 & 0.747102 \\
\hline
\end{tabular}

26) TS-IM5-IM6 exchange, $\mathrm{G}_{500 \mathrm{~K}}=-1860.8535961 \mathrm{~kJ} / \mathrm{mol}$

\begin{tabular}{|l|r|r|r|}
\hline \multicolumn{5}{|c|}{$v=-565.13 \mathrm{~cm}^{-1}$} \\
\hline $\mathrm{Sn}$ & -3.425442 & -0.446711 & -0.933397 \\
\hline $\mathrm{C}$ & -3.066716 & -3.504467 & 0.777129 \\
\hline $\mathrm{C}$ & -4.294191 & -3.196475 & 0.600357 \\
\hline $\mathrm{C}$ & -5.328585 & -4.146446 & 1.120492 \\
\hline $\mathrm{C}$ & -6.686159 & -3.865342 & 0.92957 \\
\hline $\mathrm{C}$ & -4.938269 & -5.314411 & 1.788747 \\
\hline $\mathrm{C}$ & -5.902878 & -6.197718 & 2.264862 \\
\hline $\mathrm{C}$ & -7.653093 & -4.742785 & 1.416832 \\
\hline $\mathrm{H}$ & -7.263391 & -5.909486 & 2.090158 \\
\hline $\mathrm{H}$ & -6.978067 & -2.963515 & 0.397554 \\
\hline $\mathrm{H}$ & -8.703526 & -4.519545 & 1.234503 \\
\hline $\mathrm{H}$ & -3.881404 & -5.5243 & 1.931276 \\
\hline $\mathrm{C}$ & -5.611392 & -7.110105 & 2.779739 \\
\hline $\mathrm{O}$ & -8.263206 & -6.895514 & 2.624853 \\
\hline $\mathrm{O}$ & -8.025228 & -8.078371 & 2.754465 \\
\hline $\mathrm{H}$ & -9.472426 & -6.421712 & 2.973908 \\
\hline $\mathrm{C}$ & -9.493135 & -5.455748 & 2.925682 \\
\hline $\mathrm{H}$ & -4.065208 & -1.239447 & -2.77616 \\
\hline $\mathrm{H}$ & -3.54713 & -0.735251 & -3.597686 \\
\hline $\mathrm{H}$ & -3.846571 & -2.312639 & -2.797126 \\
\hline & -5.145408 & -1.093594 & -2.882973 \\
\hline
\end{tabular}




\begin{tabular}{|c|c|c|c|}
\hline $\mathrm{C}$ & -4.17894 & 0.768196 & 0.616701 \\
\hline $\mathrm{H}$ & -3.630871 & 1.71514 & 0.646306 \\
\hline $\mathrm{H}$ & -5.2413 & 0.96939 & 0.441932 \\
\hline $\mathrm{H}$ & -4.067862 & 0.241282 & 1.570414 \\
\hline $\mathrm{O}$ & -4.668839 & -2.145662 & 0.014903 \\
\hline $\mathrm{O}$ & -1.920801 & -1.642414 & -0.199655 \\
\hline C & -0.802085 & -1.131559 & 0.523421 \\
\hline $\mathrm{C}$ & 0.183186 & -2.17266 & 1.089045 \\
\hline $\mathrm{C}$ & -1.041706 & -0.606413 & 1.95782 \\
\hline $\mathrm{H}$ & -0.303956 & -0.391003 & -0.107801 \\
\hline $\mathrm{H}$ & -0.333685 & -3.107823 & 1.336361 \\
\hline $\mathrm{C}$ & 0.283962 & -1.281778 & 2.35632 \\
\hline $\mathrm{H}$ & 1.092879 & -2.382614 & 0.518951 \\
\hline $\mathrm{H}$ & 1.128283 & -0.582769 & 2.288842 \\
\hline $\mathrm{O}$ & 0.251398 & -1.906165 & 3.623339 \\
\hline $\mathrm{H}$ & -1.879573 & -1.140226 & 2.424676 \\
\hline $\mathrm{H}$ & -1.150435 & 0.471852 & 2.10877 \\
\hline $\mathrm{H}$ & 1.13122 & -2.252828 & 3.813537 \\
\hline $\mathrm{H}$ & -2.406792 & -2.595972 & 0.309015 \\
\hline $\mathrm{O}$ & -1.826424 & 0.748656 & -1.581641 \\
\hline $\mathrm{C}$ & -2.323459 & 1.777986 & -2.198034 \\
\hline $\mathrm{C}$ & -1.334272 & 2.766486 & -2.744424 \\
\hline $\mathrm{O}$ & -3.546814 & 1.933064 & -2.328646 \\
\hline $\mathrm{C}$ & -1.797047 & 3.895532 & -3.428795 \\
\hline $\mathrm{C}$ & 0.042026 & 2.56174 & -2.579154 \\
\hline $\mathrm{C}$ & -0.890063 & 4.82216 & -3.940117 \\
\hline $\mathrm{H}$ & -2.866506 & 4.039602 & -3.561759 \\
\hline $\mathrm{C}$ & 0.9495 & 3.480843 & -3.098749 \\
\hline $\mathrm{H}$ & 0.393174 & 1.681196 & -2.047313 \\
\hline $\mathrm{C}$ & 0.487815 & 4.618893 & -3.774506 \\
\hline $\mathrm{H}$ & -1.277251 & 5.678401 & -4.491112 \\
\hline $\mathrm{H}$ & 2.019756 & 3.328499 & -2.981299 \\
\hline $\mathrm{C}$ & 1.513788 & 5.571983 & -4.318193 \\
\hline $\mathrm{O}$ & 2.660448 & 5.249449 & -4.552251 \\
\hline $\mathrm{O}$ & 1.126287 & 6.838933 & -4.548239 \\
\hline $\mathrm{H}$ & 0.224155 & 6.987411 & -4.232995 \\
\hline
\end{tabular}




\begin{tabular}{|c|c|c|c|}
\hline $\mathrm{Sn}$ & 0.460367 & -0.699052 & -1.265515 \\
\hline $\mathrm{O}$ & -0.209325 & 0.86696 & 0.122961 \\
\hline $\mathrm{C}$ & -2.371309 & -1.992031 & -0.153008 \\
\hline $\mathrm{C}$ & -3.774522 & -1.787667 & -0.764151 \\
\hline $\mathrm{C}$ & -3.032435 & -2.403332 & 1.181095 \\
\hline $\mathrm{H}$ & -1.879449 & -2.858161 & -0.609666 \\
\hline $\mathrm{O}$ & -1.095931 & -0.093315 & 2.039103 \\
\hline $\mathrm{C}$ & -1.370375 & 0.266839 & 0.88508 \\
\hline $\mathrm{C}$ & -2.607967 & 1.107675 & 0.596673 \\
\hline $\mathrm{C}$ & -2.807416 & 1.736545 & -0.63732 \\
\hline $\mathrm{C}$ & -3.579213 & 1.218391 & 1.59384 \\
\hline $\mathrm{C}$ & -4.75349 & 1.930493 & 1.356605 \\
\hline $\mathrm{C}$ & -3.979839 & 2.44614 & -0.880467 \\
\hline $\mathrm{C}$ & -4.967529 & 2.537069 & 0.112835 \\
\hline $\mathrm{H}$ & -2.049098 & 1.674728 & -1.413028 \\
\hline $\mathrm{H}$ & -4.093597 & 2.946472 & -1.841221 \\
\hline $\mathrm{H}$ & -3.40825 & 0.740533 & 2.555593 \\
\hline $\mathrm{H}$ & -5.514102 & 2.013821 & 2.129241 \\
\hline $\mathrm{C}$ & -6.252031 & 3.283124 & -0.092128 \\
\hline $\mathrm{O}$ & -6.923204 & 3.719672 & 0.821059 \\
\hline $\mathrm{O}$ & -6.6705 & 3.466119 & -1.358713 \\
\hline $\mathrm{H}$ & -6.107476 & 2.986772 & -1.982031 \\
\hline $\mathrm{H}$ & -4.128405 & -0.763046 & -0.607876 \\
\hline $\mathrm{C}$ & -4.309123 & -2.700082 & 0.371188 \\
\hline $\mathrm{H}$ & -3.930575 & -2.090523 & -1.804285 \\
\hline $\mathrm{H}$ & -4.357432 & -3.751226 & 0.05553 \\
\hline $\mathrm{O}$ & -5.500904 & -2.322383 & 1.031552 \\
\hline $\mathrm{H}$ & -3.204544 & -1.538092 & 1.82699 \\
\hline $\mathrm{H}$ & -2.570787 & -3.211812 & 1.754786 \\
\hline $\mathrm{H}$ & -6.248201 & -2.575404 & 0.476752 \\
\hline $\mathrm{C}$ & -0.283182 & 0.101778 & -3.070714 \\
\hline $\mathrm{H}$ & -1.351528 & -0.119665 & -3.169433 \\
\hline $\mathrm{H}$ & -0.133296 & 1.187374 & -3.075747 \\
\hline $\mathrm{H}$ & 0.252881 & -0.332714 & -3.921639 \\
\hline $\mathrm{C}$ & 0.916246 & -2.757328 & -0.994296 \\
\hline $\mathrm{H}$ & 0.798141 & -3.020089 & 0.062969 \\
\hline $\mathrm{H}$ & 0.243162 & -3.378899 & -1.595152 \\
\hline $\mathrm{H}$ & 1.949214 & -2.955214 & -1.300618 \\
\hline $\mathrm{O}$ & -1.427544 & -0.935374 & -0.20231 \\
\hline
\end{tabular}




\begin{tabular}{|c|c|c|c|}
\hline $\mathrm{H}$ & 0.459381 & 1.113639 & 0.782019 \\
\hline $\mathrm{O}$ & 2.176473 & 0.200822 & -0.921044 \\
\hline $\mathrm{C}$ & 3.339049 & -0.463794 & -0.509548 \\
\hline $\mathrm{C}$ & 3.399543 & -1.059548 & 0.92369 \\
\hline $\mathrm{C}$ & 4.534722 & 0.444518 & -0.140848 \\
\hline $\mathrm{H}$ & 3.650983 & -1.218563 & -1.249155 \\
\hline $\mathrm{H}$ & 2.749602 & -0.48389 & 1.595432 \\
\hline $\mathrm{C}$ & 4.850263 & -0.524393 & 1.012081 \\
\hline $\mathrm{H}$ & 3.226738 & -2.133207 & 1.056175 \\
\hline $\mathrm{H}$ & 4.160541 & 1.392574 & 0.265677 \\
\hline $\mathrm{H}$ & 5.305702 & 0.632617 & -0.894941 \\
\hline $\mathrm{H}$ & 5.577162 & -1.28993 & 0.707218 \\
\hline $\mathrm{O}$ & 5.271285 & 0.109749 & 2.206007 \\
\hline $\mathrm{H}$ & 5.467649 & -0.573343 & 2.858077 \\
\hline \multicolumn{4}{|c|}{$\begin{aligned} & \text { 28) TS5a: Box Transfer, G500K }=-916.383864528 \mathrm{~kJ} / \mathrm{mol} \\
& v=-283.88 \mathrm{~cm}^{-1}\end{aligned}$} \\
\hline $\mathrm{Sn}$ & -1.550503 & -1.697908 & -0.291145 \\
\hline $\mathrm{O}$ & -1.15906 & 0.380857 & 0.357077 \\
\hline $\mathrm{C}$ & -2.147145 & 1.389779 & 0.661352 \\
\hline $\mathrm{C}$ & -3.034446 & 1.751536 & -0.540301 \\
\hline $\mathrm{C}$ & -1.643996 & 2.841681 & 0.718967 \\
\hline $\mathrm{H}$ & -2.707223 & 1.08627 & 1.549338 \\
\hline $\mathrm{O}$ & -0.330032 & -1.91916 & 1.094287 \\
\hline $\mathrm{C}$ & -0.158828 & 0.14653 & 1.402803 \\
\hline $\mathrm{C}$ & 1.232404 & 0.192262 & 0.845086 \\
\hline $\mathrm{C}$ & 2.291124 & -0.119515 & 1.706489 \\
\hline $\mathrm{C}$ & 1.496057 & 0.594174 & -0.469245 \\
\hline $\mathrm{C}$ & 2.81081 & 0.661004 & -0.925255 \\
\hline $\mathrm{C}$ & 3.60288 & -0.065888 & 1.246447 \\
\hline $\mathrm{C}$ & 3.870528 & 0.317668 & -0.077626 \\
\hline $\mathrm{H}$ & 2.079779 & -0.405966 & 2.733632 \\
\hline $\mathrm{H}$ & 4.407109 & -0.294031 & 1.944897 \\
\hline $\mathrm{H}$ & 0.681821 & 0.864349 & -1.136172 \\
\hline $\mathrm{H}$ & 3.02313 & 0.976477 & -1.94409 \\
\hline $\mathrm{C}$ & 5.263448 & 0.401928 & -0.626469 \\
\hline $\mathrm{O}$ & 5.565209 & 1.093483 & -1.578169 \\
\hline $\mathrm{O}$ & 6.213184 & -0.336799 & -0.023441 \\
\hline $\mathrm{H}$ & 5.825652 & -0.916103 & 0.647269 \\
\hline $\mathrm{H}$ & -2.491396 & 1.59606 & -1.480497 \\
\hline
\end{tabular}




\begin{tabular}{|c|c|c|c|}
\hline $\mathrm{C}$ & -2.897143 & 3.227484 & -0.112724 \\
\hline $\mathrm{H}$ & -4.03365 & 1.31201 & -0.598218 \\
\hline $\mathrm{H}$ & -3.727278 & 3.537412 & 0.535011 \\
\hline $\mathrm{O}$ & -2.740305 & 4.120141 & -1.193113 \\
\hline $\mathrm{H}$ & -0.740412 & 2.958397 & 0.108965 \\
\hline $\mathrm{H}$ & -1.503558 & 3.294951 & 1.703459 \\
\hline $\mathrm{H}$ & -2.759113 & 5.021323 & -0.849752 \\
\hline $\mathrm{C}$ & -0.877453 & -1.68192 & -2.302473 \\
\hline $\mathrm{H}$ & 0.209101 & -1.548487 & -2.324818 \\
\hline $\mathrm{H}$ & -1.138041 & -2.627883 & -2.790428 \\
\hline $\mathrm{H}$ & -1.361 & -0.860818 & -2.844489 \\
\hline $\mathrm{C}$ & -3.641019 & -1.864817 & 0.034417 \\
\hline $\mathrm{H}$ & -3.915292 & -2.923226 & 0.110823 \\
\hline $\mathrm{H}$ & -3.906483 & -1.362871 & 0.971415 \\
\hline $\mathrm{H}$ & -4.191663 & -1.413436 & -0.797951 \\
\hline $\mathrm{O}$ & -0.452701 & 0.39267 & 2.549616 \\
\hline \multicolumn{4}{|c|}{$\begin{array}{l}\text { 29) TS5+: Box Transfer with } \mathrm{H}_{3} \mathrm{O}^{+}, \mathrm{G}_{500 \mathrm{~K}}=-830.515632478 \mathrm{~kJ} / \mathrm{mol} \\
\qquad v=-246.37 \mathrm{~cm}^{-1}\end{array}$} \\
\hline $\mathrm{Sn}$ & 2.524075 & -0.612667 & -1.034055 \\
\hline $\mathrm{C}$ & 1.282604 & 1.71122 & 0.761736 \\
\hline $\mathrm{C}$ & 0.027662 & 2.578907 & 0.962727 \\
\hline $\mathrm{C}$ & 1.979625 & 2.894407 & 0.068781 \\
\hline $\mathrm{H}$ & 1.75884 & 1.415448 & 1.701466 \\
\hline $\mathrm{O}$ & 0.934181 & -1.720233 & -0.517577 \\
\hline $\mathrm{C}$ & 0.259846 & -0.644849 & 0.647665 \\
\hline $\mathrm{C}$ & -1.168352 & -0.455554 & 0.215061 \\
\hline $\mathrm{C}$ & -2.151046 & -0.547082 & 1.201933 \\
\hline $\mathrm{C}$ & -1.522345 & -0.172212 & -1.109459 \\
\hline $\mathrm{C}$ & -2.857577 & 0.020284 & -1.442184 \\
\hline $\mathrm{C}$ & -3.491027 & -0.341917 & 0.871173 \\
\hline $\mathrm{C}$ & -3.847664 & -0.051734 & -0.451023 \\
\hline $\mathrm{H}$ & -1.865799 & -0.775222 & 2.225749 \\
\hline $\mathrm{H}$ & -4.241875 & -0.441854 & 1.653721 \\
\hline $\mathrm{H}$ & -0.762111 & -0.100017 & -1.881484 \\
\hline $\mathrm{H}$ & -3.14389 & 0.234548 & -2.468896 \\
\hline $\mathrm{C}$ & -5.273641 & 0.166714 & -0.870606 \\
\hline $\mathrm{O}$ & -5.66597 & -0.023082 & -2.003109 \\
\hline $\mathrm{O}$ & -6.137534 & 0.595168 & 0.065823 \\
\hline $\mathrm{H}$ & -5.679832 & 0.802786 & 0.892333 \\
\hline
\end{tabular}




\begin{tabular}{|c|c|c|c|}
\hline $\mathrm{H}$ & -0.655889 & 2.471983 & 0.112847 \\
\hline $\mathrm{C}$ & 0.943982 & 3.813961 & 0.749519 \\
\hline $\mathrm{H}$ & -0.515067 & 2.478995 & 1.90647 \\
\hline $\mathrm{H}$ & 1.32109 & 4.206113 & 1.702759 \\
\hline $\mathrm{O}$ & 0.481413 & 4.856671 & -0.0781 \\
\hline $\mathrm{H}$ & 1.787136 & 2.874879 & -1.011108 \\
\hline $\mathrm{H}$ & 3.042127 & 3.057673 & 0.267756 \\
\hline $\mathrm{H}$ & -0.065381 & 5.448617 & 0.451981 \\
\hline $\mathrm{C}$ & 2.415943 & -0.130323 & -3.068812 \\
\hline $\mathrm{H}$ & 1.364603 & -0.094036 & -3.368426 \\
\hline $\mathrm{H}$ & 2.885283 & 0.845926 & -3.227103 \\
\hline $\mathrm{H}$ & 2.946714 & -0.891269 & -3.649312 \\
\hline $\mathrm{C}$ & 4.147859 & -0.91865 & 0.257623 \\
\hline $\mathrm{H}$ & 4.721857 & -1.787767 & -0.077786 \\
\hline $\mathrm{H}$ & 4.78767 & -0.030543 & 0.247968 \\
\hline $\mathrm{H}$ & 3.751312 & -1.089971 & 1.263264 \\
\hline $\mathrm{O}$ & 1.121991 & 0.539591 & -0.059034 \\
\hline $\mathrm{O}$ & 0.617146 & -0.915658 & 1.774454 \\
\hline $\mathrm{H}$ & 1.047633 & -3.512822 & 1.857714 \\
\hline $\mathrm{O}$ & 1.257267 & -3.855986 & 0.977582 \\
\hline $\mathrm{H}$ & 1.050152 & -2.584104 & -0.021088 \\
\hline $\mathrm{H}$ & 0.645878 & -4.591413 & 0.842312 \\
\hline \multicolumn{4}{|c|}{$\begin{array}{l}\text { 30) TS5b.1: Coordinate Transfer Step 1, G500K }=-943.412504543 \mathrm{~kJ} / \mathrm{mol} \\
\qquad v=-279.64 \mathrm{~cm}^{-1}\end{array}$} \\
\hline $\mathrm{Sn}$ & 2.846617 & -0.515487 & -0.040557 \\
\hline $\mathrm{O}$ & 1.190823 & -1.712373 & 0.624197 \\
\hline $\mathrm{C}$ & 0.679635 & 1.87014 & 0.765955 \\
\hline $\mathrm{C}$ & -0.714169 & 2.340207 & 1.245398 \\
\hline $\mathrm{C}$ & 0.365921 & 2.476779 & -0.630771 \\
\hline $\mathrm{H}$ & 1.464702 & 2.461738 & 1.257501 \\
\hline $\mathrm{O}$ & 1.168124 & -0.489041 & -1.222019 \\
\hline $\mathrm{C}$ & 0.521438 & -0.827382 & -0.087213 \\
\hline $\mathrm{C}$ & -0.973992 & -0.865689 & -0.098071 \\
\hline $\mathrm{C}$ & -1.65621 & -1.278225 & 1.051252 \\
\hline $\mathrm{C}$ & -1.684059 & -0.492843 & -1.242172 \\
\hline $\mathrm{C}$ & -3.077074 & -0.517509 & -1.234134 \\
\hline $\mathrm{C}$ & -3.048192 & -1.28889 & 1.066092 \\
\hline $\mathrm{C}$ & -3.765989 & -0.900236 & -0.076332 \\
\hline $\mathrm{H}$ & -1.092097 & -1.582088 & 1.929317 \\
\hline
\end{tabular}




\begin{tabular}{|c|c|c|c|}
\hline $\mathrm{H}$ & -3.556212 & -1.634817 & 1.965298 \\
\hline $\mathrm{H}$ & -1.143731 & -0.189215 & -2.135216 \\
\hline $\mathrm{H}$ & -3.639122 & -0.233287 & -2.120519 \\
\hline $\mathrm{C}$ & -5.265653 & -0.902529 & -0.122753 \\
\hline $\mathrm{O}$ & -5.900678 & -0.995155 & -1.153542 \\
\hline $\mathrm{O}$ & -5.922959 & -0.791949 & 1.046524 \\
\hline $\mathrm{H}$ & -5.307615 & -0.621882 & 1.773088 \\
\hline $\mathrm{H}$ & -1.466214 & 1.561194 & 1.074596 \\
\hline $\mathrm{C}$ & -0.711409 & 3.33316 & 0.056894 \\
\hline $\mathrm{H}$ & -0.8048 & 2.738096 & 2.261581 \\
\hline $\mathrm{H}$ & -0.31913 & 4.315489 & 0.355144 \\
\hline $\mathrm{O}$ & -1.897189 & 3.482715 & -0.702616 \\
\hline $\mathrm{H}$ & -0.109807 & 1.740572 & -1.28442 \\
\hline $\mathrm{H}$ & 1.169342 & 2.98751 & -1.171944 \\
\hline $\mathrm{H}$ & -2.50632 & 4.039783 & -0.203747 \\
\hline $\mathrm{C}$ & 3.898248 & 1.330308 & 0.018251 \\
\hline $\mathrm{H}$ & 3.331108 & 2.097502 & -0.518929 \\
\hline $\mathrm{H}$ & 4.019357 & 1.642162 & 1.062299 \\
\hline $\mathrm{H}$ & 4.885132 & 1.202358 & -0.439004 \\
\hline $\mathrm{C}$ & 4.151232 & -2.181167 & -0.24363 \\
\hline $\mathrm{H}$ & 5.108804 & -1.874488 & -0.677356 \\
\hline $\mathrm{H}$ & 4.324951 & -2.613169 & 0.749688 \\
\hline $\mathrm{H}$ & 3.671766 & -2.93615 & -0.875918 \\
\hline $\mathrm{O}$ & 1.026965 & 0.517839 & 0.93418 \\
\hline \multicolumn{4}{|c|}{$\begin{array}{l}\text { 31) TS5b.2: Coordinated Transfer Step 2, G500K }=-943.763960544 \mathrm{~kJ} / \mathrm{mol} \\
\qquad v=-294.64 \mathrm{~cm}^{-1}\end{array}$} \\
\hline $\mathrm{Sn}$ & 1.111567 & -2.047005 & 0.242433 \\
\hline $\mathrm{O}$ & 0.471587 & -0.867998 & 1.544476 \\
\hline $\mathrm{C}$ & 2.356995 & 1.569843 & 0.361342 \\
\hline $\mathrm{C}$ & 2.953166 & 2.887538 & 0.868804 \\
\hline $\mathrm{C}$ & 3.142858 & 1.728728 & -0.952864 \\
\hline $\mathrm{H}$ & 2.655647 & 0.698339 & 0.952123 \\
\hline $\mathrm{O}$ & 0.921462 & -0.290779 & -0.952075 \\
\hline $\mathrm{C}$ & 0.317055 & 0.522531 & -0.167599 \\
\hline $\mathrm{C}$ & -1.170651 & 0.612678 & -0.123234 \\
\hline $\mathrm{C}$ & -1.810036 & 1.433867 & 0.812975 \\
\hline $\mathrm{C}$ & -1.92183 & -0.128096 & -1.041914 \\
\hline $\mathrm{C}$ & -3.312317 & -0.058482 & -1.017758 \\
\hline $\mathrm{C}$ & -3.199207 & 1.509452 & 0.830441 \\
\hline
\end{tabular}




\begin{tabular}{|c|c|c|c|}
\hline $\mathrm{C}$ & -3.957617 & 0.765201 & -0.086999 \\
\hline $\mathrm{H}$ & -1.2226 & 1.992981 & 1.535813 \\
\hline $\mathrm{H}$ & -3.674663 & 2.12755 & 1.59069 \\
\hline $\mathrm{H}$ & -1.41654 & -0.750573 & -1.776645 \\
\hline $\mathrm{H}$ & -3.906098 & -0.634151 & -1.723618 \\
\hline $\mathrm{C}$ & -5.458824 & 0.798263 & -0.106052 \\
\hline $\mathrm{O}$ & -6.136213 & -0.096448 & -0.568947 \\
\hline $\mathrm{O}$ & -6.064066 & 1.879804 & 0.416491 \\
\hline $\mathrm{H}$ & -5.417908 & 2.558898 & 0.654758 \\
\hline $\mathrm{H}$ & 2.304814 & 3.728282 & 0.595654 \\
\hline $\mathrm{C}$ & 4.063251 & 2.712652 & -0.203958 \\
\hline $\mathrm{H}$ & 3.238344 & 2.950232 & 1.922495 \\
\hline $\mathrm{H}$ & 4.944465 & 2.205897 & 0.211202 \\
\hline $\mathrm{O}$ & 4.44473 & 3.838066 & -0.965974 \\
\hline $\mathrm{H}$ & 2.544018 & 2.269489 & -1.695024 \\
\hline $\mathrm{H}$ & 3.580284 & 0.832055 & -1.398446 \\
\hline $\mathrm{H}$ & 5.082539 & 4.350308 & -0.454974 \\
\hline $\mathrm{C}$ & -0.20268 & -3.453934 & -0.657845 \\
\hline $\mathrm{H}$ & -1.242309 & -3.144682 & -0.505181 \\
\hline $\mathrm{H}$ & 0.005593 & -3.521087 & -1.732219 \\
\hline $\mathrm{H}$ & -0.05239 & -4.442732 & -0.208872 \\
\hline $\mathrm{C}$ & 3.183227 & -2.490046 & 0.0748 \\
\hline $\mathrm{H}$ & 3.778352 & -1.652852 & 0.455349 \\
\hline $\mathrm{H}$ & 3.421275 & -3.392058 & 0.650684 \\
\hline $\mathrm{H}$ & 3.435835 & -2.667648 & -0.977129 \\
\hline $\mathrm{O}$ & 0.920328 & 1.584962 & 0.306135 \\
\hline \multicolumn{4}{|c|}{$\begin{aligned} \text { 32) TS5c: Acid Transfer of IM5, } \mathrm{G}_{500 \mathrm{~K}} & =-1697.21031298 \mathrm{~kJ} / \mathrm{mol} \\
v & =-176.25 \mathrm{~cm}^{-1}\end{aligned}$} \\
\hline $\mathrm{Sn}$ & 0.293414 & -1.297308 & -0.310752 \\
\hline $\mathrm{O}$ & -0.391666 & 0.233929 & 1.103357 \\
\hline $\mathrm{C}$ & -2.800888 & -2.213955 & -0.057106 \\
\hline $\mathrm{C}$ & -3.92366 & -1.704241 & -0.992205 \\
\hline $\mathrm{C}$ & -3.873988 & -2.581492 & 0.993733 \\
\hline $\mathrm{H}$ & -2.351229 & -3.12873 & -0.467302 \\
\hline $\mathrm{O}$ & -1.896422 & -0.547327 & 2.666247 \\
\hline $\mathrm{C}$ & -1.778911 & -0.102492 & 1.530701 \\
\hline $\mathrm{C}$ & -2.752597 & 0.89211 & 0.935465 \\
\hline $\mathrm{C}$ & -2.46394 & 1.620213 & -0.223838 \\
\hline $\mathrm{C}$ & -3.986637 & 1.056632 & 1.569614 \\
\hline
\end{tabular}




\begin{tabular}{|c|c|c|c|}
\hline $\mathrm{C}$ & -4.940188 & 1.920729 & 1.035922 \\
\hline $\mathrm{C}$ & -3.417006 & 2.479056 & -0.764357 \\
\hline $\mathrm{C}$ & -4.667191 & 2.623714 & -0.143953 \\
\hline $\mathrm{H}$ & -1.496975 & 1.525432 & -0.709254 \\
\hline $\mathrm{H}$ & -3.156262 & 3.051269 & -1.654005 \\
\hline $\mathrm{H}$ & -4.19415 & 0.503879 & 2.482716 \\
\hline $\mathrm{H}$ & -5.903148 & 2.048912 & 1.524381 \\
\hline $\mathrm{C}$ & -5.729092 & 3.535706 & -0.683124 \\
\hline $\mathrm{O}$ & -6.596574 & 4.030896 & 0.006884 \\
\hline $\mathrm{O}$ & -5.703653 & 3.802619 & -2.002098 \\
\hline $\mathrm{H}$ & -5.035661 & 3.265157 & -2.449918 \\
\hline $\mathrm{H}$ & -4.1195 & -0.639623 & -0.823921 \\
\hline $\mathrm{C}$ & -4.906194 & -2.557812 & -0.149708 \\
\hline $\mathrm{H}$ & -3.833171 & -1.909121 & -2.063854 \\
\hline $\mathrm{H}$ & -5.049949 & -3.555197 & -0.587549 \\
\hline $\mathrm{O}$ & -6.149934 & -1.991137 & 0.215173 \\
\hline $\mathrm{H}$ & -4.063278 & -1.746978 & 1.675846 \\
\hline $\mathrm{H}$ & -3.741867 & -3.506065 & 1.563131 \\
\hline $\mathrm{H}$ & -6.745314 & -2.053255 & -0.541095 \\
\hline $\mathrm{C}$ & -0.097371 & -0.568674 & -2.251588 \\
\hline $\mathrm{H}$ & -1.175423 & -0.430929 & -2.383056 \\
\hline $\mathrm{H}$ & 0.419731 & 0.387676 & -2.387936 \\
\hline $\mathrm{H}$ & 0.278553 & -1.280705 & -2.993215 \\
\hline $\mathrm{C}$ & 0.706837 & -3.180964 & 0.544065 \\
\hline $\mathrm{H}$ & 0.372115 & -3.160393 & 1.587452 \\
\hline $\mathrm{H}$ & 0.151254 & -3.960995 & 0.011671 \\
\hline $\mathrm{H}$ & 1.777886 & -3.39631 & 0.495172 \\
\hline $\mathrm{O}$ & -1.745289 & -1.352954 & 0.295961 \\
\hline $\mathrm{O}$ & 2.021182 & -0.253625 & 0.227689 \\
\hline $\mathrm{C}$ & 2.996365 & -0.683402 & -0.521603 \\
\hline $\mathrm{C}$ & 4.339376 & -0.055378 & -0.295643 \\
\hline $\mathrm{C}$ & 4.508477 & 0.935526 & 0.678304 \\
\hline $\mathrm{C}$ & 5.4273 & -0.464688 & -1.077509 \\
\hline $\mathrm{C}$ & 6.678214 & 0.115292 & -0.888132 \\
\hline $\mathrm{C}$ & 5.76386 & 1.508375 & 0.87771 \\
\hline $\mathrm{C}$ & 6.853782 & 1.097085 & 0.097444 \\
\hline $\mathrm{H}$ & 3.657584 & 1.258524 & 1.272195 \\
\hline $\mathrm{H}$ & 5.86496 & 2.295843 & 1.623693 \\
\hline $\mathrm{H}$ & 5.282216 & -1.233986 & -1.832388 \\
\hline
\end{tabular}




\begin{tabular}{|l|r|r|r|}
\hline $\mathrm{H}$ & 7.527831 & -0.190034 & -1.494424 \\
\hline $\mathrm{C}$ & 8.224679 & 1.690287 & 0.25744 \\
\hline $\mathrm{O}$ & 9.046328 & 1.712514 & -0.635671 \\
\hline $\mathrm{O}$ & 8.538482 & 2.214141 & 1.455631 \\
\hline $\mathrm{H}$ & 7.842437 & 2.038872 & 2.104018 \\
\hline $\mathrm{O}$ & 2.81754 & -1.561618 & -1.376252 \\
\hline $\mathrm{H}$ & 0.130506 & 0.364052 & 1.911202 \\
\hline
\end{tabular}

33) TS5c+: Acid Transfer of IM5- $\mathrm{H}_{3} \mathrm{O}^{+}$with TPA, G500K $=-1494.47459286 \mathrm{~kJ} / \mathrm{mol}$ $v=-243.60 \mathrm{~cm}^{-1}$

\begin{tabular}{|l|r|r|r|}
\hline Sn & 0.313561 & -1.9301 & -1.74819 \\
\hline $\mathrm{O}$ & -0.20474 & 0.924851 & -0.572603 \\
\hline $\mathrm{C}$ & -1.982871 & -2.372628 & 0.12594 \\
\hline $\mathrm{C}$ & -3.075515 & -3.01145 & -0.755652 \\
\hline $\mathrm{C}$ & -3.059589 & -2.082162 & 1.197584 \\
\hline $\mathrm{H}$ & -1.289372 & -3.126232 & 0.51657 \\
\hline $\mathrm{C}$ & -1.36015 & 0.269892 & 0.386884 \\
\hline $\mathrm{C}$ & -2.632483 & 0.858994 & -0.15294 \\
\hline $\mathrm{C}$ & -2.984654 & 0.777573 & -1.501358 \\
\hline $\mathrm{C}$ & -3.447772 & 1.544552 & 0.753851 \\
\hline $\mathrm{C}$ & -4.625982 & 2.138966 & 0.309714 \\
\hline $\mathrm{C}$ & -4.169212 & 1.36008 & -1.942433 \\
\hline $\mathrm{H}$ & -4.997127 & 2.037611 & -1.03663 \\
\hline $\mathrm{H}$ & -2.344015 & 0.255701 & -2.204499 \\
\hline $\mathrm{H}$ & -4.417407 & 1.30018 & -3.00076 \\
\hline $\mathrm{H}$ & -3.159854 & 1.603042 & 1.800501 \\
\hline $\mathrm{C}$ & -5.266509 & 2.677857 & 1.003438 \\
\hline $\mathrm{O}$ & -6.277985 & 2.697525 & -1.46457 \\
\hline $\mathrm{O}$ & -6.739606 & 3.668676 & -0.903592 \\
\hline $\mathrm{H}$ & -6.921934 & 2.169528 & -2.519303 \\
\hline $\mathrm{H}$ & -6.51517 & 1.336381 & -2.795907 \\
\hline $\mathrm{C}$ & -3.537076 & -2.243981 & -1.388764 \\
\hline $\mathrm{H}$ & -3.908348 & -3.185728 & 0.539427 \\
\hline $\mathrm{H}$ & -2.828887 & -3.898523 & -1.347772 \\
\hline $\mathrm{O}$ & -3.733377 & -4.165981 & 1.001444 \\
\hline $\mathrm{H}$ & -5.287359 & -2.888852 & 0.495171 \\
\hline $\mathrm{H}$ & -3.558308 & -1.12312 & 1.037765 \\
\hline $\mathrm{C}$ & -2.773108 & -2.187105 & 2.246466 \\
\hline & -5.75915 & -3.670609 & 0.184584 \\
\hline & & -0.851324 & -3.496062 \\
\hline $\mathrm{H}$ & -0.132218 & & \\
\hline & & & \\
\hline
\end{tabular}




\begin{tabular}{|c|c|c|c|}
\hline $\mathrm{H}$ & -1.161546 & -1.064363 & -3.804063 \\
\hline $\mathrm{H}$ & -0.014557 & 0.220474 & -3.306833 \\
\hline $\mathrm{H}$ & 0.555517 & -1.160694 & -4.288871 \\
\hline $\mathrm{C}$ & 0.414995 & -4.006188 & -1.421098 \\
\hline $\mathrm{H}$ & 0.819548 & -4.189687 & -0.420034 \\
\hline $\mathrm{H}$ & -0.568387 & -4.474958 & -1.514572 \\
\hline $\mathrm{H}$ & 1.094377 & -4.42786 & -2.168406 \\
\hline $\mathrm{O}$ & -1.212625 & -1.310048 & -0.463623 \\
\hline $\mathrm{O}$ & 1.615067 & -0.926098 & -0.463476 \\
\hline $\mathrm{C}$ & 2.729559 & -1.165129 & -1.102211 \\
\hline $\mathrm{C}$ & 3.999678 & -0.633224 & -0.560598 \\
\hline $\mathrm{C}$ & 4.008242 & 0.060992 & 0.655508 \\
\hline $\mathrm{C}$ & 5.186608 & -0.828325 & -1.280434 \\
\hline $\mathrm{C}$ & 6.382936 & -0.32271 & -0.782687 \\
\hline $\mathrm{C}$ & 5.210184 & 0.553664 & 1.15864 \\
\hline $\mathrm{C}$ & 6.398944 & 0.360657 & 0.441455 \\
\hline $\mathrm{H}$ & 3.082594 & 0.211816 & 1.204832 \\
\hline $\mathrm{H}$ & 5.196516 & 1.110906 & 2.093842 \\
\hline $\mathrm{H}$ & 5.16337 & -1.368102 & -2.223956 \\
\hline $\mathrm{H}$ & 7.311652 & -0.456663 & -1.331775 \\
\hline $\mathrm{C}$ & 7.720973 & 0.893136 & 0.925773 \\
\hline $\mathrm{O}$ & 8.624093 & 1.190542 & 0.173182 \\
\hline $\mathrm{O}$ & 7.884847 & 1.036991 & 2.251 \\
\hline $\mathrm{H}$ & 7.136944 & 0.666437 & 2.739997 \\
\hline $\mathrm{O}$ & 2.649375 & -1.842197 & -2.147745 \\
\hline $\mathrm{H}$ & 0.663555 & 0.595758 & -0.245202 \\
\hline $\mathrm{O}$ & -1.03547 & 0.158724 & 1.534426 \\
\hline $\mathrm{H}$ & -0.250194 & 1.948629 & -0.469268 \\
\hline $\mathrm{O}$ & -0.346784 & 3.45125 & -0.352291 \\
\hline $\mathrm{H}$ & -0.556714 & 3.771799 & 0.535341 \\
\hline $\mathrm{H}$ & -1.009774 & 3.849333 & -0.932516 \\
\hline \multicolumn{4}{|c|}{$\begin{array}{l}\text { 34) TS5d: Alcohol Transfer of IM5, G500K }=-1312.59611276 \mathrm{~kJ} / \mathrm{mol} \\
\qquad v=-1039.82 \mathrm{~cm}^{-1}\end{array}$} \\
\hline $\mathrm{Sn}$ & 1.908609 & -1.568334 & -0.699768 \\
\hline $\mathrm{O}$ & 0.492442 & -2.861217 & -0.789156 \\
\hline $\mathrm{C}$ & -0.348373 & -3.396536 & 0.201139 \\
\hline $\mathrm{C}$ & -1.778199 & -2.821767 & 0.331373 \\
\hline $\mathrm{C}$ & -0.131219 & -3.091018 & 1.705688 \\
\hline $\mathrm{H}$ & -0.391013 & -4.482962 & 0.044993 \\
\hline
\end{tabular}




\begin{tabular}{|c|c|c|c|}
\hline $\mathrm{O}$ & 0.797654 & 0.15168 & -0.810582 \\
\hline $\mathrm{C}$ & 0.071536 & 0.720373 & 0.362989 \\
\hline $\mathrm{C}$ & -1.381165 & 0.955391 & 0.03081 \\
\hline $\mathrm{C}$ & -2.265438 & 1.157603 & 1.094169 \\
\hline C & -1.86226 & 0.944064 & -1.284107 \\
\hline C & -3.219048 & 1.13236 & -1.530803 \\
\hline $\mathrm{C}$ & -3.621595 & 1.361169 & 0.848443 \\
\hline $\mathrm{C}$ & -4.105507 & 1.352506 & -0.467652 \\
\hline $\mathrm{H}$ & -1.887543 & 1.133502 & 2.112808 \\
\hline $\mathrm{H}$ & -4.292835 & 1.484302 & 1.697515 \\
\hline $\mathrm{H}$ & -1.178527 & 0.774572 & -2.110961 \\
\hline $\mathrm{H}$ & -3.602291 & 1.113578 & -2.548391 \\
\hline $\mathrm{C}$ & -5.556393 & 1.547903 & -0.789711 \\
\hline $\mathrm{O}$ & -6.078827 & 1.128326 & -1.802759 \\
\hline $\mathrm{O}$ & -6.301663 & 2.229434 & 0.100473 \\
\hline $\mathrm{H}$ & -5.747075 & 2.598094 & 0.80221 \\
\hline $\mathrm{H}$ & -1.76945 & -1.740931 & 0.143178 \\
\hline $\mathrm{C}$ & -1.663998 & -3.088067 & 1.85234 \\
\hline $\mathrm{H}$ & -2.5883 & -3.298902 & -0.22968 \\
\hline $\mathrm{H}$ & -2.044413 & -4.086598 & 2.109973 \\
\hline $\mathrm{O}$ & -2.158956 & -2.120429 & 2.7574 \\
\hline $\mathrm{H}$ & 0.230284 & -2.063332 & 1.84814 \\
\hline $\mathrm{H}$ & 0.459915 & -3.789586 & 2.306149 \\
\hline $\mathrm{H}$ & -3.122713 & -2.151643 & 2.732975 \\
\hline $\mathrm{C}$ & 2.768242 & -1.517624 & -2.621427 \\
\hline $\mathrm{H}$ & 1.979438 & -1.639175 & -3.371429 \\
\hline $\mathrm{H}$ & 3.269016 & -0.555077 & -2.774363 \\
\hline $\mathrm{H}$ & 3.501737 & -2.323612 & -2.729363 \\
\hline $\mathrm{C}$ & 3.060936 & -1.626505 & 1.069417 \\
\hline $\mathrm{H}$ & 4.029703 & -1.14895 & 0.887739 \\
\hline $\mathrm{H}$ & 2.527473 & -1.097416 & 1.863724 \\
\hline $\mathrm{H}$ & 3.22047 & -2.672275 & 1.354263 \\
\hline $\mathrm{O}$ & 0.44647 & 0.371471 & 1.479503 \\
\hline $\mathrm{C}$ & 1.764093 & 2.736587 & 0.699484 \\
\hline $\mathrm{C}$ & 2.314821 & 4.157459 & 0.442915 \\
\hline $\mathrm{C}$ & 3.216192 & 2.194304 & 0.621359 \\
\hline $\mathrm{H}$ & 1.370584 & 2.632471 & 1.721213 \\
\hline $\mathrm{H}$ & 2.325093 & 4.368585 & -0.633644 \\
\hline $\mathrm{C}$ & 3.703015 & 3.629532 & 0.887828 \\
\hline
\end{tabular}




\begin{tabular}{|c|c|c|c|}
\hline $\mathrm{H}$ & 1.870873 & 4.995872 & 0.989623 \\
\hline $\mathrm{H}$ & 3.871546 & 3.803145 & 1.959491 \\
\hline $\mathrm{O}$ & 4.845269 & 4.003399 & 0.139443 \\
\hline $\mathrm{H}$ & 3.462994 & 1.911578 & -0.411669 \\
\hline $\mathrm{H}$ & 3.518223 & 1.402172 & 1.313464 \\
\hline $\mathrm{H}$ & 5.10475 & 4.892993 & 0.406965 \\
\hline $\mathrm{O}$ & 0.810016 & 2.261467 & -0.206413 \\
\hline $\mathrm{H}$ & 1.171621 & 1.181028 & -0.936652 \\
\hline \multicolumn{4}{|c|}{$\begin{array}{l}\text { 35) TS5d+: Alcohol Transfer of IM5- } \mathrm{H}_{3} \mathrm{O}^{+}, \mathrm{G}_{500 \mathrm{~K}}=-1178.91731268 \mathrm{~kJ} / \mathrm{mol} \\
\qquad v=-1685.22 \mathrm{~cm}^{-1}\end{array}$} \\
\hline $\mathrm{Sn}$ & 1.619891 & -1.915897 & -0.946756 \\
\hline $\mathrm{O}$ & -0.249784 & -2.330839 & -0.882117 \\
\hline $\mathrm{C}$ & -0.91052 & -3.076914 & 0.115873 \\
\hline $\mathrm{C}$ & -2.413686 & -2.772127 & 0.27984 \\
\hline $\mathrm{C}$ & -0.709492 & -2.720058 & 1.61333 \\
\hline $\mathrm{H}$ & -0.74093 & -4.150309 & -0.048643 \\
\hline $\mathrm{O}$ & 1.547066 & 0.08636 & -0.408343 \\
\hline $\mathrm{C}$ & 0.759837 & 0.88261 & 0.452447 \\
\hline $\mathrm{C}$ & -0.724456 & 0.88602 & 0.176124 \\
\hline $\mathrm{C}$ & -1.638264 & 0.905334 & 1.227903 \\
\hline $\mathrm{C}$ & -1.171654 & 0.89196 & -1.151286 \\
\hline $\mathrm{C}$ & -2.532708 & 0.90967 & -1.422685 \\
\hline $\mathrm{C}$ & -3.006925 & 0.937442 & 0.955368 \\
\hline $\mathrm{C}$ & -3.458064 & 0.944267 & -0.367967 \\
\hline $\mathrm{H}$ & -1.290448 & 0.8761 & 2.255902 \\
\hline $\mathrm{H}$ & -3.701903 & 0.909945 & 1.79254 \\
\hline $\mathrm{H}$ & -0.456218 & 0.874922 & -1.969661 \\
\hline $\mathrm{H}$ & -2.891474 & 0.898907 & -2.448765 \\
\hline $\mathrm{C}$ & -4.918567 & 0.945579 & -0.714909 \\
\hline $\mathrm{O}$ & -5.354788 & 0.485573 & -1.74969 \\
\hline $\mathrm{O}$ & -5.765853 & 1.487495 & 0.177715 \\
\hline $\mathrm{H}$ & -5.282818 & 1.910228 & 0.901563 \\
\hline $\mathrm{H}$ & -2.599965 & -1.716501 & 0.050715 \\
\hline $\mathrm{C}$ & -2.218445 & -2.954903 & 1.805628 \\
\hline $\mathrm{H}$ & -3.135088 & -3.410516 & -0.23956 \\
\hline $\mathrm{H}$ & -2.422655 & -3.99034 & 2.111287 \\
\hline $\mathrm{O}$ & -2.838286 & -2.045017 & 2.692668 \\
\hline $\mathrm{H}$ & -0.48861 & -1.650805 & 1.728721 \\
\hline $\mathrm{H}$ & -0.013404 & -3.319079 & 2.209218 \\
\hline
\end{tabular}




\begin{tabular}{|c|c|c|c|}
\hline $\mathrm{H}$ & -3.769263 & -2.285274 & 2.768576 \\
\hline $\mathrm{C}$ & 2.200342 & -1.667383 & -2.949771 \\
\hline $\mathrm{H}$ & 1.399217 & -1.143459 & -3.481915 \\
\hline $\mathrm{H}$ & 3.123325 & -1.080682 & -3.000564 \\
\hline $\mathrm{H}$ & 2.366541 & -2.64544 & -3.412735 \\
\hline $\mathrm{C}$ & 2.717536 & -2.836046 & 0.598441 \\
\hline $\mathrm{H}$ & 3.748277 & -2.468331 & 0.593916 \\
\hline $\mathrm{H}$ & 2.243539 & -2.606159 & 1.558125 \\
\hline $\mathrm{H}$ & 2.713947 & -3.920846 & 0.447946 \\
\hline $\mathrm{C}$ & 1.761808 & 3.246573 & 0.634702 \\
\hline $\mathrm{C}$ & 2.219757 & 4.466187 & -0.175147 \\
\hline $\mathrm{C}$ & 3.127172 & 3.208242 & 1.344288 \\
\hline $\mathrm{H}$ & 0.923937 & 3.45122 & 1.306519 \\
\hline $\mathrm{H}$ & 2.675811 & 4.149604 & -1.120292 \\
\hline $\mathrm{C}$ & 3.325462 & 4.671376 & 0.898051 \\
\hline $\mathrm{H}$ & 1.493139 & 5.263924 & -0.349071 \\
\hline $\mathrm{H}$ & 3.004371 & 5.377736 & 1.673997 \\
\hline $\mathrm{O}$ & 4.634334 & 4.958901 & 0.461885 \\
\hline $\mathrm{H}$ & 3.827612 & 2.561972 & 0.801342 \\
\hline $\mathrm{H}$ & 3.141287 & 2.986469 & 2.414931 \\
\hline $\mathrm{H}$ & 4.703462 & 5.908347 & 0.306264 \\
\hline $\mathrm{O}$ & 1.367117 & 2.126006 & -0.177061 \\
\hline $\mathrm{H}$ & 1.997561 & 1.2187 & -0.682013 \\
\hline $\mathrm{O}$ & 1.043888 & 0.751898 & 1.752135 \\
\hline $\mathrm{H}$ & 2.002342 & 0.520633 & 1.892234 \\
\hline $\mathrm{O}$ & 3.575718 & 0.035591 & 2.200267 \\
\hline $\mathrm{H}$ & 3.642383 & -0.736209 & 2.777172 \\
\hline $\mathrm{H}$ & 4.163323 & 0.68998 & 2.599012 \\
\hline
\end{tabular}

36) TS6a: Hydroxyl Lewis-acid reaction with IM6, G500K $=-2350.14024935 \mathrm{~kJ} / \mathrm{mol}$

\begin{tabular}{|l|r|r|r|}
\hline \multicolumn{4}{|c|}{$v=-335.87 \mathrm{~cm}^{-1}$} \\
\hline $\mathrm{Sn}$ & -1.412619 & -1.375186 & 0.069138 \\
\hline $\mathrm{O}$ & -2.189007 & 0.637075 & 2.574223 \\
\hline $\mathrm{O}$ & -3.051675 & -0.198095 & 0.67204 \\
\hline $\mathrm{C}$ & -3.082391 & 0.559362 & 1.728533 \\
\hline $\mathrm{C}$ & -4.347224 & 1.380518 & 1.866403 \\
\hline $\mathrm{C}$ & -4.480971 & 2.262121 & 2.946257 \\
\hline $\mathrm{C}$ & -5.380955 & 1.270983 & 0.926497 \\
\hline $\mathrm{C}$ & -6.537752 & 2.033909 & 1.06672 \\
\hline
\end{tabular}




\begin{tabular}{|c|c|c|c|}
\hline $\mathrm{C}$ & -6.668111 & 2.916871 & 2.147357 \\
\hline $\mathrm{H}$ & -3.673057 & 2.339651 & 3.670254 \\
\hline $\mathrm{H}$ & -5.732117 & 3.715879 & 3.927177 \\
\hline $\mathrm{H}$ & -5.26974 & 0.585773 & 0.090399 \\
\hline $\mathrm{H}$ & -7.344487 & 1.955444 & 0.341315 \\
\hline $\mathrm{C}$ & -7.922481 & 3.719129 & 2.257614 \\
\hline $\mathrm{O}$ & -8.850384 & 3.659979 & 1.470493 \\
\hline $\mathrm{O}$ & -7.940576 & 4.532586 & 3.32621 \\
\hline $\mathrm{H}$ & -8.785605 & 5.009067 & 3.313832 \\
\hline $\mathrm{C}$ & -1.151391 & -2.396001 & 1.905357 \\
\hline $\mathrm{H}$ & -0.637555 & -3.355216 & 1.777216 \\
\hline $\mathrm{H}$ & -0.589504 & -1.761479 & 2.598536 \\
\hline $\mathrm{H}$ & -2.138297 & -2.579111 & 2.34481 \\
\hline $\mathrm{C}$ & -2.470787 & -2.032015 & -1.642906 \\
\hline $\mathrm{H}$ & -2.136479 & -1.453397 & -2.511605 \\
\hline $\mathrm{H}$ & -2.272946 & -3.094259 & -1.825477 \\
\hline $\mathrm{H}$ & -3.544944 & -1.878888 & -1.497948 \\
\hline $\mathrm{O}$ & -0.41364 & 0.359316 & 0.005039 \\
\hline $\mathrm{C}$ & 0.190699 & 0.856834 & -1.041102 \\
\hline $\mathrm{O}$ & 0.153937 & 0.372756 & -2.170454 \\
\hline $\mathrm{C}$ & 0.962224 & 2.110957 & -0.729891 \\
\hline $\mathrm{C}$ & 1.870216 & 2.613493 & -1.667705 \\
\hline $\mathrm{C}$ & 0.796852 & 2.754327 & 0.503122 \\
\hline $\mathrm{C}$ & 2.635851 & 3.737641 & -1.366596 \\
\hline $\mathrm{H}$ & 1.98803 & 2.104135 & -2.621159 \\
\hline $\mathrm{C}$ & 1.543732 & 3.891012 & 0.798137 \\
\hline $\mathrm{H}$ & 0.09091 & 2.352527 & 1.224863 \\
\hline $\mathrm{C}$ & 2.476931 & 4.374726 & -0.127645 \\
\hline $\mathrm{H}$ & 3.358829 & 4.112296 & -2.085729 \\
\hline $\mathrm{H}$ & 1.426341 & 4.397945 & 1.752709 \\
\hline $\mathrm{C}$ & 3.290725 & 5.56741 & 0.252967 \\
\hline $\mathrm{O}$ & 3.168106 & 6.177161 & 1.300085 \\
\hline $\mathrm{O}$ & 4.190733 & 5.915545 & -0.679988 \\
\hline $\mathrm{H}$ & 4.670372 & 6.690222 & -0.34662 \\
\hline $\mathrm{C}$ & 2.382704 & -2.320659 & -0.69171 \\
\hline $\mathrm{C}$ & 2.748984 & -1.13258 & 0.121486 \\
\hline $\mathrm{C}$ & 2.266427 & -0.915402 & 1.416048 \\
\hline $\mathrm{C}$ & 3.645103 & -0.233381 & -0.467395 \\
\hline $\mathrm{C}$ & 2.674128 & 0.210708 & 2.121019 \\
\hline
\end{tabular}




\begin{tabular}{|c|c|c|c|}
\hline $\mathrm{H}$ & 1.578403 & -1.618679 & 1.874283 \\
\hline $\mathrm{C}$ & 4.066591 & 0.884071 & 0.24851 \\
\hline $\mathrm{H}$ & 4.011014 & -0.413864 & -1.474546 \\
\hline $\mathrm{C}$ & 3.581448 & 1.108785 & 1.539709 \\
\hline $\mathrm{H}$ & 2.290462 & 0.389027 & 3.12114 \\
\hline $\mathrm{H}$ & 4.766886 & 1.588862 & -0.191454 \\
\hline $\mathrm{C}$ & 4.062751 & 2.324367 & 2.265725 \\
\hline $\mathrm{O}$ & 4.941147 & 3.060288 & 1.856663 \\
\hline $\mathrm{O}$ & 3.427749 & 2.530891 & 3.427821 \\
\hline $\mathrm{H}$ & 3.796661 & 3.33648 & 3.823719 \\
\hline $\mathrm{O}$ & 2.774194 & -2.656098 & -1.762308 \\
\hline $\mathrm{O}$ & 2.060455 & -3.570982 & 0.346503 \\
\hline $\mathrm{C}$ & 2.718542 & -4.833153 & 0.042918 \\
\hline $\mathrm{C}$ & 2.220013 & -5.645429 & -1.163663 \\
\hline $\mathrm{C}$ & 2.331972 & -5.984913 & 0.98035 \\
\hline $\mathrm{H}$ & 3.785622 & -4.606924 & 0.025584 \\
\hline $\mathrm{H}$ & 1.164589 & -5.43096 & -1.374102 \\
\hline $\mathrm{C}$ & 2.326132 & -6.902671 & -0.275982 \\
\hline $\mathrm{H}$ & 2.800649 & -5.598265 & -2.08714 \\
\hline $\mathrm{H}$ & 3.290986 & -7.408863 & -0.405672 \\
\hline $\mathrm{O}$ & 1.255119 & -7.803762 & -0.435845 \\
\hline $\mathrm{H}$ & 1.307663 & -5.859341 & 1.351541 \\
\hline $\mathrm{H}$ & 3.009441 & -6.216323 & 1.805956 \\
\hline $\mathrm{H}$ & 1.459596 & -8.612779 & 0.047751 \\
\hline $\mathrm{H}$ & 1.094166 & -3.597092 & 0.119248 \\
\hline $\mathrm{O}$ & 0.419121 & -2.275081 & -0.752662 \\
\hline $\mathrm{H}$ & 0.335423 & -2.27687 & -1.716888 \\
\hline
\end{tabular}

37) TS6b: Carbonyl Lewis-acid reaction with IM6, G500K $=-2350.91428935 \mathrm{~kJ} / \mathrm{mol}$

\begin{tabular}{|l|r|r|r|}
\hline \multicolumn{4}{|c|}{$v=-1363.73 \mathrm{~cm}^{-1}$} \\
\hline $\mathrm{Sn}$ & -2.114257 & -3.473288 & -0.390514 \\
\hline $\mathrm{O}$ & -4.89378 & -3.356415 & -0.969974 \\
\hline $\mathrm{O}$ & -3.433955 & -1.841457 & -0.248235 \\
\hline $\mathrm{C}$ & -4.627606 & -2.200038 & -0.610915 \\
\hline $\mathrm{C}$ & -5.681626 & -1.127546 & -0.567817 \\
\hline $\mathrm{C}$ & -7.009409 & -1.457434 & -0.866505 \\
\hline $\mathrm{C}$ & -5.345217 & 0.191037 & -0.232777 \\
\hline $\mathrm{C}$ & -6.332287 & 1.172859 & -0.197521 \\
\hline $\mathrm{C}$ & -8.000112 & -0.478731 & -0.827508 \\
\hline
\end{tabular}




\begin{tabular}{|c|c|c|c|}
\hline $\mathrm{H}$ & -7.256033 & -2.484655 & -1.125122 \\
\hline $\mathrm{H}$ & -9.030878 & -0.736875 & -1.055184 \\
\hline $\mathrm{H}$ & -4.311604 & 0.437428 & -0.00398 \\
\hline $\mathrm{H}$ & -6.083837 & 2.200473 & 0.058051 \\
\hline C & -8.689331 & 1.923825 & -0.43591 \\
\hline $\mathrm{O}$ & -8.45084 & 3.084612 & -0.155449 \\
\hline $\mathrm{O}$ & -9.927801 & 1.495324 & -0.727406 \\
\hline $\mathrm{H}$ & -10.522571 & 2.259354 & -0.665156 \\
\hline $\mathrm{C}$ & -2.836213 & -4.632173 & 1.220148 \\
\hline $\mathrm{H}$ & -3.808061 & -4.244446 & 1.54083 \\
\hline $\mathrm{H}$ & -2.948012 & -5.683143 & 0.931979 \\
\hline $\mathrm{H}$ & -2.12363 & -4.564936 & 2.050196 \\
\hline $\mathrm{C}$ & -2.065897 & -3.672014 & -2.487373 \\
\hline $\mathrm{H}$ & -2.679136 & -2.886429 & -2.939587 \\
\hline $\mathrm{H}$ & -1.031268 & -3.584684 & -2.830233 \\
\hline $\mathrm{H}$ & -2.465914 & -4.652771 & -2.767328 \\
\hline $\mathrm{O}$ & -0.80793 & -2.249097 & 0.531811 \\
\hline $\mathrm{C}$ & -0.044645 & -1.410926 & -0.119859 \\
\hline $\mathrm{O}$ & -0.051849 & -1.277254 & -1.341351 \\
\hline $\mathrm{C}$ & 0.873256 & -0.608917 & 0.76544 \\
\hline $\mathrm{C}$ & 1.824739 & 0.234939 & 0.180501 \\
\hline $\mathrm{C}$ & 0.784911 & -0.703085 & 2.161204 \\
\hline $\mathrm{C}$ & 2.686928 & 0.980331 & 0.982042 \\
\hline $\mathrm{H}$ & 1.883109 & 0.301168 & -0.903375 \\
\hline $\mathrm{C}$ & 1.642954 & 0.042291 & 2.965504 \\
\hline $\mathrm{H}$ & 0.041349 & -1.358171 & 2.606844 \\
\hline $\mathrm{C}$ & 2.597326 & 0.883731 & 2.37858 \\
\hline $\mathrm{H}$ & 3.425483 & 1.634535 & 0.5266 \\
\hline $\mathrm{H}$ & 1.581112 & -0.023078 & 4.049415 \\
\hline $\mathrm{C}$ & 3.499227 & 1.665567 & 3.277577 \\
\hline $\mathrm{O}$ & 3.46913 & 1.615207 & 4.493741 \\
\hline $\mathrm{O}$ & 4.365145 & 2.444961 & 2.610916 \\
\hline $\mathrm{H}$ & 4.90434 & 2.911302 & 3.269125 \\
\hline $\mathrm{C}$ & 0.284347 & -5.757963 & 0.202339 \\
\hline $\mathrm{C}$ & 0.532611 & -5.508304 & 1.671529 \\
\hline $\mathrm{C}$ & 0.174081 & -6.423371 & 2.663925 \\
\hline $\mathrm{C}$ & 1.145991 & -4.297906 & 2.016482 \\
\hline $\mathrm{C}$ & 0.427123 & -6.129576 & 4.003043 \\
\hline $\mathrm{H}$ & -0.307515 & -7.359317 & 2.398803 \\
\hline
\end{tabular}




\begin{tabular}{|c|c|c|c|}
\hline $\mathrm{C}$ & 1.41696 & -4.013285 & 3.349837 \\
\hline $\mathrm{H}$ & 1.407594 & -3.58067 & 1.243722 \\
\hline $\mathrm{C}$ & 1.055861 & -4.926913 & 4.348358 \\
\hline $\mathrm{H}$ & 0.138187 & -6.838422 & 4.773773 \\
\hline $\mathrm{H}$ & 1.903729 & -3.080916 & 3.625635 \\
\hline $\mathrm{C}$ & 1.355541 & -4.573437 & 5.769103 \\
\hline $\mathrm{O}$ & 1.896789 & -3.54042 & 6.117595 \\
\hline $\mathrm{O}$ & 0.966291 & -5.514715 & 6.642191 \\
\hline $\mathrm{H}$ & 1.196973 & -5.201787 & 7.53128 \\
\hline $\mathrm{O}$ & -0.264663 & -4.885028 & -0.518612 \\
\hline $\mathrm{O}$ & 1.547415 & -6.361987 & -0.486862 \\
\hline $\mathrm{C}$ & 2.854714 & -6.298389 & 0.099882 \\
\hline $\mathrm{C}$ & 3.930859 & -7.02492 & -0.721253 \\
\hline $\mathrm{C}$ & 3.192282 & -7.189619 & 1.313343 \\
\hline $\mathrm{H}$ & 3.087447 & -5.242731 & 0.264285 \\
\hline $\mathrm{H}$ & 3.491952 & -7.880503 & -1.248488 \\
\hline $\mathrm{C}$ & 4.542344 & -7.498677 & 0.612808 \\
\hline $\mathrm{H}$ & 4.54537 & -6.432043 & -1.403304 \\
\hline $\mathrm{H}$ & 5.334914 & -6.821059 & 0.956084 \\
\hline $\mathrm{O}$ & 4.996165 & -8.834399 & 0.589631 \\
\hline $\mathrm{H}$ & 2.565161 & -8.089364 & 1.328591 \\
\hline $\mathrm{H}$ & 3.209017 & -6.723304 & 2.302344 \\
\hline $\mathrm{H}$ & 5.450811 & -9.016939 & 1.420458 \\
\hline $\mathrm{H}$ & 0.758929 & -7.315741 & -0.425981 \\
\hline $\mathrm{O}$ & -0.341133 & -7.187103 & 0.035667 \\
\hline $\mathrm{H}$ & -1.007302 & -7.108975 & -0.668362 \\
\hline \multicolumn{4}{|c|}{$\begin{array}{c}\text { 38) TS6: Alcohol Transfer of IM6, G500K }=-1650.86832895 \mathrm{~kJ} / \mathrm{mol} \\
v=-929.15 \mathrm{~cm}^{-1}\end{array}$} \\
\hline $\mathrm{Sn}$ & 0.529489 & -2.095254 & -0.947225 \\
\hline $\mathrm{O}$ & -1.465836 & -1.59759 & -0.726751 \\
\hline $\mathrm{O}$ & 0.787238 & -0.038395 & -1.025714 \\
\hline $\mathrm{C}$ & 0.339604 & 0.836403 & 0.07582 \\
\hline $\mathrm{C}$ & -0.667418 & 1.850958 & -0.400542 \\
\hline $\mathrm{C}$ & -1.376593 & 2.571267 & 0.565592 \\
\hline $\mathrm{C}$ & -0.92127 & 2.072202 & -1.759408 \\
\hline $\mathrm{C}$ & -1.876456 & 3.007196 & -2.147038 \\
\hline $\mathrm{C}$ & -2.323985 & 3.517476 & 0.179129 \\
\hline $\mathrm{C}$ & -2.577352 & 3.743397 & -1.181433 \\
\hline $\mathrm{H}$ & -1.190191 & 2.378613 & 1.619222 \\
\hline
\end{tabular}




\begin{tabular}{|c|c|c|c|}
\hline $\mathrm{H}$ & -2.879728 & 4.04168 & 0.955879 \\
\hline $\mathrm{H}$ & -0.376605 & 1.507841 & -2.511052 \\
\hline $\mathrm{H}$ & -2.083152 & 3.178348 & -3.200911 \\
\hline $\mathrm{C}$ & -3.595772 & 4.734448 & -1.659003 \\
\hline $\mathrm{O}$ & -4.120717 & 4.678536 & -2.753048 \\
\hline $\mathrm{O}$ & -3.931802 & 5.733088 & -0.821038 \\
\hline $\mathrm{H}$ & -3.375542 & 5.721007 & -0.029858 \\
\hline $\mathrm{C}$ & 1.04836 & -2.484613 & -2.947415 \\
\hline $\mathrm{H}$ & 0.241061 & -2.126351 & -3.595273 \\
\hline $\mathrm{H}$ & 1.974083 & -1.955538 & -3.196643 \\
\hline $\mathrm{H}$ & 1.186743 & -3.55983 & -3.096083 \\
\hline $\mathrm{C}$ & 1.454522 & -2.734802 & 0.826525 \\
\hline $\mathrm{H}$ & 2.444818 & -2.274936 & 0.903423 \\
\hline $\mathrm{H}$ & 0.840239 & -2.410446 & 1.671827 \\
\hline $\mathrm{H}$ & 1.547896 & -3.825216 & 0.820284 \\
\hline $\mathrm{O}$ & 0.329257 & 0.369134 & 1.209481 \\
\hline $\mathrm{C}$ & 2.809056 & 1.706133 & 0.734124 \\
\hline $\mathrm{C}$ & 4.033047 & 2.649045 & 0.635356 \\
\hline $\mathrm{C}$ & 3.787424 & 0.508922 & 0.883135 \\
\hline $\mathrm{H}$ & 2.258753 & 1.862565 & 1.675629 \\
\hline $\mathrm{H}$ & 4.318959 & 2.782803 & -0.415451 \\
\hline $\mathrm{C}$ & 4.879747 & 1.519105 & 1.273819 \\
\hline $\mathrm{H}$ & 3.987025 & 3.619332 & 1.141508 \\
\hline $\mathrm{H}$ & 4.946831 & 1.637213 & 2.364273 \\
\hline $\mathrm{O}$ & 6.156874 & 1.234815 & 0.729562 \\
\hline $\mathrm{H}$ & 4.012992 & 0.07563 & -0.100851 \\
\hline $\mathrm{H}$ & 3.537025 & -0.282797 & 1.596572 \\
\hline $\mathrm{H}$ & 6.776959 & 1.899628 & 1.05177 \\
\hline $\mathrm{O}$ & 1.913292 & 1.727032 & -0.331565 \\
\hline $\mathrm{H}$ & 1.691059 & 0.563109 & -1.041863 \\
\hline $\mathrm{C}$ & -1.947793 & -2.798314 & -0.747703 \\
\hline $\mathrm{C}$ & -3.413207 & -2.989644 & -0.623808 \\
\hline $\mathrm{O}$ & -1.136859 & -3.748472 & -0.875514 \\
\hline $\mathrm{C}$ & -3.942184 & -4.285311 & -0.621689 \\
\hline $\mathrm{C}$ & -4.255726 & -1.873973 & -0.518089 \\
\hline $\mathrm{C}$ & -5.317695 & -4.468511 & -0.497894 \\
\hline $\mathrm{H}$ & -3.277596 & -5.140213 & -0.718738 \\
\hline $\mathrm{C}$ & -5.630456 & -2.058624 & -0.409022 \\
\hline $\mathrm{H}$ & -3.830671 & -0.87344 & -0.524452 \\
\hline
\end{tabular}




\begin{tabular}{|l|r|r|r|}
\hline $\mathrm{C}$ & -6.1629 & -3.355089 & -0.386812 \\
\hline $\mathrm{H}$ & -5.718039 & -5.480771 & -0.524222 \\
\hline $\mathrm{H}$ & -6.299532 & -1.204803 & -0.33464 \\
\hline $\mathrm{C}$ & -7.655332 & -3.496791 & -0.267333 \\
\hline $\mathrm{O}$ & -8.432914 & -2.656623 & -0.668024 \\
\hline $\mathrm{O}$ & -8.124995 & -4.613213 & 0.315262 \\
\hline $\mathrm{H}$ & -7.404895 & -5.140666 & 0.688611 \\
\hline
\end{tabular}

39) TS6+: Alcohol Transfer of IM6- $\mathrm{H}_{3} \mathrm{O}^{+}$to $\mathrm{O}_{\text {carbonyl, }} \mathrm{G}_{500 \mathrm{~K}}=-1514.16868087 \mathrm{~kJ} / \mathrm{mol}$

\begin{tabular}{|l|r|r|r|}
\hline \multicolumn{4}{|c|}{$v=-1507.06 \mathrm{~cm}^{-1}$} \\
\hline $\mathrm{Sn}$ & 0.534164 & -2.456581 & -0.906498 \\
\hline $\mathrm{O}$ & -1.289421 & -1.640054 & -0.404218 \\
\hline $\mathrm{O}$ & 1.373968 & -0.612751 & -0.297453 \\
\hline $\mathrm{C}$ & 0.96931 & 0.527584 & 0.424802 \\
\hline $\mathrm{C}$ & -0.33888 & 1.15346 & -0.001084 \\
\hline $\mathrm{C}$ & -1.296801 & 1.500872 & 0.950216 \\
\hline $\mathrm{C}$ & -0.592838 & 1.35001 & -1.363613 \\
\hline $\mathrm{C}$ & -1.815035 & 1.866749 & -1.774189 \\
\hline $\mathrm{C}$ & -2.516781 & 2.042597 & 0.540232 \\
\hline $\mathrm{H}$ & -2.782164 & 2.221133 & -0.82213 \\
\hline $\mathrm{H}$ & -1.104142 & 1.333431 & 2.005438 \\
\hline $\mathrm{H}$ & -3.263606 & 2.275578 & 1.297709 \\
\hline $\mathrm{H}$ & 0.158141 & 1.088677 & -2.105152 \\
\hline $\mathrm{C}$ & -2.028262 & 2.00385 & -2.83122 \\
\hline $\mathrm{O}$ & -4.096856 & 2.748039 & -1.320146 \\
\hline $\mathrm{O}$ & -4.558197 & 2.456855 & -2.403826 \\
\hline $\mathrm{H}$ & -4.778444 & 3.576621 & -0.509797 \\
\hline $\mathrm{C}$ & -4.250111 & 3.816021 & 0.264508 \\
\hline $\mathrm{H}$ & 1.037602 & -2.241727 & -2.933152 \\
\hline $\mathrm{H}$ & 0.308114 & -1.572353 & -3.402133 \\
\hline $\mathrm{H}$ & 2.038831 & -1.807659 & -3.018634 \\
\hline $\mathrm{C}$ & 1.010113 & -3.216368 & -3.429515 \\
\hline $\mathrm{H}$ & 1.268243 & -3.704965 & 0.612482 \\
\hline $\mathrm{H}$ & 2.351538 & -3.818322 & 0.503289 \\
\hline $\mathrm{H}$ & 1.047213 & -3.24627 & 1.581675 \\
\hline $\mathrm{C}$ & 0.786267 & -4.68456 & 0.541743 \\
\hline $\mathrm{C}$ & 2.973386 & 2.144423 & 0.585098 \\
\hline $\mathrm{C}$ & 3.969747 & 2.981901 & -0.227509 \\
\hline & 4.146436 & 1.484906 & 1.33496 \\
\hline $\mathrm{H}$ & 2.322289 & 2.739921 & \\
\hline & & & \\
\hline & & & \\
\hline
\end{tabular}




\begin{tabular}{|l|r|r|r|}
\hline $\mathrm{H}$ & 4.245062 & 2.459912 & -1.151305 \\
\hline $\mathrm{C}$ & 5.015434 & 2.674995 & 0.880698 \\
\hline $\mathrm{H}$ & 3.710228 & 4.022567 & -0.438516 \\
\hline $\mathrm{H}$ & 5.040548 & 3.468502 & 1.638389 \\
\hline $\mathrm{O}$ & 6.318906 & 2.308714 & 0.48823 \\
\hline $\mathrm{H}$ & 4.477136 & 0.574267 & 0.820218 \\
\hline $\mathrm{H}$ & 4.032705 & 1.298192 & 2.405593 \\
\hline $\mathrm{O}$ & 6.82687 & 3.112076 & 0.323969 \\
\hline $\mathrm{H}$ & 2.110524 & 1.321597 & -0.220377 \\
\hline $\mathrm{C}$ & 2.280971 & 0.191884 & -0.612731 \\
\hline $\mathrm{C}$ & -2.021404 & -2.611713 & -0.847545 \\
\hline $\mathrm{O}$ & -3.494567 & -2.490948 & -0.779335 \\
\hline $\mathrm{C}$ & -1.42452 & -3.607433 & -1.33113 \\
\hline $\mathrm{C}$ & -4.300619 & -3.548056 & -1.21625 \\
\hline $\mathrm{C}$ & -4.066381 & -1.305735 & -0.297128 \\
\hline $\mathrm{H}$ & -5.686965 & -3.424154 & -1.154692 \\
\hline $\mathrm{C}$ & -3.843573 & -4.454985 & -1.604229 \\
\hline $\mathrm{H}$ & -5.450334 & -1.178066 & -0.25203 \\
\hline $\mathrm{C}$ & -3.423585 & -0.492983 & 0.032228 \\
\hline $\mathrm{H}$ & -6.263045 & -2.241744 & -0.66818 \\
\hline $\mathrm{H}$ & -6.302208 & -4.244475 & -1.521224 \\
\hline $\mathrm{C}$ & -5.910078 & -0.261438 & 0.109239 \\
\hline $\mathrm{O}$ & -7.753671 & -2.051338 & -0.593696 \\
\hline $\mathrm{O}$ & -8.281997 & -0.961026 & -0.643973 \\
\hline $\mathrm{H}$ & -8.512032 & -3.152403 & -0.461778 \\
\hline $\mathrm{O}$ & -7.965336 & -3.93961 & -0.330682 \\
\hline $\mathrm{H}$ & 1.083647 & 0.422646 & 1.750365 \\
\hline $\mathrm{O}$ & 1.789204 & -0.232913 & 2.002948 \\
\hline $\mathrm{H}$ & 2.827794 & -1.379052 & 2.631207 \\
\hline & 3.554398 & -1.676289 & 2.069443 \\
\hline $\mathrm{H}$ & 3.241977 & -1.143648 & 3.471226 \\
\hline & & & \\
\hline $\mathrm{H}$ & & & \\
\hline
\end{tabular}




\section{References}

(1) Parshall, G. W.; Ittel, S. D. Homogeneous Catalysis: The Applications and Chemistry of Catalysis by Soluble Transition Metal Complexes, 2nd ed.; Wiley: New York, 1992.

(2) Schuchardt, K. L.; Didier, B. T.; Elsethagen, T.; Sun, L.; Gurumoorthi, V.; Chase, J.; Li, J.; Windus, T. L. Basis Set Exchange: A Community Database for Computational Sciences. J. Chem. Inf. Model. 2007, 47 (3), 1045-1052. https://doi.org/10.1021/ci600510j.

(3) Feller, D. The Role of Databases in Support of Computational Chemistry Calculations. J. Comput. Chem. 1996, 17 (13), 1571-1586. https://doi.org/10.1002/(SICI)1096987X(199610)17:13<1571::AID-JCC9>3.0.CO;2-P.

(4) Gao, C. W.; Allen, J. W.; Green, W. H.; West, R. H. Reaction Mechanism Generator: Automatic Construction of Chemical Kinetic Mechanisms. Comput. Phys. Commun. 2016, 203, 212-225. https://doi.org/10.1016/j.cpc.2016.02.013. 